
\title{
The Phenomenology of Right Handed Neutrinos
}

\author{
Marco Drewes \\ Physik Department T70, Technische Universität München, \\ James Franck Straße 1, D-85748 Garching, Germany
}

\begin{abstract}
Neutrinos are the only matter particles in the Standard Model of particle physics that have only been observed with left handed chirality to date. If right handed neutrinos exist, they could be responsible for several phenomena that have no explanation within the Standard Model, including neutrino oscillations, the baryon asymmetry of the universe, dark matter and dark radiation. After a pedagogical introduction, we review recent progress in the phenomenology of right handed neutrinos. We in particular discuss the mass ranges suggested by hints for neutrino oscillation anomalies and dark radiation $(\mathrm{eV})$, sterile neutrino dark matter scenarios (keV) and experimentally testable theories of baryogenesis ( $\mathrm{GeV}$ to $\mathrm{TeV})$. We summarize constraints from theoretical considerations, laboratory experiments, astrophysics and cosmology for each of these.
\end{abstract}

\section{Contents}

1 Introduction

1.1 Physics beyond the Standard Model . . . . . . . . . . . . . . . . . . . . . . . 2

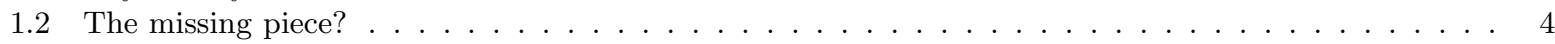

1.3 The range of right handed neutrino masses . . . . . . . . . . . . . . . . . . . 5

2 Neutrino oscillations 6

2.1 The standard scenario of massive neutrinos . . . . . . . . . . . . . . . . . 7

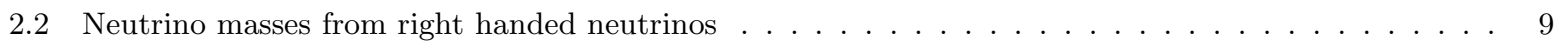

2.3 The seesaw mechanism . . . . . . . . . . . . . . . . . . . . . . . 10

3 Other laboratory experiments 12

3.1 Neutrino oscillation anomalies . . . . . . . . . . . . . . . . . . . . . . . . 12

3.2 Lepton flavour violation . . . . . . . . . . . . . . . . . . . . . . . . . 14

3.3 Neutrinoless double $\beta$-decay . . . . . . . . . . . . . . . . . . . . . . . . . . . . . 14

3.4 Collider searches . . . . . . . . . . . . . . . . . . . . . . . . 17

3.5 Direct dark matter searches . . . . . . . . . . . . . . . . . . . . . . . 18

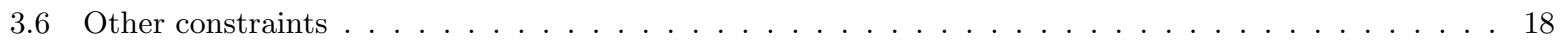

4 Thermal history of the universe 19

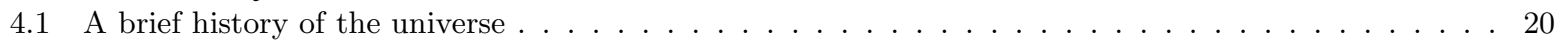

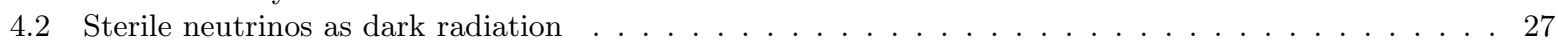

4.3 Sterile neutrinos in astrophysics . . . . . . . . . . . . . . . . . . . . . . . . 29

5 Baryogenesis via leptogenesis $\mathbf{2 9}$

5.1 Leptogenesis from $N_{I}$ freezeout and decay . . . . . . . . . . . . . . . . . . . . . . . . . . . . . . . . . .

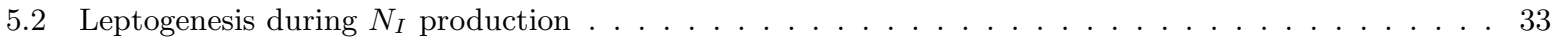

5.3 Towards a quantitative treatment . . . . . . . . . . . . . . . . . . . . . . . . .

6 Sterile neutrinos as dark matter 39

7 A theory of almost everything 44

8 Conclusions 46

A Overview Table: Majorana mass scales and observables 48

B Dirac and Majorana masses 49 


\section{Introduction}

This review is intended to give a comprehensive overview of what we know (more precisely: what I know) about the phenomenology of right handed neutrinos. Faced with the difficulty of writing a text that is readable within reasonable time, and is at the same time is as precise as possible, I provide extra details, explanations and comments for the interested reader in an extensive set of footnotes. For a quick read you may essentially ignore all of them. Furthermore, each section has been written to be as self-contained as possible, so that they can also be read individually as "mini reviews". The basic notations used throughout the review are introduced in sections 1.2 and 2, A very quick summary is given in appendix A.

I will maintain a list of corrections online at http://res-publica.eu/RightHandedNeutrinos.html. If you find any errors or typos I would be very grateful if you could inform me; you can find my most up-to-date contact information on the above website. This preprint is identical to the published article [1] up to a few rephrasings and a number of additional references that appeared after the article had been accepted for publication.

\subsection{Physics beyond the Standard Model}

The Standard Model of particle physics (SM) and theory of General Relativity (GR) form the basic pillars of modern physics. Together they can describe almost all phenomena observed in nature 1 in terms of a small number of underlying principles - general covariance, gauge invariance and quantum mechanics - and a handful of number:2 [2]. All elementary particles we have observed to date can be understood as fundamental excitations of a few quantum fields, the properties of which are constrained by the local structure of space and time 3 . Interactions between them are the result of (gauge) symmetries of the Lagrangian.

In spite of its enormous success, this cannot be a complete theory of nature for two reasons. On one hand, it treats gravitational fields as a classical background, while matter and other interactions are described by quantum field theory in the SM. This approximation certainly becomes invalid and has to be extended to a theory of quantum gravity at energies near the Planck scal $4 M_{P}=1.22 \times 10^{19} \mathrm{GeV}$. We do not address this problem in the following because it is of little relevance for experiments in foreseeable time. On the other hand, there are four experimental and observational facts which cannot be understood in the framework SM+GR. Three of them are widely believed to be related to particle physics,

(I) flavour violation in neutrino experiments, section 2,

(II) the cosmological origin of the baryonic matter in the universe, section 5 ,

\footnotetext{
${ }^{1}$ The basic laws of other areas of natural science and technology can be understood as effective theories, which in principle can be derived from the SM and GR. Though there exist many complex phenomena that we do not understand or cannot predict in detail, this lack of predictivity is almost certainly related to the complexity of the system rather than a lack of understanding of its basic components, the elementary quantum fields.

${ }^{2}$ There are 19 free parameters in the SM; these are usually chosen as six quark masses, three mixing angles and one CP violating phase for the quarks, three charged lepton masses, three gauge couplings, two parameters in the Higgs potential and a QCD vacuum angle. The neutrinos are massless in the SM. GR adds two additional parameters to this barcode of nature, the Planck mass and the cosmological constant.

${ }^{3}$ All known elementary particles transform under irreducible representations of the Poincaré group.

${ }^{4}$ We use natural units $c=\hbar=1$.
} 
(III) the composition and origin of the observed dark matter (DM), section 6 ,

In addition to the above evidence for the existence of "new physics", there are a number of hints in experimental data that may point towards the existence of physics beyond the SM; these have not (yet?) led to a claim of discovery and may also be explained by systematics. Of these, we will only discuss two in detail in this review 5

(i) the statistical preference for additional relativistic particles dubbed "dark radiation" (DR) 6 in some fits to cosmological data, section 4 ,

(ii) the anomalies seen in some short baseline and reactor neutrino experiments, section 3 ,

All of the above phenomena may be related to right handed (RH) neutrinos with different masses. It is the purpose of this article to summarize how they can be connected to these hypothetical particles and review bounds from theoretical considerations, laboratory experiments and cosmology on $\mathrm{RH}$ neutrino properties.

To complete the list, let us add the fourth piece of evidence for physics beyond SM+GR, which is related to gravitation of cosmology,

(IV) the overall geometry of the universe (isotropy, homogeneity and spatial flatness), as e.g. seen in the cosmic microwave background (CMB).

We do not address (IV) here, as it is not related to $\mathrm{RH}$ neutrinos (or particle physics in general) in an obvious way 7 . An intuitive explanation is given by cosmic inflation, see section 4.1. Finally, the observed acceleration of the universe's expansion is often included in this list. However, all observations can currently be explained in terms of a cosmological constant $\Lambda$, which is simply a free parameter in GR. Hence, the accelerated expansion can be accommodated in the framework of SM+GR. The question of the microphysical "origin" of $\Lambda$ (and its smallness) only arises when the SM and GR are interpreted as low energy limits of a more general theory, including a complete description of quantum gravity. To date, (I)-(III) and (IV) are the only confirmed empirical proofs of physics that cannot be explained by $\mathrm{SM}+\mathrm{GR} 8$.

In the remainder of this section we introduce the concept of $\mathrm{RH}$ neutrinos and define our notation. In section 2 we review how they can generate masses for the known neutrinos. In section 3 we summarize bounds from past laboratory experiments on RH neutrino properties, discuss the interpretation of the observed neutrino oscillation anomalies (ii) in terms of RH neutrinos and comment on possible future searches. In sections [4 6] we discuss various cosmological constraints,

\footnotetext{
${ }^{5}$ Others include the long standing issues of the muon magnetic moment (see e.g. 3] for a review), the annual modulation in the DAMA data [4], the excess of positrons in the cosmic radiation [5, [6], the "forward-backward asymmetry" observed at the Tevatron [7], the disputed evidence for neutrinoless double $\beta$-decay claimed by the Heidelberg-Moscow experiment [8], the cosmological lithium problem [9], unexplained features in the galactic $\gamma$ ray spectrum that may be related to DM [10] and shifts in quasar absorption lines that have been interpreted as signatures of a varying fine structure constant [11].

${ }^{6}$ The term "dark radiation" refers to relativistic particles in the early universe with no or tiny interaction with the $\mathrm{SM}$ at temperatures $T<2 \mathrm{MeV}$.

${ }^{7}$ It has been speculated that this point may be related to the RH neutrinos' superpartners [12], see also [13].

${ }^{8}$ There are various aspects of the SM that may be considered "problems" from an aesthetic viewpoint or physical intuition, such as the hierarchy between the electroweak and Planck scale, the strong CP problem, the factorization of the gauge group and the flavour structure. We do not discuss these here. We also do not discuss the issue of vacuum stability, which seems inconclusive at this stage due to uncertainties in the top mass [14 [18].
} 
starting with a general summary in section 4.1. The perspectives to interpret the hints for "dark radiation" (i) in terms of $\mathrm{RH}$ neutrinos and reconcile them with the oscillation anomalies (ii) are addressed in section [4.2. Section 5 is devoted to the idea that RH neutrinos are the origin of the baryonic matter in the universe (leptogenesis) and possible implications for their properties. Section 6 discusses RH neutrinos as DM candidates. In section 7 we address the question how many of these phenomena can be explained simultaneously by $\mathrm{RH}$ neutrinos alone. We conclude in section 8 and give a tabular summary of possible $\mathrm{RH}$ neutrino mass scales and their implications for known and future observations in appendix $\mathrm{A}$.

\subsection{The missing piece?}

All matter we know is composed of elementary fermions with spin $\frac{1}{2}$. These can be described by Weyl spinors, which transform under irreducible representations of the Poncairé group, and combinations thereof. There are two such representations, known as "left chiral" and "right chiral" spinors. Remarkably, all known elementary fermions except neutrinos come in pairs of opposite chirality, i.e. have been observed as "left handed" (LH) and "right handed" (RH) particles 9, see figure 1, For unknown reasons the interactions of the SM are such that both can be combined into a Dirac spinor, see appendix B. Neutrinos, however, so far have only been observed as LH particles. One conclusion that could be derived from this is that no right chiral "partner" for the observed LH neutrinos exists in nature. Another possible conclusion is that we have not seen RH neutrinos just because their interaction with other matter is too weak. Indeed LH neutrinos are electrically and colour neutral; in the SM they only participate in the weak interaction, which does not couple to RH fields. This suggests that their RH partners are singlet under all gauge interaction 10 . Such particles are referred to as "sterile neutrinos".

Let us add $n \mathrm{RH}$ fermions $\nu_{R, i}$ to the SM that are singlet under all gauge interactions and couple to LH neutrinos in same way as RH charged leptons couple to LH charged leptons, i.e. via Yukawa interactions. We will refer to these fields as $R H$ neutrinos and to the index ${ }_{i}$ that labels them as flavour index. Then the most general renormalizable Lagrangian in Minkowski space that only contains SM fields and $\nu_{R}$ reads

$$
\mathcal{L}=\mathcal{L}_{S M}+i \overline{\nu_{R}} \not \nu_{R}-\overline{l_{L}} F \nu_{R} \tilde{\Phi}-\overline{\nu_{R}} F^{\dagger} l_{L} \tilde{\Phi}^{\dagger}-\frac{1}{2}\left(\overline{\nu_{R}^{c}} M_{M} \nu_{R}+\overline{\nu_{R}} M_{M}^{\dagger} \nu_{R}^{c}\right)
$$

Here we have suppressed flavour and isospin indices. $\mathcal{L}_{S M}$ is the Lagrangian of the SM. $F$ is a matrix of Yukawa couplings and $M_{M}$ a Majorana mass term for the right handed neutrinos $\nu_{R}$. $l_{L}=\left(\nu_{L}, e_{L}\right)^{T}$ are the left handed lepton doublets in the SM and $\Phi$ is the Higgs doublet. $\tilde{\Phi}=(\epsilon \Phi)^{\dagger}$, where $\epsilon$ is the $S U(2)$ antisymmetric tensor, and $\nu_{R}^{c}=C{\overline{\nu_{R}}}^{T}$, where the charge conjugation matrix is $C=i \gamma_{2} \gamma_{0}$ in the Weyl representation. We choose a flavour basis where the charged lepton Yukawa couplings and $M_{M}$ are diagonal and the neutrino coupling to weak currents has the form (3) 11 Throughout this article we assume that there are only three "active" neutrinos $\nu_{L}$ that are charged under the weak interaction. If there are more families, then these

\footnotetext{
${ }^{9}$ In this article "RH" and "LH" always refer to the chirality of the fields and not to helicity eigenstates.

${ }^{10}$ Here we refer to the $S U(3) \times S U(2) \times U(1)$ gauge group of the SM. They can of course be charged under some extended gauge group, which either acts only in a "hidden sector" or is broken at energies above the electroweak scale.

${ }^{11}$ This corresponds to a "mass basis" for charged leptons and "flavour basis" for active neutrinos. For vanishing Higgs field value it would coincide with the mass basis for all fields.
} 


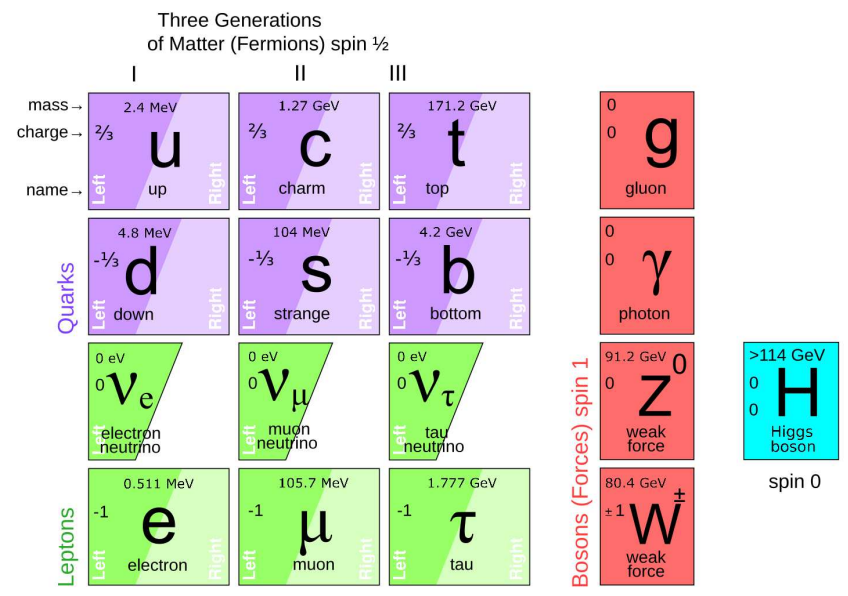

Figure 1: The particle content of the SM. Are we missing the right handed partner of the neutrinos? Picture taken from [21].

must be heavier than $m_{Z} / 2$, otherwise they would contribute to the width of the Z-boson [19], see [20] for some recent discussion and references.

In the Lagrangian (1) the fields $\nu_{R}$ only interact via the Yukawa couplings $F$. In the early universe, when the temperature was high enough that Higgs particles were present in the primordial plasma $\left(T>T_{E W} \sim 140 \mathrm{GeV}\right.$ for a Higgs mass $m_{H} \sim 125 \mathrm{GeV}$ [22, 23]), this interaction allowed $\nu_{R}$-particles to participate in various different scattering processes. At energies much below the mass of the W-boson one can in good approximation replace the Higgs field $\Phi$ by its vacuum expectation value $v=174 \mathrm{GeV}$. Then (1) can be written as

$$
\begin{array}{r}
\mathcal{L}=\mathcal{L}_{S M}+i \overline{\nu_{R}, I}, \partial \nu_{R, I}-\left(m_{D}\right)_{\alpha I} \overline{\nu_{L}, \alpha} \nu_{R, I}-\left(m_{D}\right)_{\alpha I}^{*} \overline{\nu_{R}, I} \nu_{L, \alpha} \\
-\frac{1}{2}\left[\left(M_{M}\right)_{I J} \overline{\nu_{R, I}^{c}} \nu_{R, J}+\left(M_{M}\right)_{I J}^{*} \overline{\nu_{R}, I} \nu_{R, J}^{c}\right]
\end{array}
$$

where we defined the Dirac mass matrix $m_{D}=F v$. Thus, at $T \ll T_{E W}$ the only effect of the Yukawa interaction is the generation of the Dirac mass term $m_{D}$, and the only way how the fields $\nu_{R}$ interact with the SM is via their mixing with $\nu_{L}$ due to $m_{D}$.

\subsection{The range of right handed neutrino masses}

While in the SM the Higgs mass $m_{H}$ is the only dimensionful parameter (apart from the Planck mass), the Lagrangian (1) introduces $n$ new dimensional parameters in $M_{M}$. The scale(s) associated with these provide a convenient way to classify different $\mathrm{RH}$ neutrino scenarios. Various embeddings of (1) into a bigger framework make different predictions for $M_{M}$ (see e.g. [24-26] for a general overview and [27 29] for recent developments in model building), but empirically there are only few constraints. The following scenarios are particularly motivated, a summary in table form is given in appendix $\mathrm{A}$, Of course, $M_{M}$ can have eigenvalues in several different mass ranges, so that several of these scenarios may be combined in nature.

$\boldsymbol{M}_{\boldsymbol{M}} \gtrsim \mathbf{1 0}^{\mathbf{9}} \mathbf{G e V}$ - This range is motivated by embeddings of (1) into GUT scenarios [30], such as $\mathrm{SO}(10)$ unification [31, 32]. $\mathrm{SO}(10)$ models necessarily require the existence of $\nu_{R} 12$ For

\footnotetext{
${ }^{12}$ Any model that contains a $U(1)_{B-L}$ gauge symmetry requires this for anomaly freedom. This can be used as
} 
Yukawa couplings $F$ of order one, RH neutrinos with masses favoured by GUT models reproduce the scale of observed neutrino oscillations (I) via (10). In addition, typical parameter values allow to generate the observed baryon density in the universe (II) in CP-violating decays of RH neutrinos, see section 5.1. The regime $M_{M}<10^{15} \mathrm{GeV}$ is favoured [33].

$M_{M} \sim \mathbf{T e V}$ - Theoretically this mass range is interesting because it follows from a no new scale principle of minimality: If $M_{M}$ is near the electroweak scale, the origin of both scales may be related. The origin of matter (II) may be explained by leptogenesis from CP-violating $\nu_{R^{-}}$ oscillations (see section 5.2) or, if two $\nu_{R}$ masses are degenerate, decays (see section 5.1). Neutrino masses (I) are explained by the seesaw mechanism. From an experimental viewpoint this mass range is favourable because it is accessible by high energy experiments, such as LHC.

$\boldsymbol{M}_{\boldsymbol{M}} \sim \mathrm{GeV}$ - If $M_{M}$ has at least two eigenvalues $\gtrsim 2 \mathrm{GeV}$ and another eigenvalue in the keV range, then the observations (I)-(III) can be described by (1) alone, and no other physics between the electroweak and Planck scales is required, see section 7 . Experimentally $\nu_{R}$ with GeV masses may be found using high intensity experiments, see section 3.4.

$\boldsymbol{M}_{\boldsymbol{M}} \sim \mathbf{k e V}-\mathrm{RH}$ neutrinos with keV masses are promising candidates for the DM (III), see section 6 .

$\boldsymbol{M}_{\boldsymbol{M}} \sim \mathbf{e V}-\mathrm{RH}$ neutrinos with $\mathrm{eV}$ masses can provide an explanation for the anomalies (i) and/or (ii), which are observed in some neutrino experiments (see section 3.1) and cosmological data (see section 4, in particular 4.2).

$\boldsymbol{M}_{\boldsymbol{M}}=\mathbf{0}$ - For $n=3$ the leptonic sector exactly resembles the quark sector without strong interactions. In this case neutrinos are Dirac particles 13 Then neutrino masses are generated by the Higgs mechanism in precisely the same way as other fermion masses, and their smallness can only be assigned to very tiny Yukawa couplings. Though in principle possible, this may appear "unnatural" unless there is a deeper reason for it 14. Furthermore, there is no known principle that forbids $M_{M}$ for the gauge singlet fields $\nu_{R} 15$. This is in contrast to quarks and charged leptons, for which an explicit mass term is forbidden by gauge symmetry.

\section{Neutrino oscillations}

The probably strongest motivation for the existence of $\nu_{R}$ are neutrino oscillations, the only processes amongst (I)-(IV) that have been observed in the laboratory. The neutrinos $\nu_{L}$ are massless in the $\mathrm{SM}^{16}$. In the past two decades an increasing number of neutrino experiments has observed neutrino flavour changes, which indicate that neutrinos are massive and oscillate, see e.g. [27, 38] for reviews with many references. The experimental results can be divided into two categories: the standard 3-scenario (SS) of three massive neutrinos, which we discuss here, and deviations from it, which we discuss in section 3 .

an argument for the existence of $\nu_{R}$ : The conservation of $B-L$ in the SM is not related to a gauge symmetry. If there is such symmetry, then $\nu_{R}$ must exist.

${ }^{13}$ For $n \neq 3$ it is in general not possible to combine all $\nu_{L}$ and $\nu_{R}$ into Dirac spinors, see appendix B]

${ }^{14}$ Some speculations on such reasons can e.g. be found in 34 37].

${ }^{15}$ A small value of $M_{M}$ is, however, "natural" in the technical sense because the symmetry of $\mathcal{L}$ increases in the limit $M_{M} \rightarrow 0$ (there is a global $U(1)_{B-L}$ ).

${ }^{16}$ We do not consider neutrino masses as a part of the SM because we do not know what the nature (Dirac or Majorana) or absolute scale of the mass term is. 
The interactions of neutrinos in the SM are described by the Lagrangian term

$$
-\frac{g}{\sqrt{2}} \overline{\nu_{L}} \gamma^{\mu} e_{L} W_{\mu}^{+}-\frac{g}{\sqrt{2}} \overline{e_{L}} \gamma^{\mu} \nu_{L} W_{\mu}^{-}-\frac{g}{2 \cos \theta_{W}} \overline{\nu_{L}} \gamma^{\mu} \nu_{L} Z_{\mu}
$$

where $g$ is the $S U(2)$ gauge coupling constant and $\theta_{W}$ the weak mixing angle. This defines the basis of weak interaction eigenstates (electron, muon and tau neutrino) 17 If neutrinos have a mass, then the mass term need not be diagonal in this basis in flavour space. In the SS, the weak interaction eigenstates $\nu_{L, e}, \nu_{L, \mu}$ and $\nu_{L, \tau}$ are superpositions of three mass eigenstates $v_{L, i}$ with masses $m_{i}$. For a given momentum, these have different energies if their masses are different, and their wave functions oscillate with different frequencies. Thus, the flavour decomposition of a neutrino state changes in time. This can explain the observed neutrino oscillations.

\subsection{The standard scenario of massive neutrinos}

There are two different ways to effectively realize the SS of three massive neutrinos with no extra particles.

Majorana neutrinos - A Majorana mass term of the form

$$
\frac{1}{2} \overline{\nu_{L}} m_{\nu} \nu_{L}^{c}+\text { h.c. }
$$

can be constructed without adding any new degrees of freedom to the SM. This term, however, breaks gauge invariance unless it is generated by spontaneous symmetry breaking from a gauge invariant term like [39]

$$
\frac{1}{2} \overline{l_{L}} \tilde{\Phi} f \tilde{\Phi}^{T} l_{L}^{c}+h . c .
$$

where $f$ is some flavour matrix of dimension $1 /$ mass. The dimension-5 operator (5) is not renormalizable; in an effective field theory approach it can be understood as the low energy limit of renormalizable operators that is obtained after "integrating out" heavier degrees of freedom. These can, for example (but not necessarily!), be right handed neutrinos, cf. (11). Therefore a Majorana mass term (4) clearly hints towards the existence of new physics although it can be constructed from SM fields only. At low energies one effectively observes the SS with only three massive neutrinos if the energy scale related to the new physics (in case of $\mathrm{RH}$ neutrinos the mass $\left.M_{M}\right)$ is sufficiently high that all new particles are too heavy to be seen in neutrino experiments. The Majorana mass term (4) can be diagonalized by a transformation

$$
m_{\nu}=U_{\nu} \operatorname{diag}\left(m_{1}, m_{2}, m_{3}\right) U_{\nu}^{T} .
$$

Such a transformation is always possible because the most general $m_{\nu}$ is a symmetric matrix. Any antisymmetric part is unphysical due to the simple spinor relation $2 \overline{\nu_{L}} m_{\nu} \nu_{L}^{c}=\overline{\nu_{L}}\left(m_{\nu}+m_{\nu}^{T}\right) \nu_{L}^{c} 18$ The matrix $U_{\nu}$ is constructed from the eigenvectors of $m_{\nu} m_{\nu}^{\dagger}$. In the mass base, the neutrino mixing matrix $U_{\nu}$ appears in the coupling to $W_{\mu}$ in (3).

\footnotetext{
${ }^{17}$ More precisely, if one considers the general form of the interaction $\frac{g}{\sqrt{2}} \overline{\nu_{L, \alpha}} \gamma^{\mu} U_{\alpha \beta} e_{L, \beta} W_{\mu}^{+}$in the basis where charged Yukawa couplings are diagonal, then the basis of weak interaction eigenstates for $\nu_{L}$ is the one where $U_{\alpha \beta}=\delta_{\alpha \beta}$.

${ }^{18}$ For the same reason the Majorana matrix $M_{M}$ in (1) is symmetric in any flavour basis.
} 
Dirac neutrinos - If neutrinos are Dirac particles, the existence of $\nu_{R}$ is directly required to construct the mass term

$$
\overline{\nu_{L}} m_{D} \nu_{R}+h . c . .
$$

Though this means adding new degrees of freedom to the SM at low energies, it is still a realization of the SS, i.e. only three massive neutrinos are observed. The $\nu_{R}$ can be combined with the $\nu_{L}$ into Dirac spinors and there are only three different masses. One could say that there are no new particles in the strict sense, but just additional spin states for neutrinos. We refer to both of these scenarios as the SS.

A bi-unitary transformation $m_{D}=U_{\nu} \operatorname{diag}\left(m_{1}, m_{2}, m_{3}\right) V_{\nu}^{\dagger}$ can diagonalize the mass term (7), with real and positive $m_{i}$. One can define a Dirac spinor $\Psi_{\nu} \equiv V_{\nu}^{\dagger} \nu_{R}+U_{\nu}^{\dagger} \nu_{L}$ with a diagonal mass term $m_{\nu}^{\text {diag }}=\operatorname{diag}\left(m_{1}, m_{2}, m_{3}\right)$, i.e. $\overline{\Psi_{\nu}}\left(i \not \partial-m_{\nu}^{\text {diag }}\right) \Psi_{\nu}$. The matrix $V_{\nu}$ is not physical and can be absorbed into a redefinition of the flavour vector $\nu_{R}$. The neutrino mixing matrix $U_{\nu}$ then appears in the coupling of that Dirac spinor to $W_{\mu}$ in (3). A phenomenological prediction of Dirac neutrinos is that there is no neutrinoless double $\beta$-decay. In order to be consistent with observations, the Yukawa couplings $F$ have to be very small $\left(F \sim 10^{-12}\right)$ compared to those of the charged leptons and quarks.

The neutrino mixing matrix - In the basis where charged Yukawa couplings are diagonal, the mixing matrix $U_{\nu}$ is identical to the Pontecorvo-Maki-Nakagawa-Sakata matrix [40, 41] and can be parametrized as

$$
U_{\nu}=V^{(23)} U_{\delta} V^{(13)} U_{-\delta} V^{(12)} \operatorname{diag}\left(e^{i \alpha_{1} / 2}, e^{i \alpha_{2} / 2}, 1\right)
$$

with $U_{ \pm \delta}=\operatorname{diag}\left(e^{\mp i \delta / 2}, 1, e^{ \pm i \delta / 2}\right)$. The matrices $V^{(i j)}$ are given by

$$
V^{(23)}=\left(\begin{array}{ccc}
1 & 0 & 0 \\
0 & c_{23} & s_{23} \\
0 & -s_{23} & c_{23}
\end{array},\right), V^{(13)}=\left(\begin{array}{ccc}
c_{13} & 0 & s_{13} \\
0 & 1 & 0 \\
-s_{13} & 0 & c_{13}
\end{array}\right), V^{(12)}=\left(\begin{array}{ccc}
c_{12} & s_{12} & 0 \\
-s_{12} & c_{12} & 0 \\
0 & 0 & 1
\end{array}\right),
$$

where $c_{i j}$ and $s_{i j}$ denote $\cos \left(\theta_{i j}\right)$ and $\sin \left(\theta_{i j}\right)$, respectively. $\theta_{i j}$ are the neutrino mixing angles, $\alpha_{1}, \alpha_{2}$ and $\delta$ are $C P$-violating phases. Many parameters of the mixing matrix $U_{\nu}$ have been measured in recent years. In particular, two mass square differences have been determined as $\Delta m_{\text {sol }}^{2} \equiv m_{2}^{2}-m_{1}^{2} \simeq 7.5 \times 10^{-5} \mathrm{eV}^{2}$ and $\Delta m_{\text {atm }}^{2} \equiv\left|m_{3}^{2}-m_{1}^{2}\right| \simeq 2.4 \times 10^{-3} \mathrm{eV}^{2}$, the mixing angles are $\theta_{12} \simeq 34^{\circ}, \theta_{23} \simeq 39^{\circ}$ and $\theta_{13} \simeq 9^{\circ}$; the precise best fit values differ for normal and inverted hierarchy (but the difference is smaller than the $1 \sigma$ ranges) and are given in [2, 42 44], see also [45] and [46, 47] for recent reviews. What remains unknown are

- the hierarchy of neutrino masses - One can distinguish between two non-equivalent setups. The normal hierarchy corresponds to $m_{1}<m_{2}<m_{3}$, with $\Delta m_{\mathrm{sol}}^{2}=m_{2}^{2}-m_{1}^{2}$ and $\Delta m_{\mathrm{atm}}^{2}=$ $m_{3}^{2}-m_{1}^{2} \simeq m_{3}^{2}-m_{2}^{2} \gg \Delta m_{\text {sol }}^{2}$. The inverted hierarchy corresponds to $m_{2}^{2}>m_{1}^{2}>m_{3}^{2}$, with $\Delta m_{\mathrm{sol}}^{2}=m_{2}^{2}-m_{1}^{2}$ and $\Delta m_{\mathrm{atm}}^{2}=m_{1}^{2}-m_{3}^{2} \simeq m_{2}^{2}-m_{3}^{2} \gg \Delta m_{\mathrm{sol}}^{2}$.

- the CP-violating phases - The Dirac phase $\delta$ is the analogue to the CKM phase and remains present even for $M_{M}=0$. Global fits to neutrino data tend to prefer $\delta \sim \pi$ [42], but are not conclusive. The Majorana phases $\alpha_{1}$ and $\alpha_{2}$ become unphysical in the limit $M_{M} \rightarrow 0$ because they can be absorbed into redefinitions of the fields. 
- the absolute mass scale - The mass of the lightest neutrino is unknown, but the sum of masses is bound from above as $\sum_{i} m_{i}<0.23 \mathrm{eV}$ [48] by cosmology 19 . It is also bound from below by the measured mass squares, $\sum_{i} m_{i}>0.06 \mathrm{eV}$ for normal and $\sum_{i} m_{i}>0.1 \mathrm{eV}$ for inverted hierarchy.

\subsection{Neutrino masses from right handed neutrinos}

In this section we discuss how sterile neutrinos described by the Lagrangian (1) can give Dirac or Majorana mass terms to the active neutrinos. It is obvious that the Lagrangian (2) with $M_{M}=0$ represents Dirac neutrinos with a mass term (7). For $M_{M} \neq 0$ they generate a Majorana mass term (4). If the mass $M_{M}$ is sufficiently large, $\mathrm{RH}$ neutrinos are heavy and neutrino oscillation experiments can be described by an effective Lagrangian that is obtained by integrating them out of (2),

$$
\mathcal{L}=\mathcal{L}_{S M}-\frac{1}{2} \overline{\nu_{L}} m_{\nu} \nu_{L}^{c} \text { with } m_{\nu}=-m_{D} M_{M}^{-1} m_{D}^{T}
$$

If the Majorana mass is above the electroweak scale $\left(M_{M} \gg v\right)$ one can already integrate out the $\nu_{R}$ in (11) to obtain (5) with $f=F M_{M}^{-1} F^{T}$,

$$
\mathcal{L}=\mathcal{L}_{S M}+\frac{1}{2} \overline{l_{L}} \tilde{\Phi} F M_{M}^{-1} F^{T} \tilde{\Phi}^{T} l_{L}^{c}
$$

Hence, the SS with three massive neutrinos is effectively realized if $M_{M}$ is either zero or so much bigger than the observed neutrino masses that (10) can be used to describe neutrino oscillations. If one or more eigenvalues of $M_{M}$ are not that large, then the light sterile neutrinos appear as new particles in neutrino experiments. To explore the full range of masses, we write the mass term as

$$
\frac{1}{2}\left(\overline{\nu_{L}} \overline{\nu_{R}^{c}}\right) \mathfrak{M}\left(\begin{array}{c}
\nu_{L}^{c} \\
\nu_{R}
\end{array}\right)+\text { h.c. } \equiv \frac{1}{2}\left(\overline{\nu_{L}} \overline{\nu_{R}^{c}}\right)\left(\begin{array}{cc}
0 & m_{D} \\
m_{D}^{T} & M_{M}
\end{array}\right)\left(\begin{array}{c}
\nu_{L}^{c} \\
\nu_{R}
\end{array}\right)+\text { h.c. }
$$

Here we have used the identity $\overline{\nu_{R}^{c}} M_{M} \nu_{R}^{c}=\overline{\nu_{R}} M_{M}^{T} \nu_{R}$ and the symmetry of $M_{M}$. If $\nu_{R}$ exist, they generate a mass term for $\nu_{L}$ except if $F$ either vanishes20, $F$ and $M_{M}$ have a particular flavour structure that leads to cancellations in $m_{\nu} 21$ or there are not enough RH neutrino flavours $\nu_{R}$ to give mass to all $v_{I} 22$; this is seen as a strong motivation to postulate their existence 23 . The relative size of $M_{M}$ and $m_{D}$ (i.e. their eigenvalues) allows to distinguish different scenarios. Note that this does not say anything about the absolute scale of $m_{D}$, which may lie anywhere between 0 and the electroweak scale (assuming perturbative couplings $F$ ).

\footnotetext{
${ }^{19}$ The bound and preferred value for $\sum_{i} m_{i}$ slightly change, depending on the dataset and analysis. It becomes more stringent when different data sets are combined, see e.g. [49].

${ }^{20}$ In the case $F=0$ it is not clear from the viewpoint of (1) why the fields $\nu_{R}$ should be called "neutrinos", as they have nothing in common with the known neutrinos except being neutral. Of course, such pariah neutrinos [which only interact gravitationally in (1)] can be charged in a way that justifies this classification under an extended gauge group that it broken at high energies.

${ }^{21}$ Such cancellation occurs in models where $M_{M}$ and $F$ are chosen in a way that there is no total lepton number violation.

${ }^{22}$ For the Lagrangian (1) the number of massive active neutrinos $v_{L, \alpha}$ cannot exceed the number $n$ of RH fields $\nu_{R, I}$; each $\nu_{R, I}$ can only generate one neutrino mass. Hence, at least two RH neutrino flavours are required to explain the observed mass splittings $\Delta m_{\mathrm{atm}}$ and $\Delta m_{\mathrm{sol}}$ (for $n=2$ the lightest active neutrino is massless).

${ }^{23}$ Of course there are alternative ways to generate a neutrino mass term, see e.g. [50 65] or [24 26] for a general overview with many references.
} 
$\boldsymbol{M}_{\boldsymbol{M}} \ll \boldsymbol{m}_{\boldsymbol{D}}$ - This is a pseudo-Dirac scenario; for $n=3$ the neutrinos in this case effectively behave like Dirac particle 24 . This case is excluded unless $M_{M} \lesssim 10^{-9} \mathrm{eV}$, as otherwise solar neutrino oscillations into $\nu_{R}$ should have been observed [66].

$M_{M} \sim m_{D}$ - In this case $M_{M}$ and $m_{D}$ should both be of the order of the observed neutrino mass differences. The full mass term can be diagonalized by a $(3+n) \times(3+n)$ matrix $\mathcal{U}$ as $\mathfrak{M}=\mathcal{U} \operatorname{diag}\left(m_{1}, m_{2}, m_{3}, M_{1}, \ldots, M_{n}\right) \mathcal{U}^{T}$. This can lead to rather large mixing between $\nu_{L}$ and $\nu_{R}$.

$\boldsymbol{M}_{\boldsymbol{M}} \gg \boldsymbol{m}_{\boldsymbol{D}}$ - This is the seesaw limit, in which the neutrino mass term is described by (10). This scenario is discussed in detail in the following section 2.3. The seesaw limit roughly applies if $M_{M}>1 \mathrm{eV}$.

\subsection{The seesaw mechanism}

In the limit $m_{D} \ll M_{M}$ (in terms of eigenvalues), the full $(3+n) \times(3+n)$ mass matrix $\mathfrak{M}$ for $\nu_{L}$ and $\nu_{R}$ has two distinct sets of eigenvalues. $n$ of them $\left(M_{I}\right)$ are of the order of the eigenvalues of $M_{M}$, the remaining three $\left(m_{i}\right)$ are suppressed by two powers of the active - sterile mixing matrix $\theta$

$$
\theta=m_{D} M_{M}^{-1} .
$$

The seesaw hierarchy $\theta \ll 1$ separates the two distinct sets of mass eigenstates. One can blockdiagonalize (12) by expanding in $\theta$; on one hand one obtains the $3 \times 3$ mass matrix

$$
m_{\nu}=-\theta M_{M} \theta^{T}
$$

with eigenvalues $m_{i}$; it corresponds to the operator (10). The known bounds on neutrino masses impose constraints between the values of $F$ and $M_{M}$, see figure 2. On the other hand there is the $n \times n$ matrix

$$
M_{N}=M_{M}+\frac{1}{2}\left(\theta^{\dagger} \theta M_{M}+M_{M}^{T} \theta^{T} \theta^{*}\right)
$$

with eigenvalues $M_{I}$. The matrices $m_{\nu}$ and $M_{N}$ are not diagonal and lead to flavour oscillations. Diagonalizing them yields the mass term

$$
\frac{1}{2}\left(\overline{v_{L}} m_{\nu}^{\operatorname{diag}} v_{L}^{c}+\overline{v_{R}^{c}} M_{N}^{\operatorname{diag}} v_{R}\right)+h . c .
$$

with

$$
m_{\nu}^{\operatorname{diag}}=\operatorname{diag}\left(m_{1}, m_{2}, m_{3}\right), M_{N}^{\text {diag }}=\left(M_{1}, \ldots, M_{n}\right)
$$

In summary, the full matrix $\mathcal{U}$ that diagonalizes $\mathfrak{M}$ reads

$$
\mathcal{U}=\left[\left(\begin{array}{cc}
\mathbb{1}-\frac{1}{2} \theta \theta^{\dagger} & \theta \\
-\theta^{\dagger} & \mathbb{1}-\frac{1}{2} \theta^{\dagger} \theta
\end{array}\right)+\mathcal{O}\left[\theta^{3}\right]\right]\left(\begin{array}{cc}
U_{\nu} & \\
& U_{N}^{*}
\end{array}\right) .
$$

Three mass states have light masses $m_{i} \sim \mathcal{O}\left[\theta^{2} M_{M}\right]$ and are mainly mixings of the $\mathrm{SU}(2)$ charged fields $\nu_{L}$,

$$
v_{L} \equiv U_{\nu}^{\dagger}\left(\left(\mathbb{1}-\frac{1}{2} \theta \theta^{\dagger}\right) \nu_{L}-\theta \nu_{R}^{c}\right)
$$

\footnotetext{
${ }^{24}$ This is not only way how Dirac fermions can arise. For instance, if the splitting between two eigenvalues of $M_{M}$ is sufficiently small, then one can form Dirac spinor by combining different flavours, see appendix B.
} 
The remaining $n$ mass states have masses $M_{I} \sim \mathcal{O}\left[M_{M}\right]$ and are mainly mixings of the singlet fields $\nu_{R}$,

$$
v_{R} \equiv U_{N}^{\dagger}\left(\left(\mathbb{1}-\frac{1}{2} \theta^{T} \theta^{*}\right) \nu_{R}+\theta^{T} \nu_{L}^{c}\right) .
$$

We refer to $v_{L}$ as active neutrinos because they take part in the unsuppressed weak interactions (3). The $v_{R}$ also participate in the weak interaction (3), but only with an amplitude suppressed by $\theta$; hence they are sterile neutrinos. The matrix $U_{N}$ diagonalises the sterile neutrino mass matrix $M_{N} 25$ it can be seen as analogue to $U_{\nu}$. The diagonal elements of $M_{N}$ are much bigger than the off-diagonals and very close to the entries of $M_{M}$. Therefore one can in most cases neglect all terms of second order in $\theta$ and approximate $M_{N}=M_{M}, U_{N}=\mathbb{1}$. However, if two eigenvalues of $M_{M}$ are degenerate, then the $U_{N}$ can contain large sterile-sterile mixing angles. The active-sterile mixing angles are determined by the entries of the matrix $\theta$ and always small. More precisely, the experimentally relevant mixing between active and sterile species is given by the matrix $\Theta$ with 26

$$
\Theta_{\alpha I} \equiv\left(\theta U_{N}^{*}\right)_{\alpha I}
$$

Practically, experiments to date constrain the quantities

$$
U_{\alpha}^{2} \equiv \sum_{I} \Theta_{\alpha I} \Theta_{\alpha I}^{*}=\sum_{I} \theta_{\alpha I} \theta_{\alpha I}^{*}
$$

and combinations thereof.

This is known as the seesaw mechanism [67 70] because increasing the eigenvalues of $M_{M}$ pushes the masses of the sterile neutrinos up and those of active neutrinos down, just as if they sat on a seesaw 27. If the couplings $F$ are of order one and $M_{M}$ in the range suggested by GUT models, then $m_{\nu}$ roughly reproduces the observed neutrino mass splittings. Hence, the mechanism provides a natural explanation for the smallness of neutrino masses. However, the scale $M_{M}$ of the seesaw is phenomenologically almost unconstrained and may be as low as $1 \mathrm{eV}$ [75].

To make the Majorana nature of the fields explicit (and get rid of the charge conjugation matrix in the mass term) one can describe them in terms of Majorana spinors; we define the flavour vectors 28

$$
N \equiv v_{R}+v_{R}^{c}, v \equiv v_{L}+v_{L}^{c} .
$$

Obviously, the elements $v_{i}$ are active neutrinos and the $N_{I}$ sterile neutrinos. The mass and kinetic terms then can be combined as

$$
\frac{1}{2} \bar{N}\left(i \not \partial-M_{N}^{\text {diag }}\right) N+\frac{1}{2} \bar{v}\left(i \not \partial-m_{\nu}^{\text {diag }}\right) v
$$

Up to the normalization, this looks like the Lagrangian for a Dirac field, but one has to keep in mind the Majorana conditions $N_{I}=N_{I}^{c}, v_{i}=v_{i}^{c}$.

\footnotetext{
${ }^{25}$ We choose the phase convention in $U_{N}$ such that $M_{N}=U_{N}^{*} \operatorname{diag}\left(M_{1}, \ldots, M_{n}\right) U_{N}^{\dagger}$ with $M_{I}$ real and positive. In this convention we observe the same relations $\nu_{L} \simeq U_{\nu} v_{L}$ and $\nu_{R} \simeq U_{N} v_{R}$ for left and right handed fields (rather than $U_{N}^{*}$ for $\left.\nu_{R}\right)$.

${ }^{26}$ In (20) the matrix $U_{N}^{\dagger} \theta^{T}=\Theta^{T}$ appears (rather than $\Theta$ ) because the $N_{I}$ couple to $\nu_{L, \alpha}$, but overlap with $\nu_{L, \alpha}^{c}$.

${ }^{27}$ More precisely, this is the type-I seesaw mechanism. The term "seesaw" is also used for several modifications of this idea 71], such as the "type-II seesaw" [52, 54 57], "type-III" seesaw [60], "split seesaw" 72], "radiative seesaw" 73, 74] or "inverse seesaw" [58, 59].

${ }^{28}$ Obviously $v_{R}=P_{R} N$ and $v_{L}=P_{L} v$, where $P_{R, L}$ are chiral projectors.
} 


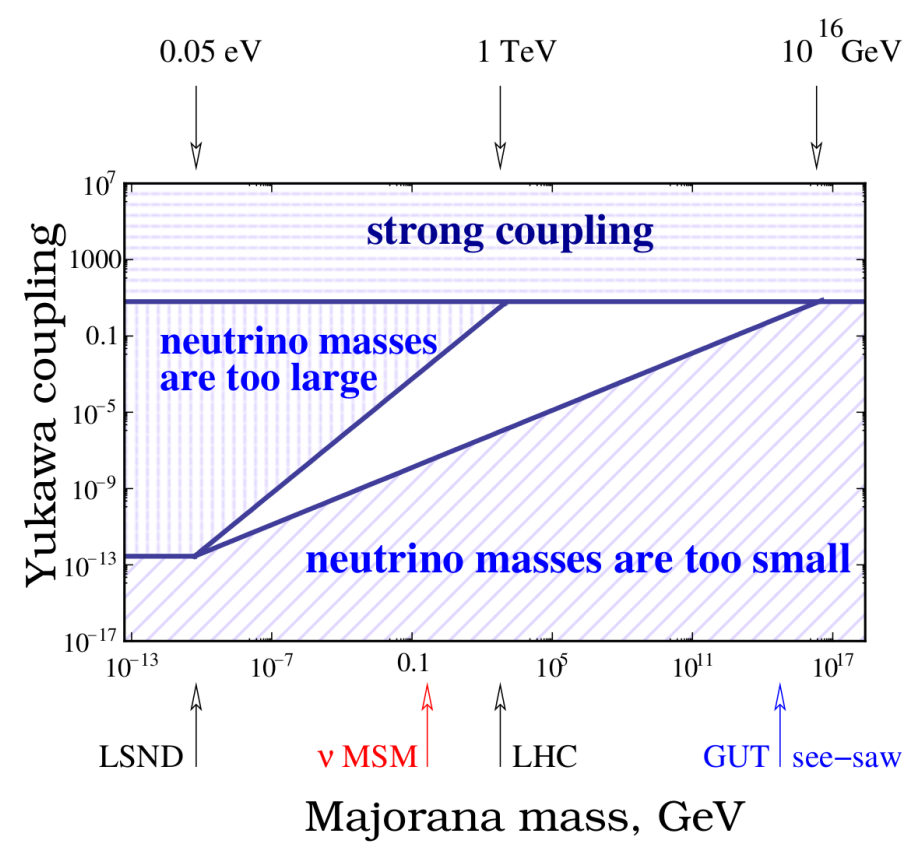

Figure 2: A schematic illustration of the relation between $F$ and $M_{M}$ in the seesaw limit $m_{D} \ll M_{M}$. Individual elements of the matrices $F$ and $M_{M}$ can deviate considerably from this if there are cancellations in (14). Plot taken from [27].

\section{Other laboratory experiments}

The production and study of $N_{I}$ particles in the laboratory is in principle possible if $M_{I}$ is below the electroweak scale. At energies $\ll M_{I}$, the $N_{I}$ only leave indirect traces in the laboratory. They manifest as higher dimensional operators [76], such as the mass term (10). These can lead to deviations from SM predictions in different observables, such as lepton number violation or $\beta$-decays. These signatures provide valuable information, but are usually not specific to $\mathrm{RH}$ neutrinos. Here we list a number of experimental setups that can constrain the properties of $N_{I}$. So far almost all but those in section 3.1 have reported negative results, i.e. only allow to exclude certain parameter regions.

\subsection{Neutrino oscillation anomalies}

Accelerator experiments - Some short baseline and reactor neutrino experiments have reported deviations (ii) from the SS. A more detailed review of these results can found in [77], which we follow closely here. The most prominent findings come from the LNSD experiment [78, 79], which studied transitions $\bar{\nu}_{\mu} \rightarrow \bar{\nu}_{e}$ and saw an excess of $\bar{\nu}_{e}$-events. The similar KARMEN experiment did not see such excess [80], but could not exclude the entire parameter space of the LSND anomaly [81], mostly due to its shorter baselength. The most recent results from the MiniBooNE experiment [82] from $\nu_{\mu} \rightarrow \nu_{e}$ and $\bar{\nu}_{\mu} \rightarrow \bar{\nu}_{e}$ studies seem compatible with the LSND anomaly, which is in contrast to previous results. However, it has been pointed out that much of the signal comes from energies below $475 \mathrm{MeV}$, where the background evaluation is problematic [77]. The ICARUS experiment [83] is also sensitive to sterile neutrinos; active-sterile oscillations get averaged over, but lead to an energy independent enhancement of event rates. 

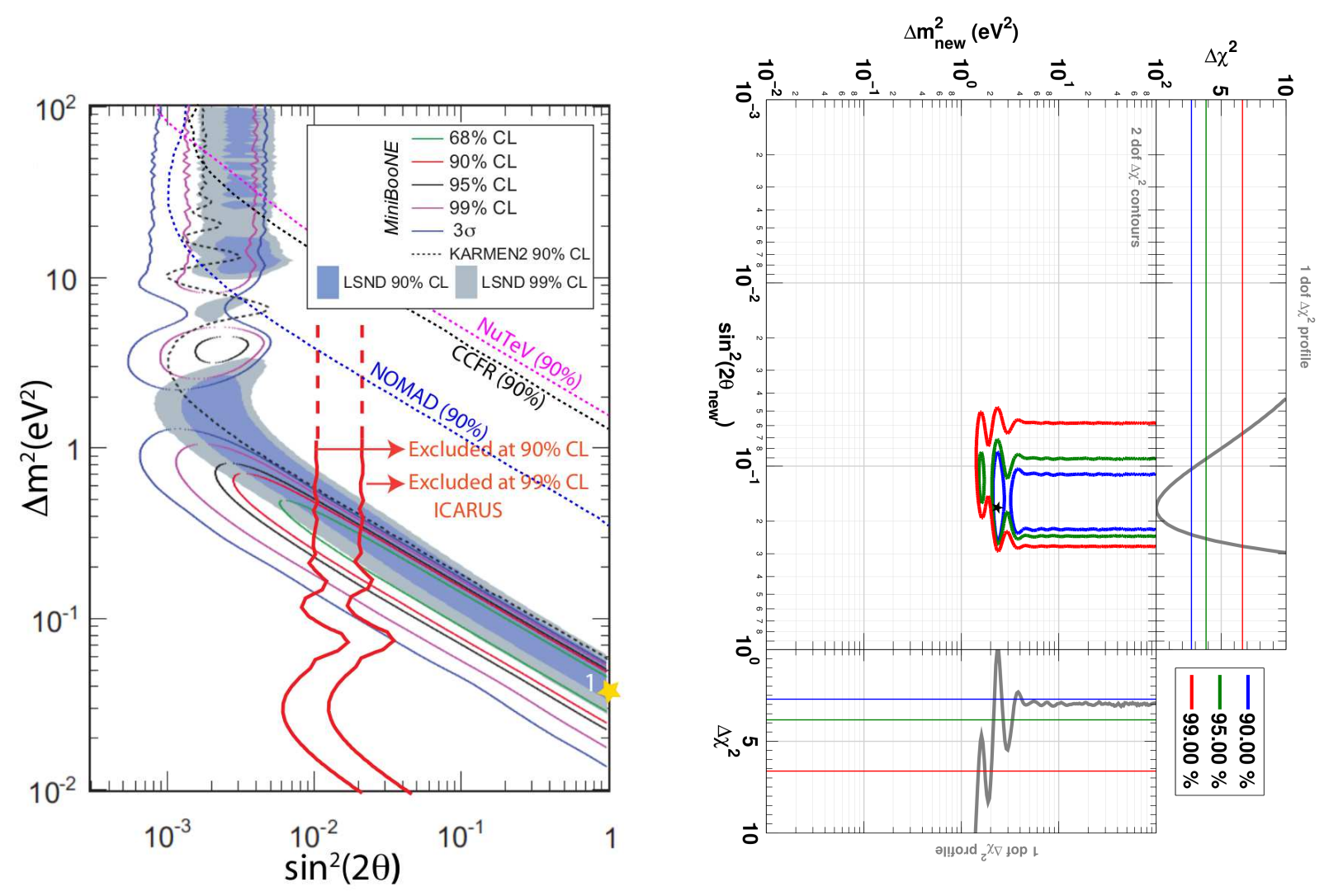

Figure 3: Left plot: The region in the mass-mixing plane preferred by the LSND anomaly (coloured bands) is compared to the region allowed by MiniBooNe (coloured lines) and exclusion plots by other experiments as indicated in the plot; plot taken from [83]. Right plot: region allowed by the combined reactor and gallium anomalies, taken from [27]. Data from both is e.g. combined in figure 4 in [84].

Together these experiments roughly restrict the parameter space for one sterile neutrino to a mass splitting $\Delta m^{2} \sim 0.5 \mathrm{eV}^{2}$ and mixing $\sin ^{2} 2 \theta \sim 5 \times 10^{-3}$ [77], see figure 3,

Reactor and gallium anomalies - There are two more anomalies that can be interpreted as a sign for sterile neutrinos with $\mathrm{eV}$ masses. One is the reactor anomaly [85]. The neutrino fluxes from nuclear reactors appeared to be in agreement with theory until recently. It was not new experimental data, but more refined theoretical calculations that lead to tension with the SS [86, 87]. However, it should be pointed out that even in these results, about $10 \%$ of the $\beta$-decay branches are not known and can only be estimated 29 This involves using the ILL-experiment's measurement for the electron spectrum [89, 90] as a reference point, hence the anomaly could be due to a systematic in that measurement. The other anomaly arises in the calibration of the GALLEX and SAGE experiments [91, 92].

These anomalies have resulted in a great interest in searches for $\mathrm{eV}$-mass sterile neutrinos.

\footnotetext{
[88.

${ }^{29}$ If the uncertainty in $\beta$-transitions is bigger than usually assumed the anomaly could be statistically insignificant
} 
Global fits to all data have e.g. been performed in [84, 93, 94]. As discussed in detail in [84], the situation at this stage is not clear. The accelerator anomalies come from appearance measurements (muon to electron). If they are caused by sterile neutrino oscillations, there should also be a $\nu_{\mu}$ disappearance, which is not observed. This tension is present in the $3+1$ (three active and one $\mathrm{eV}$ mass sterile neutrino) model and remains in $3+2$ scenarios (three active plus two $\mathrm{eV}$ mass sterile neutrinos). It reduces a bit in a $1+3+1$ model with two sterile neutrinos (where one sterile neutrino is lighter than an active one). The reactor and gallium anomalies, which are due to disappearance, do not show such tension to other experiments. They come, however, from $\nu_{e}$ disappearance, which is controlled by other parameters than $\nu_{\mu}$ disappearance. Hence, the situation remains puzzling. A detailed list of various proposals for future experiments [95 98] can be found in [27].

\subsection{Lepton flavour violation}

The most studied consequence of the lepton number violation due to $M_{M}$ is the neutrinoless double $\beta$-decay discussed in the following subsection 3.3, $M_{M}$ and $F$ also mediate flavour violation in the charged lepton sector [55, 99 109], leading e.g. to muon decays $\mu \rightarrow e \gamma$ and unitarity violation of the PMNS matrix [110, 111]. Searches for these processes in proposed experiments, such as COMET [112, 113] or Mu2e [114], can help to constrain RH neutrino properties [109].

In the framework of (1), with no other physics added to the SM, it is hard to observe these processes. For a generic choice of parameters the seesaw relation (14) implies that either the suppressing scale $M_{I}$ is too heavy or the Yukawa couplings $F$ are too small. A class of models that offers relatively good chances to observe lepton flavour violation are those where the structure of $F$ and $M_{M}$ is such that an approximately conserved generalized lepton number can be defined [58, 76, 115 121]. In this case the scales where lepton flavour violation and total lepton number violation occur can be rather different. In such models even $\mathrm{RH}$ neutrinos responsible for the baryon asymmetry of the universe may give a measurable contribution to $\mu \rightarrow e \gamma[121] 30$

Models that embed (1) into an extended framework, such as supersymmetric and grand unified theories, often contain interactions that violate lepton flavour or even the total lepton number. This includes left-right symmetric models, see e.g. [124] and references therein. It also applies to various "bottom up" models that e.g. include an extended scalar sector which couples to neutrinos. Hence, while non-observation of $\mu \rightarrow e \gamma$ and other processes can constrain $N_{I}$-properties, the observation would not necessarily be a clear sign for their existence.

\subsection{Neutrinoless double $\beta$-decay}

If neutrinos are Majorana particles, neutrinoless double- $\beta$ decays $(0 \nu \beta \beta)$ are possible [57] (see [125, 126] for recent reviews). Whether $0 \nu \beta \beta$-decay occurs at observable rates depends on the Majorana mass matrix $M_{M}$. The lepton number violation is sufficient if at least one eigenvalue of $M_{M}$ is larger than the exchanged momentum $\sim 100 \mathrm{MeV}$ [127 129], see also [106, 130]. The $0 \nu \beta \beta$-decay can be pictured as exchange of an electron neutrino that acts as its own antiparticle between the nuclei, see figure 4. The amplitude is given by the convolution of nuclear matrix

\footnotetext{
${ }^{30}$ Right handed neutrinos can also mediate lepton number violation in more exotic models. For instance, $\mu \rightarrow e \gamma$ may be measurable 122] in models where the $\nu_{R}$ are composite objects that are hold together by strong couplings in a hidden sector [37, 123].
} 


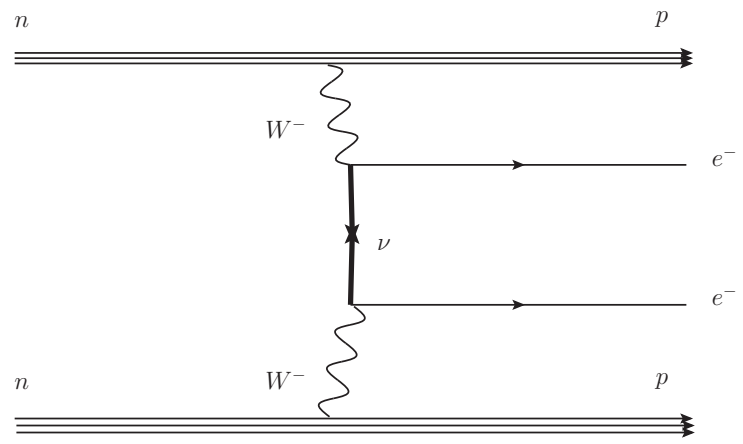

Figure 4: Diagram for neutrinoless double beta decay. Here we have made the lepton number flow explicit by assigning arrows to fermion lines. The "clashing arrows" in the center of the diagram are allowed because neutrinos and antineutrinos are indistinguishable if they are Majorana particles. If some $N_{I}$ are light enough, they may also be exchanged instead of $v_{i}$. The amplitude for this process vanishes in the limit $M_{M} \rightarrow 0$.

elements with

$$
\left(U_{\nu}\right)_{e i}^{2} \frac{m_{i}^{2}}{p^{2}-m_{i}^{2}}+\Theta_{e I}^{2} \frac{m_{I}^{2}}{p^{2}-m_{I}^{2}}
$$

Here $p^{2}=p_{0}^{2}-\mathbf{p}^{2}$ is the exchanged momentum, with $|\mathbf{p}| \sim 100 \mathrm{MeV}$. In the regimes $M_{I} \ll 100$ $\mathrm{MeV}$ and $M_{I} \gg 100 \mathrm{MeV}$ one can make analytical approximations and approximate the inverse half life time for $0 \nu \beta \beta$-decays

$$
\tau^{-1} \simeq G_{0 \nu}\left|\sum_{i} \mathcal{M}_{i}\left(U_{\nu}\right)_{e i}^{2} \frac{m_{i}}{m_{e}}+\sum_{I}^{M_{I} \ll 100 \mathrm{MeV}} \mathcal{M}_{I} \Theta_{e I}^{2} \frac{m_{i}}{m_{e}}+\sum_{I}^{M_{I} \gg 100 \mathrm{MeV}} \tilde{\mathcal{M}}_{I} \Theta_{e I}^{2} \frac{m_{p}}{m_{i}}\right| .
$$

Here $G_{0 \nu}$ is the "phase space factor" (for instance $G_{0 \nu}=7.93 \times 10^{15} \mathrm{yr}^{-1}$ for ${ }^{76} \mathrm{Ge}[129]$ ). The dependence in the intermediate regime is more complicated [129]. The $\mathcal{M}_{i}$ and $\mathcal{M}_{I}$ are nuclear matrix elements corresponding to light active or sterile neutrino exchange. The $\tilde{\mathcal{M}}_{I}$ are matrix elements for the contribution from heavy sterile neutrinos, sometimes called "direct contribution" or "contact term" (the $\sim$ indicates the different normalization conventions for both regimes). Such heavy neutrinos are not really "exchanged" as propagating particles; because their mass is larger than the exchanged momentum, they can be integrated out and their contribution can be understood in terms of an effective operator as (44). The nuclear matrix elements are a source of considerable uncertainty.

Usually light neutrinos (active or sterile) with masses $\ll 100 \mathrm{MeV}$ strongly dominate in the exchange [131]. However, without any extra sources of $L$-violation beyond (11), the existence of a mass state with $M_{I}>100 \mathrm{MeV}$ remains a necessary condition for observable rates of $0 \nu \beta \beta$-decay even if the direct exchange of these heavy particles does not give a significant contribution to $\tau^{-1}$. When only light neutrinos are exchanged one can approximately factorize the dependencies on nuclear and neutrino physics and parametrize the latter in terms of the effective Majorana mass 31

$$
m_{e e}=\left|\sum_{i}\left(U_{\nu}\right)_{e i}^{2} m_{i}+\sum_{I}^{M_{I} \ll 100 \mathrm{MeV}} \Theta_{e I}^{2} M_{I}\right|
$$

\footnotetext{
${ }^{31}$ The effective Majorana mass is different from the kinetic mass $\left(\sum_{i}\left|\mathcal{U}_{e i}\right|^{2} m_{i}^{2}\right)^{1 / 2}$.
} 


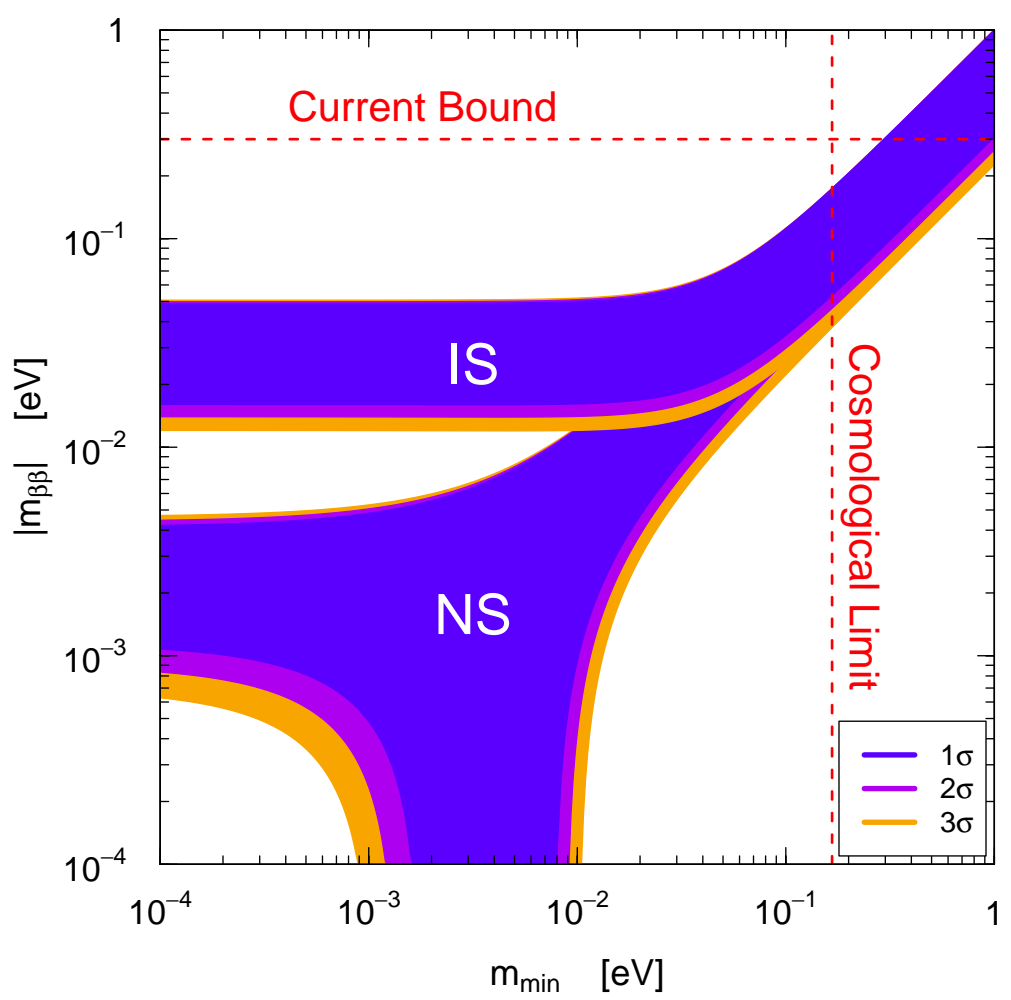

Figure 5: Current bounds on $m_{e e}$ (here called $m_{\beta \beta}$ ) as a function of the smallest neutrino mass, as summarized in [126]. "NS" refers to normal hierarchy, "IS" to inverted hierarchy. The cosmological limit has tightened with the publication of the Planck results [48].

For very light $N_{I}$ one has to go beyond the seesaw approximation (21) to calculate the activesterile mixing matrix $\Theta$.

Leaving aside some tuned special cases 131], the contribution from active neutrinos dominates in (type I) seesaw models [129, 132 134]. If all sterile neutrino masses are below $100 \mathrm{MeV}$, they usually give a negative contribution, i.e. reduce the rate of $0 \nu \beta \beta$-decays compared to the case where only active neutrinos are exchanged (which in any case is undetectably small if there is no $M_{I}$ above $100 \mathrm{MeV}$ ), see [135] for a recent discussion. The existence of light sterile neutrinos is strongly constrained by cosmology. In particular, if sterile neutrinos compose the observed DM (see section 6), then the bounds on their mixing imply that they do not contribute [132, 136]. If only the active neutrino mass states $v_{i}$ contribute to (26) and have an inverted mass hierarchy, then there exists a lower bound $m_{e e}>20 \mathrm{meV}$, see figure 5. For normal hierarchy there is no such bound. If also light sterile states $N_{I}$ contribute [57, 129, 132, 133, 136 138] there is no lower bound for any hierarchy.

So far there is no clear observation of neutrinoless double- $\beta$ decays. The only claim of a detection [8, 139] suggests $m_{e e}=0.32 \pm 0.03 \mathrm{eV}$ [140], but is disputed, as it appears to be in conflict with other observations [141-143]. For instance, the EXO-200 experiment finds $m_{e e}<0.14-0.38$ $\mathrm{eV}$ [144]. Other experiments so far only put upper bounds on the rate for this process, see figure 5 (an overview is also given in [144, 145]). 


\subsection{Collider searches}

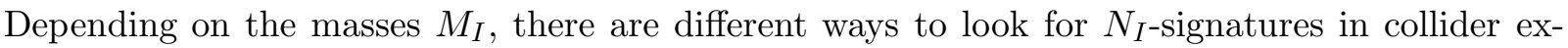
periments, see [146] for a review.

Intensity frontier - If kinematically possible, the sterile neutrinos $N_{I}$ participate in all pro-
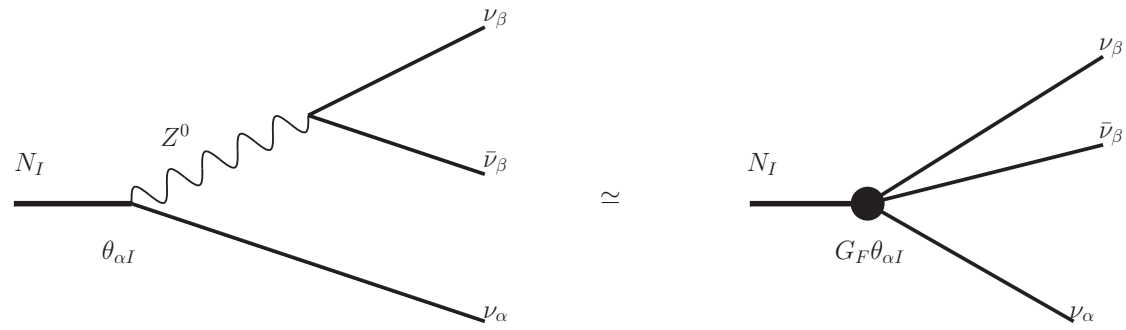

Figure 6: Example for a contribution to $N_{I}$-decay at low energies. The $N_{I}$ itself can be produced in meson decays. Instead of the $\bar{\nu}_{\beta}-\nu_{\beta}$ pair there can also be charged lepton-antilepton pairs or quark-antiquark pairs (which hardronize) in the final state if this in kinematically allowed.

cesses that involve active neutrinos, but with a probability that is suppressed by the small mixings $U_{\alpha}^{2}$. This makes it possible to produce them in meson decays for $M_{I} \lesssim$ a few GeV $\left.147-152\right]$, see also $[153-155]]^{32}$. One can distinguish two ways to look for $N_{I}$. First, they could be seen as missing energy in the meson decays that produce them; in two body decays this allows to determine their mass and (via the branching ratio) their mixing. Second, the subsequent decay of the $N_{I}$ shown in figure 6 may also be observed (e.g. as "nothing $\rightarrow$ leptons" process) if one places a detector along the beamline. For some parameter choices, it can even be possible to observe both events in the same detector [157]. Several experiments of this type have been set up in the past 158 165], in particular CERN PS191 [160, 161], NuTeV [162], CHARM [163], NOMAD [164] and WA66 [165]. The bounds derived from these for $n=2$ are shown in figure 11. They are valid under the assumption that $N_{I}$ have no interactions other than those in (11) below the electroweak scale. If the mass $M_{I}$ is larger than a few $\mathrm{GeV}$, the $N_{I}$ are too heavy to be produced efficiently ( $\mathrm{D}$ or $\mathrm{B}$ meson decays are not possible) and it is unlikely that direct searches in the near future can find them. If they are, on the other hand, lighter than $\sim \mathrm{MeV}$, then their Yukawa coupling $F$ must be very small due to (14) and the branching ration is very small 33 .

High energy frontier - Sterile neutrinos with masses up to a few $\mathrm{TeV}$ in principle are within reach of the LHC or other future high energy experiments [18, 119, 146, 166 181]. A promising signal in different scenarios are $N_{I}$-decays that involve same sign dileptons (two leptons of the same charge) in the final state. However, the Yukawa coupling in (11) have to be tiny due to the seesaw relation (14) and $\mu \rightarrow e \gamma$ data [106. If the $\nu_{R}$ only interact via these Yukawa couplings $F$, the branching ratio is usually too small [175]; a detection is only possible if $F$ has a particular structure that leads to cancellations in the contributions of different elements to $m_{\nu}$ [118, 119], hence allows for larger individual entries of $F$. Such cancellation can occur if the total lepton number $L$ is approximately conserved [118]. If the $\nu_{R}$ only interact via these Yukawa couplings $F$,

\footnotetext{
${ }^{32}$ Sterile neutrinos may also be produced in the decay of tauons [154 156$]$.

${ }^{33}$ It has been suggested that, if $M_{M}$ is generated by spontaneous symmetry breaking below the electroweak scale, it may be possible to detect even lighter (keV) sterile neutrinos [166].
} 
the branching ratio is usually too small [175]; a detection is only possible if $F$ has a particular structure that leads to cancellations in the contributions of different elements to $m_{\nu}$ [118, 119], hence allows for larger individual entries of $F$. Such cancellation can occur if the total lepton number $L$ (summed over active and sterile flavours) is approximately conserved [118].

The perspectives are much better if the $\nu_{R}$ have interactions in addition to those in (11). For instance, it may be possible to study $\mathrm{RH}$ neutrinos in high energy collisions if the scalar sector is extended in comparison to the SM [166, 171, 174, 177], in models where neutrino masses are generated via the inverse seesaw mechanism [178, 179, 182] or if spacetime has more than four dimensions [176, 183]. In left - right symmetric models [184 186] the $\nu_{R}$ are charged under a right chiral $S U(2)$ gauge symmetry, which can be broken near the $\mathrm{TeV}$ sale. One promising possibility to test such models is to search for the associated gauge bosons [187], but also the properties of the $N_{I}$ themselves can be probed [188 191]. Current bounds are given in [192, 193], the right $W$-boson should be heavier than about $2.5 \mathrm{TeV}[124]$. In supersymmetric (SUSY) theories the seesaw mechanism may also be studied indirectly at colliders [194]; if their SUSY-partner (sneutrino) is the lightest sparticle, then observations of the decays of heavier SUSY-particles into sterile sneutrinos can constrain their properties [195]. It has been suggested that $N_{I}$ can give a contribution to the Higgs mass, see e.g. [196 198], though this is disputed [199]. Finally, LHC searches can also be promising for models that couple $N_{I}$ to $Z^{\prime}$ gauge bosons [200, 201], including those where $N_{I}$ are responsible for leptogenesis [202].

\subsection{Direct dark matter searches}

Sterile neutrinos are a promising DM candidate, which we discuss in detail in section 6, If they compose all DM, then bounds from X-ray and structure formation imply that their Yukawa couplings are so small that they cannot contribute to the observed neutrino oscillations [203] $]^{34}$. This means that, if one at the same time requires $\mathrm{RH}$ neutrinos to explain the two observed neutrino mass differences, there must be at least three of them. The small coupling also makes it practically impossible to directly observe these particles in collider experiments due to the tiny branching ratio for their production. They could at least in principle be found in direct detection experiments that look for interactions of DM sterile neutrinos from the interstellar medium with atomic nuclei in the laboratory, and some experiments have been suggested [205, 206]. However, such detection would be extremely challenging (most likely impossible) due to the small mixing angle and the background from solar and stellar active neutrinos [207].

\subsection{Other constraints}

Several other ways to constrain $N_{I}$ properties have been suggested. If they have eV masses as suggested by the oscillation anomalies (ii), they should affect $\beta$-decays 75 , 208 211], which has not been observed. They may also leave traces in neutrino telescopes 212] like IceCube [213 217] or detectors for direct DM searches [218]. The effect of keV sterile neutrinos on nuclear decays has been studied in [219, 220], a detection at the current stage seems very unlikely due to the background. In [221] it was found that sterile neutrinos with $\mathrm{TeV}$ masses can improve the fits to electroweak precision data 35

\footnotetext{
${ }^{34}$ It has been shown that the bounds on DM sterile neutrinos can constrain the active neutrino mass spectrum if additional assumptions are made [204].

${ }^{35}$ Electroweak precision data also allows to constrain models of right handed neutrinos with electroweak scale masses beyond (1), see e.g. [222].
} 


\section{Thermal history of the universe}

If $\mathrm{RH}$ neutrinos $\nu_{R}$ with $F \neq 0$ exist, they are necessarily produced thermally in the early universe. At temperatures above the electroweak scale $T_{E W} \sim 140 \mathrm{GeV}$ [assuming a Higgs mass of $\sim 125 \mathrm{GeV}[22,23]$ ], Higgs particles are present in the plasma. This allows for $N_{I}$ production as long as the temperature is high enough $\left[T \gtrsim M_{I}(T)\right.$, where $M_{I}(T)$ is an effective mass in the plasma]. The relevant processes are decays and inverse decays $N_{I} \leftrightarrow \Phi l_{\alpha}$ (or $\Phi \leftrightarrow N_{I} l_{\alpha}$, depending on the effective masses of the (quasi)particles in the plasma [223 225]) and scatterings (such as $\bar{t} t \leftrightarrow N_{I} l_{\alpha}$ ). If the masses $M_{N}$ are below the electroweak scale, $N_{I}$ are produced at $T<T_{E W}$ via active-sterile mixing [The same process can be viewed as a $\nu_{L}-\nu_{R}$ oscillation in the flavour basis used in (10)]. In addition to that, there may be other production mechanisms if $\nu_{R}$ have additional interactions with a hidden sector or extended Higgs sector, couple directly to the inflaton or are charged under a gauge symmetry that is broken above the electroweak scale. The presence of $N_{I}$ in the plasma can have different effects in the early universe, which we will summarize in the following.

All astronomical observations to date are in rather good agreement with the $\Lambda$ CDM model of cosmology 36 , sometimes dubbed the "Concordance Model" or "Standard Model of Cosmology" in analogy to the SM. The most important cosmological parameters in the context of RH neutrinos are the fractions of the total energy budget of the observable universe from baryons $\left(\Omega_{B} \sim 0.049\right)$ and dark matter $\left(\Omega_{D M} \sim 0.265\right.$ without active neutrinos) [48], as well as the effective number of neutrino species $N_{\text {eff }}$ in the radiation dominated epoch 37 . The latter can be understood as a measure for the expansion history of the universe. The (Hubble) rate of the universe's expansion is given by

$$
H^{2}=\frac{8 \pi}{3} G \rho,
$$

where $G=M_{P}^{-2}$ is Newton's constant and $\rho$ the energy density of the universe. The contribution of "known neutrinos plus unknown physics" to $\rho$ is usually parametrized as $N_{\text {eff }} \times \rho_{\nu}$, where $\rho_{\nu}$ is the contribution from one ultrarelativistic species and $N_{\text {eff }}$ is the effective number of neutrino species. It can be identified with the actual number of neutrino species if all neutrinos are effectively massless and there is no other "new physics". It is common to parametrize

$$
\rho_{\gamma}+\rho_{\text {neutrinos }}+[\text { new physics effects }] \equiv \rho_{\gamma}+N_{\text {eff }} \rho_{\nu}=\frac{\pi^{2}}{15} T_{\gamma}^{4}\left[1+N_{\text {eff }} \frac{7}{8}\left(\frac{4}{11}\right)^{4 / 3}\right] \text {, }
$$

where $T_{\gamma}$ and $\rho_{\gamma}$ are temperature and energy density of photons and $\rho_{\text {neutrinos }}$ is the energy density of the SM-neutrinos. The second equality holds only for $T<0.2 \mathrm{MeV}$, i.e. after electrons and positrons annihilated. This is the temperature regime where $N_{\text {eff }}$ is practically tested by observations. In the standard scenario $N_{\text {eff }}=3$ during $\mathrm{BBN}$ and $N_{\text {eff }}=3.046$ at the time of photon decoupling 38 This assumes that each neutrino flavour has two internal degrees of freedom. In the SM (without neutrino masses), these are particles and antiparticles. In contrast to charged leptons and quarks, $\nu_{L}$-states do not have an independent spin degree of freedom in

\footnotetext{
${ }^{36} \mathrm{~A}$ brief review of the $\Lambda \mathrm{CDM}$ model is given in [2], see e.g. [226, 227] for detailed introductions.

37 "Radiation dominated epoch" refers to the period in the universe's history when more energy was stored in relativistic than in nonrelativistic degrees of freedom.

${ }^{38}$ The deviation from 3 parametrises a deviation from the equilibrium distribution of neutrinos caused by $e^{ \pm}$ annihilation 228].
} 
the SM: For massless particles, the helicity must be equal to the chirality; hence neutrinos (i.e. one-particle states $\left.\left|\nu_{L}\right\rangle\right)$ must have left helicity and antineutrinos have right helicity.

If additional particles were present in the early universe, this would lead to a larger value of $N_{\text {eff }}$ [229, 230]. Each $\nu_{R, I}$ adds two neutrino degrees of freedom. If $n=3$ and all of these were thermalized, $N_{\text {eff }}$ would be 6 . However, if neutrinos are Dirac fields, then the Yukawa couplings $F$ must be tiny to explain the smallness of the neutrino masses. Then the $\nu_{R}$ degrees of freedom do not get populated significantly by thermal production. In other words, the helicity changing processes are so suppressed at $T \gg m_{i}$ that practically all neutrinos, which are produced by the weak interaction, have left helicity. This argument of course assumes that $\nu_{R}$ have no other couplings than $F$ that could contribute to thermal production. Hence, the fact that we observe $N_{\text {eff }} \simeq 3-4$ strongly constrains models of Dirac neutrinos in which $\nu_{R}$ are charged under some gauge group at high energies or otherwise produced in the early universe. If neutrinos are Majorana fields and $M_{M} \gtrsim 100 \mathrm{MeV}$, then the heavy particles $N_{I}$ have decoupled and decayed long before big bang nucleosynthesis $(\mathrm{BBN})$ and do not affect light element abundances or the CMB. Lighter sterile neutrinos can be long lived enough to contribute to $N_{\text {eff }}$ during BBN and afterwards. If they are in thermal equilibrium and ultrarelativistic, then each $N_{I}$ increases $N_{\text {eff }}$ by one. If their abundance is below equilibrium or their mass not negligible, they contribute less.

Since (28) assumes that all neutrinos are massless and in thermal and chemical equilibrium, any deviation of the momentum distribution from $\left(e^{\mathbf{p} / T}+1\right)^{-1}$ [such as a chemical potential, a non-negligible mas 39 or a nonequilibrium distribution] leads to non-integer contribution to $N_{\text {eff }}$; this applies to both, active and sterile neutrinos. Furthermore, in the SM the ratio of the temperatures of the neutrino and photon backgrounds at the time of photon decoupling is $(4 / 11)^{1 / 3}$. If this ratio is different (e.g. because some decaying particle injects energy into either of them), then this would lead to deviations from $N_{\text {eff }}=3.046$ even if there are only three neutrinos [234 236].

\subsection{A brief history of the universe}

Observations of the $\mathrm{CMB}$ show that the universe was homogeneous and isotropic to one part in $\sim 100000$ at redshift $z \sim 1100$, when photons decoupled from the primordial plasma [237]. This is puzzling, as the radiation we receive from different directions originates from regions that were causally disconnected at that time if the universe only contained radiation and matter ("horizon problem"). Furthermore, the inferred overall spatial curvature is zero or very small [237], which means that it was extremely close to zero at earlier times ("flatness problem"). Both problems can be understood as the result of cosmic inflation [238, 239], a phase of accelerated expansion in the universe's very early history. Inflation can also explain the small density perturbations that served as seeds for structure formation in the universe as quantum fluctuations that were "streched out" to macroscopic scales by the rapid expansion, and predict the correct properties of their spectrum. However, while inflation is an excellent model for cosmology, we do not know much about the fundamental physics mechanism that drove it.

If inflation was driven by the potential energy of an inflaton field, then the quantum fluctuations of this field lead to small perturbations in the (otherwise homogeneous) gravitational potential. After the inflaton dissipates its energy into relativistic particles during cosmic re-

\footnotetext{
${ }^{39}$ See [231, 232] for a detailed discussion of the effects of neutrino masses in cosmology. For eV masses the deviation is small in the early universe. An early discussion in Russian can be found in [233].
} 
heating 240 242] 40 these lead to density fluctuations in the primordial plasma, which manifest in temperature fluctuations in the $\mathrm{CMB}$ and form the seeds for the formation of structures in the universe [244]. The power spectrum of CMB fluctuations is in very good agreement with the above hypothesis [237]. After reheating, $N_{I}$ can affect the thermal history of the expanding universe in several ways.

Baryogenesis via leptogenesis $\left(T>T_{E W}\right)$ - There is good evidence [245] that the observed $\Omega_{B}$ is the thermal relic of a small matter-antimatter asymmetry of order $\sim 10^{-10}$ in the early universe (BAU), which survived after all other particles and antiparticles annihilated into CMB photons and neutrinos and is reflected in today's baryon to photon ratid $\eta_{B} \sim 10^{-10}$. If this asymmetry was produced by leptogenesis, then the observed $\Omega_{B}$ allows to constrain $F$ and $M_{M}$, cf. section 5 ,

Dark Matter production $(T \sim 100 \mathrm{MeV}$ if produced by mixing) - If DM consists of thermally produced $N_{I}$, then there are various different bounds on their properties, which we discuss in section 6 .

Late time leptogenesis $\left(T_{E W}>T>\right.$ few $\left.\mathrm{MeV}\right)$ - Sphaleron processes [246] are the only source of baryon number violation [247 249] in the early universe in (1). They freeze out 42 at $T \sim T_{E W}$, below which baryon number is conserved. The production of a lepton asymmetry can, however, continue afterwards if some $N_{I}$ are out of equilibrium (e.g. during their freezeout and decay) because $F$ and $M_{M}$ violate flavoured and total lepton number, respectively. The generated asymmetries can be much bigger than $\eta_{B}$ and differ in each flavour [250, 251]. A weak constraint on the asymmetry may be derived from its effect on hadronisation at $T \sim 200 \mathrm{MeV}$ [252]. Stronger constraints can be derived from BBN, see below. Late time asymmetries can be very important if there are long lived sterile neutrinos because they can amplify or suppress their production rate. For keV-mass sterile neutrinos an amplification of their production rate [253, 254] can be so efficient that they are abundant enough to constitute all DM [250]. A suppression of the thermal production rate, on the other hand, can help to ease the tension between cosmological and laboratory hints for eV-mass sterile neutrinos, see section 4.2. In return, light sterile neutrinos can also amplify an active neutrino asymmetry [255].

Neutrino freezeout $(T \sim 1.1 \mathrm{MeV}$ for active neutrinos $)$ - The freezeout of active neutrinos leads to a cosmic background of relativistic neutrinos, analogue to the CMB. They affect the expansion by their energy density $\rho_{\text {neutrinos }}$. The neutrino background may carry a lepton asymmetry that is orders of magnitude larger than the baryon asymmetry $\sim \eta_{B} \sim 10^{-10}$. The main constrains on such asymmetry come from BBN [256]. In the SS, active neutrino oscillations tend

\footnotetext{
${ }^{40}$ See [243] for a recent discussion.

${ }^{41}$ The relation between these parameters is given by $\eta_{B} \simeq 2.739 \cdot 10^{-8} h^{2} \Omega_{B}$, where $\mathrm{h}$ parametrises the the Hubble rate $H_{0}=100 h(\mathrm{~km} / \mathrm{s}) / \mathrm{Mpc}$.

${ }^{42}$ A reaction "freezes out" when the temperature dependent rate at which it occurs falls below the Hubble rate $H$ of cosmic expansion. This essentially means that the density of the primordial plasma has become so low that the rate at which the participating particles meet is negligible. A particle freezes out when the last process (other than decay) that changes its comoving number density freezes out.
} 
to make the asymmetries in individual flavours equa 43 [257 259]. How complete this equilibration is depends on the mixing angle $\theta_{13}$. The measured value for $\theta_{13}$ suggests a high degree of equilibration [260]. Lepton asymmetries in the neutrino background can be constrained due to their effect on the momentum distribution, which changes the relation between temperature and

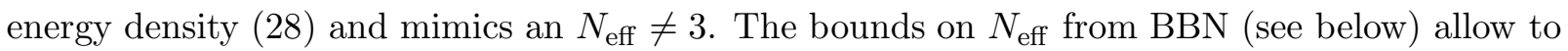
constrain the asymmetry to roughly $\left|\eta_{L}\right| \lesssim 0.1$ [260], where $\eta_{L}$ is defined analogously to (29); see also [261 264].

If sterile neutrinos are relativistic, then they can form a similar background and contribute to $N_{\text {eff. }}$. For an active-sterile mass splitting $\Delta m^{2}<1.3 \times 10^{-7} \mathrm{eV}^{2}$ active-sterile oscillations are effective only after the active neutrino freezeout, then they simply distort the momentum distributions [232]. For much larger splittings they can be produced efficiently via their mixing at $T>1 \mathrm{MeV}$. The preference for $N_{\text {eff }}>3$ in different cosmological data sets (see below) can be interpreted as a hint for $\mathrm{eV}$ mass sterile neutrinos as $\mathrm{DR}$, see section 4.2. However, the constraints on $N_{\text {eff }}$ from BBN, see below, imply that such background must either have frozen out considerably earlier if it ever was in equilibrium or never thermalized (e.g. because the production was suppressed by some mechanism).

Big bang nucleosynthesis $(T \lesssim 100 \mathrm{keV})$ - There was a brief period in the early universe during which the temperature was low enough for nuclei heavier than hydrogen $(\mathrm{H})$ to exist and still high enough for thermonuclear reactions to occur. During this period most of the deuterium (D), helium $\left({ }^{3} \mathrm{He},{ }^{4} \mathrm{He}\right.$ ) and lithium (mainly ${ }^{7} \mathrm{Li}$ ) in the universe were formed [265], see e.g. chapter 22 in [2] for a review. These light elements, in particular ${ }^{4} \mathrm{He}$, make up the vast majority of all nuclei other than $\mathrm{H}$ in the universe. Sterile neutrinos can affect this big bang nucleosynthesis (BBN) in different ways, depending on their mass. $N_{I}$ with masses far above the electroweak scale have no effect on BBN, as they have decayed long before. If the masses are in the GeV to TeV range, these particles can be long lived enough that the entropy released during their decay affects $\mathrm{BBN}$ or the thermal history afterwards. The good agreement between BBN calculations and the observed $\mathrm{H}$ and $\mathrm{He}$ abundances implies that, if sterile neutrinos with $\mathrm{GeV} \lesssim M_{I} \lesssim \mathrm{TeV}$ exist, they must have decayed sufficiently long before BBN. The resulting bounds [250, 251, 266] in the mass-mixing plane are plotted in figure 11. If $\mathrm{DM}$ is composed of $\mathrm{keV}$-mass sterile neutrinos, these would have no visible effect on $\mathrm{BBN}$ because their number density is too low to affect the expansion history in that era. Light sterile neutrinos (with eV masses) would, on the other hand, significantly contribute to $N_{\text {eff }}$ [231, 267, 268] as additional radiation and increase the rate of expansion of the universe via (27) and (28). This, for instance, determines the precise moment of neutron freeze-out $(T \sim 0.8 \mathrm{MeV})$, which roughly occurs when the expansion rate (27) equals the rate for the reaction $n+e^{+} \leftrightarrow p+\bar{\nu}_{e}$. It also affects the amount of time that passes until the formation of elements $(T \sim 10-100 \mathrm{keV})$, during which neutrons decay. Both determine the number of neutrons available for fusions [269, 270]. However, a change in the expansion rate is not specific to light neutrinos, hence the resulting change in the value for $N_{\text {eff }}$ extracted from fits to light element abundances does not actually "measure" the number of light neutrinos or other particles (see e.g. [271] for a summary of examples). It simply parametrizes any deviation from the SM prediction. Sterile neutrinos can also affect BBN if they cause deviations of the neutrino momentum distributions from a Fermi-Dirac spectrum, e.g. by active-sterile oscillations or by

\footnotetext{
${ }^{43}$ This is often referred to as "flavour equilibration", though the process occurs close to the neutrino freezeout and neutrinos may not reach thermal equilibrium. This means that the lepton asymmetry cannot be translated into a chemical potential in the strict sense.
} 
inducing chemical potentials [272]. On one hand, this slightly modifies the relation (28) between $T$ and $\rho_{\nu}$, which again affects the rate of expansion. More importantly, the He abundance is directly affected by a distortion of the $\nu_{e}$ spectrum [231]. D is less affected, hence provides a more direct probe of the expansion rate [273].

BBN depends on $\Omega_{B}, N_{\text {eff }}$ and the lepton asymmetries $Y_{\alpha}$. In the SM, where $N_{\text {eff }}=3$ is fixed and $Y_{\alpha}=0$, the only free parameter during BBN is $\Omega_{B}$ (or $\eta_{B}$ ), and there is a rather impressive agreement between theoretical predictions and the value obtained from the light elements, see e.g. [274]. If one treats $N_{\text {eff }}$ as a free parameter using (28), a deviation from 3 mainly reflects in the ${ }^{4} \mathrm{He}$ abundance. The values for $N_{\text {eff }}$ obtained from BBN alone (see e.g. [274 276], cf. also [277, 278]) show a slight preference for $N_{\text {eff }}>3$, but are consistent with $N_{\text {eff }}=3$. Different interpretation of the data are discussed in [270]: In absence of significant lepton asymmetry, BBN alone yields $N_{\text {eff }}=3.71_{-0.45}^{+0.47}$. If one fixes the He abundance to the value inferred from the CMB. this tightens to $N_{\text {eff }}=3.53_{-0.63}^{+0.66}\left(N_{\text {eff }}=3.22 \pm 0.55\right.$ when using the D abundance measured in [276] alone). The likelihood functions given in [275] show a preference for $N_{\text {eff }}>0$, but clearly constrain $N_{\text {eff }}<4$. Hence, sterile neutrinos with $\mathrm{eV}$ masses, as motivated by the oscillation anomalies (ii), can only be made consistent with BBN if they are not thermalized. This strongly constrains models of light sterile neutrinos unless some mechanism suppresses their production. If DM is composed of sterile neutrinos with $\mathrm{keV}$ masses (see section 6), these do not affect $N_{\text {eff }}$ because their number density is well below the equilibrium value (their number density can simply be obtained by putting today's $\Omega_{B}$ in relation to their mass). Hence, they are unaffected by these bounds.

Matter - radiation equality $(T \sim 0.8 \mathrm{eV})$ - As the universe expands, the matter density is diluted as $\propto a^{3}$, where $a$ is the scale factor, due to the increasing physical volume. The radiation energy density is diluted faster $\left(\propto a^{4}\right)$ due to the stretching of the wavelengths, hence an initially radiation dominated universe becomes matter dominated at the point of matter-radiation equality. In cosmology, one refers to all relativistic degrees of freedom (particle energy dominated by momentum) as "radiation", while nonrelativistic degrees of freedom (particle energy dominated by mass) are "matter". A first principles derivation of the kinetic equation in the expanding universe that covers both regimes is given in [279]. Due to Hubble expansion, constituents of the primordial plasma change their identity from "radiation" to "matter" when $T$ (more precisely: their average momentum) falls below their mass, which has to be taken into account when determining the point of equality. If sterile neutrinos are relativistic near $T \sim 1 \mathrm{eV}$, they change the temperature of matter radiation equality. This is crucial for the growth of density perturbations in the primordial plasma, see following paragraph. If they become nonrelativistic just around this time, their equation of state is neither that of "radiation" nor "matter" in the intermediate regime. The effect of the time dependence of the equation of state has e.g. been studied in [280].

Photon decoupling $(T \sim 0.25 \mathrm{eV})$ - The universe becomes transparent when the temperature is so low that photons cannot dissociate $\mathrm{H}$ atoms any mort 44 . While photons previously scattered frequently with free electrons, the cross section with neutral atoms is so small that the average

\footnotetext{
${ }^{44}$ The temperature at which this happens is much lower than the $\mathrm{H}$ binding energy because of the small baryon to photon ratio $\eta_{B}$, which implies that there are enough photons in the high energy tail of the Bose-Einstein distribution to dissociate the atoms.
} 
photon has not interacted with matter ever since. The cosmic microwave background (CMB) formed by these primordial photons allows us to observe the universe at a very early stage (redshift $z \sim 1100$ ). The CMB contains an enormous amount of information and is, altogether, one of the most impressive confirmations of the $\Lambda \mathrm{CDM}$ model. The vast amount of data also makes it an excellent tool to look for hints of physics beyond $\Lambda$ CDM and the SM. The small temperature fluctuations (of relative order $\delta T / T \sim 10^{-5}$ ) in the CMB yield the earliest probe of structure in the universe. They were generated by acoustic oscillations of the coupled baryonphoton plasma in the primordial gravitational potential wells (DM also falls into these wells, but does not feel the radiation pressure and does not oscillate).

The fluctuations can be decomposed into modes with wave numbers $k$, their spatial extension is characterized by the inverse of $k$. When $1 / k$ is larger than the causal horizon, the mode remains "frozen". After the end of inflation, density fluctuations successively "(re)enter the horizon"; that is, the causal horizon becomes bigger than their spatial extension. Then their amplitude starts to grow due to gravitational infall. The evolution in the radiation dominated era is governed by the competition between gravity and the radiation pressure (which depends on $N_{\text {eff }}$ ); this competition leads to baryon acoustic oscillations (BAO) of the plasma. The modes oscillate under the action of both forces until they decouple (when the baryons stop feeling the pressure) and start to collapse under the action of gravity, eventually forming the the structures that we observe in the universe. The observed peaks in the CMB power spectrum can be identified with multipoles $l$ in the multipole expansion of the CMB fluctuations that correspond to the modes $k$ which reached the maximal elongation at the moment of decoupling. The first peak corresponds to the smallest $k$ that reached maximal compression by the time of decoupling and so on. The evolution of perturbations can be studied quantitatively by a coupled set of Boltzmann and Einstein equations. Before photon decoupling, the density perturbations are small and $\delta_{k} \rho / \rho \ll 145$ can be used as as an expansion parameter in calculations.

The power spectrum of temperature fluctuations can be affected by $\mathrm{RH}$ neutrinos in different ways [27, 232, 281]. If they are nonrelativistic, they act as DM. Then they only affect the growth of perturbations via their free streaming length (the DM sterile neutrinos discussed in section 6 are relativistic at freezeout, but become nonrelativistic between BBN and the decoupling of photons). If they are relativistic (as e.g. the eV-mass- $N_{I}$ motivated by oscillation anomalies), they act as radiation and modify the power spectrum in several ways [281 287]. Their contribution to $N_{\text {eff }}>3$ increases the rate of expansion (27), hence reduces the comoving sound horizon, which moves the peaks in the power spectrum to higher multipoles. It also enhances the hight of the first two peaks via the integrated Sachs-Wolfe effect [283]. Light sterile neutrinos are essentially collisionless and can, in contrast to baryons and photons, not be treated as a perfect fluid. Their anisotropic stress affects the gravitational potential via Einstein's equations. Since this effect is more relevant in the radiation dominated era, it affects modes that enter the horizon before and after matter-radiation equality in a different way, which reflects in a change in the relative height of high and low $l$ peaks [285]; it suppresses the modes $l \gtrsim 200$ and also shifts the positions of the peaks [281]. Finally, sterile neutrinos contribute to the damping of small scales via their free streaming and affect the high- $l$ modes [281, 282, 288]. All these effects can be parametrized in terms of $N_{\text {eff }} 46$

\footnotetext{
${ }^{45}$ Here $\delta \rho_{k} / \rho$ collectively refers to the contrasts in the densities of photons, baryons, neutrinos and metric degrees of freedom, which of course have to be studied independently.

${ }^{46}$ Note that individually they are degenerate with other effects, cf. e.g. the discussion in [27] and references therein, and only a global fit allows to reliably determine $N_{\text {eff }}$.
} 


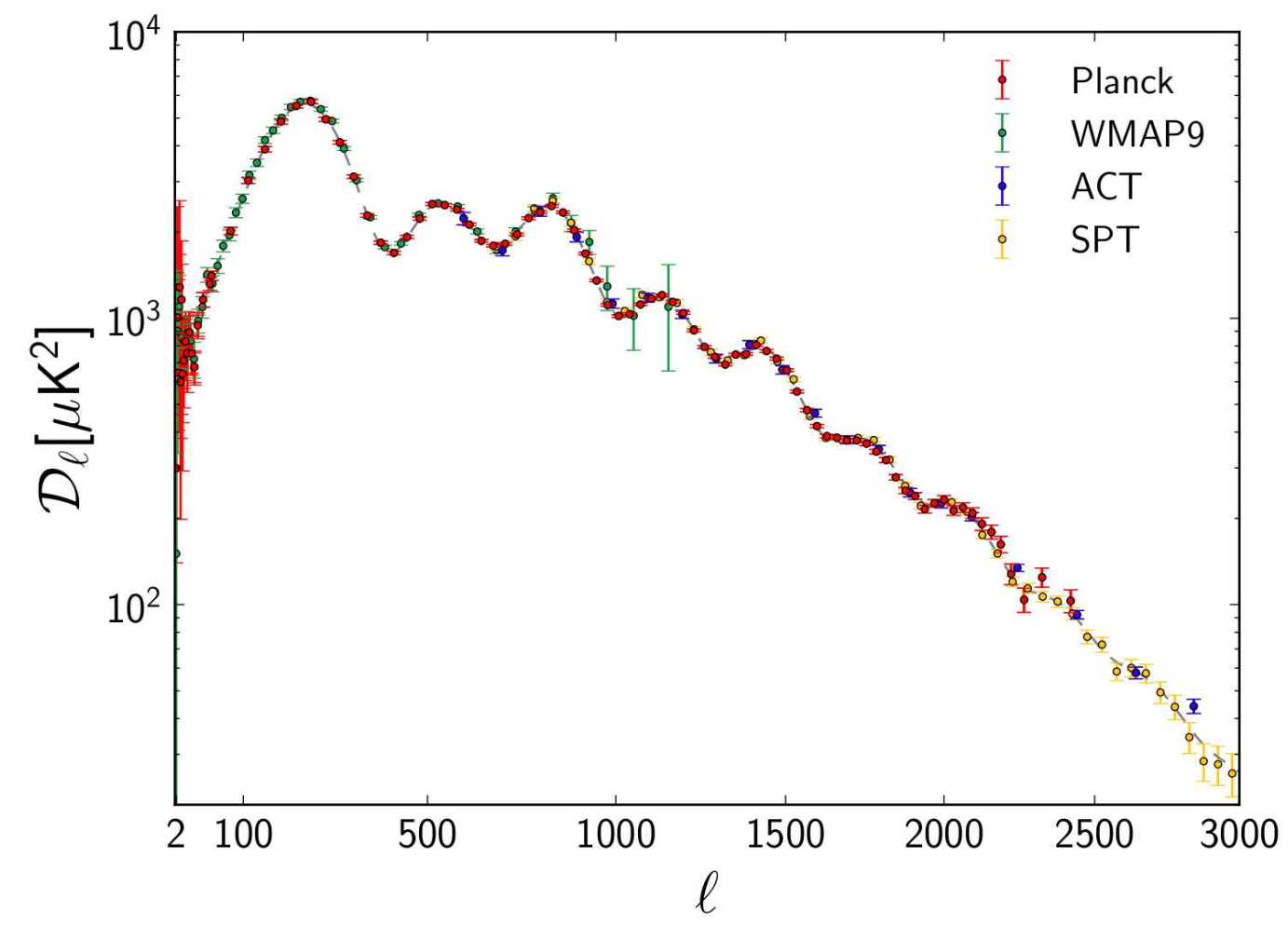

Figure 7: CMB power spectrum as measured by WMAP [237], SPT [289], ACT [290] and Planck [291]; plot taken from [291].

The CMB power spectrum has been studied with different instruments, including the Wilkinson Microwave Anisotropy Probe (WMAP) satellite, South Pole Telescope (SPT), Atacama Cosmology Telescope (ACT) and Planck satellite, see figure 7 A few years ago these datasets consistently preferred $N_{\text {eff }}>3$. In order to constrain $N_{\text {eff }}$, it is crucial to have information from high and low multipoles $l$. While WMAP with its full sky coverage can measure the low $l$ at good precision, its angular resolution is not high enough to go beyond the third peak. ACT and SPT have a better resolution, but can only observe parts of the sky and cannot go below $l \sim 500$. Thus, these data sets need to be combined to reduce the degeneracies between $N_{\text {eff }}$ and other parameters. Planck has, for the first time, measured low and high $l$ with a single instrument. Combining WMAP 7 year data with ACT in 2011 preferred $N_{\text {eff }}=5.3 \pm 1.3$ (which reduced to $4.6 \pm 0.8$ if data from BAO and measurements of the Hubble rate $H_{0}$ are added) 290], combining SPT with WMAP7 gave $N_{\text {eff }}=3.85 \pm 0.62\left(3.86 \pm 0.42\right.$ with BAO and $\left.H_{0}\right)$ [289]. More recent SPT results [292] continue to favour DR with $N_{\text {eff }}=3.62 \pm 0.48$ (3.71 \pm 0.43 when combined with $\mathrm{BAO}$ and $H_{0}$ data). In contrast to that, new ACT data [293, 294] favours $N_{\text {eff }}=2.79 \pm 0.56$, in accord with the SS. This tension between SPT and ACT has e.g. been discussed in [94, 295]. The WMAP collaboration quotes $N_{\text {eff }}=3.84 \pm 0.40$ using their 9 year data [237]. A combined analysis performed in [296] yields $N_{\text {eff }}=3.28 \pm 0.40$. Very recently, Planck found $N_{\text {eff }}=3.30 \pm 0.27$ [48], which is perfectly consistent with the SS. We discuss the implications of these measurements for sterile neutrinos as DR in section 4.2 . 


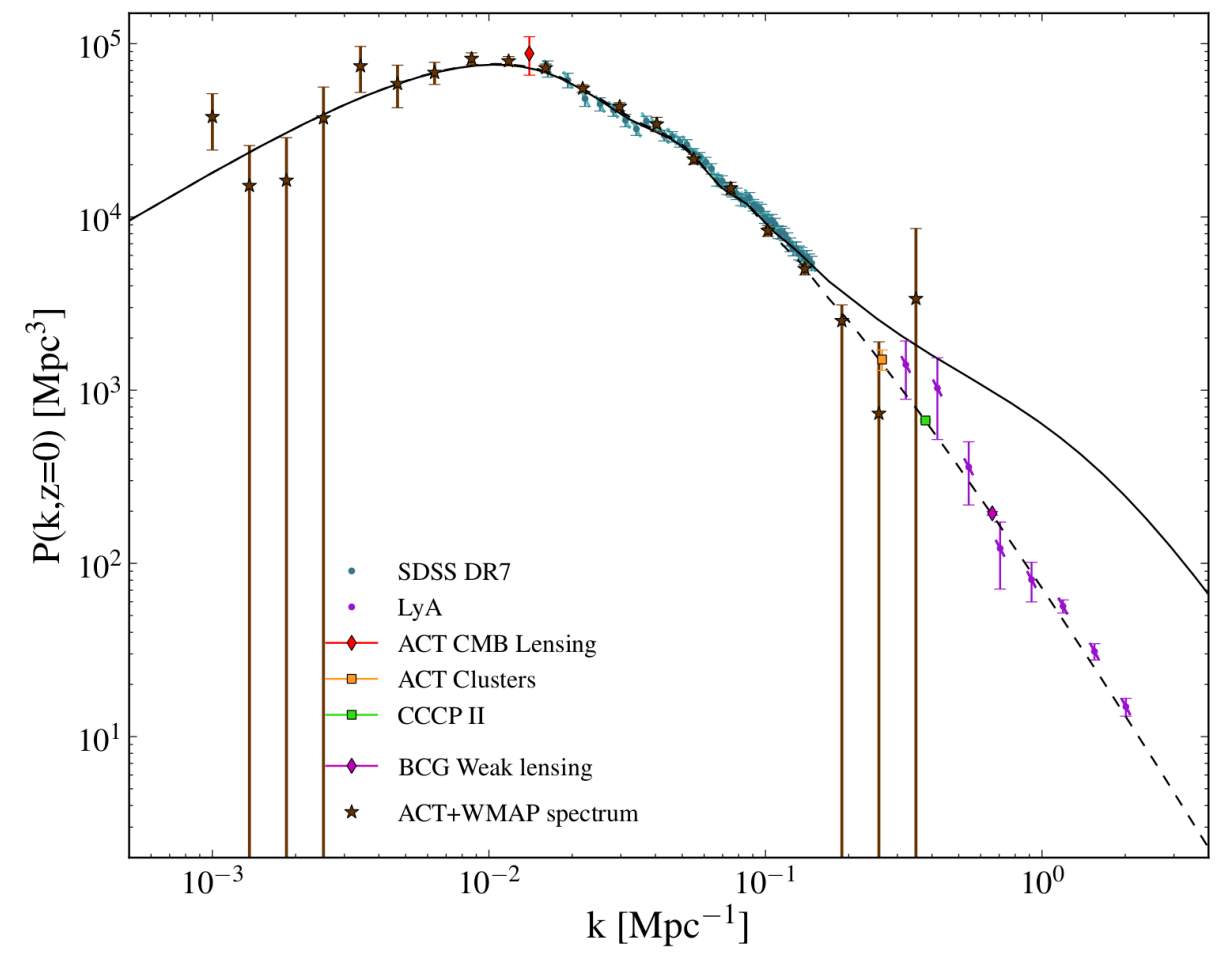

Figure 8: Matter power spectrum from different observations as indicated in the plot and combined in [297] (plot taken from there).

Large Scale Structure The formation of structures in the universe (see [298] for a review) after photon decoupling can roughly be divided into two parts. As long as $\delta \rho_{k} / \rho \ll 1$ can be used as an expansion parameter ("linear regime"), semianalytic methods [299] and fully automated codes (such as CAMB [300, 301] or CLASS48 [302]) are applicable. These can test a large number of cosmological parameter sets in a short time. For $\delta \rho_{k} / \rho>1$ ("nonlinear regime") the applicability of semianalytic methods (see e.g. [303 305]) is very limited; in general only expensive numerical n-body simulations [306 312] allow to make predictions. In the intermediate range, some sophisticated methods have been suggested (see e.g. [313 319]). For perturbations on different scales, the evolution becomes nonlinear at different times; the behaviour on cosmic scales is still linear nowadays, while the matter distribution locally is extremely inhomogeneous. Sterile neutrinos can affect the structure formation if they act as DM or DR.

The implications of $\mathrm{DM}$ sterile neutrinos with $\mathrm{keV}$ masses are discussed in section 6, the only big difference to CDM scenarios lies in their free streaming length $\lambda_{D M}$. This leads to a suppression of structures on (comoving) scales smaller than $\lambda_{D M}$. Lighter sterile neutrinos with $\sim \mathrm{eV}$ masses as suggested by the oscillation anomalies (ii) would act as DR and as hot dark matter (HDM) contribution to $\Omega_{D M}$. The effect that they have on the expansion rate (27) would not only reflect in the abundances of light elements and the CMB, but also in the distribution of

\footnotetext{
${ }^{47}$ http://camb.info

${ }^{48}$ http://lesgourg.web.cern.ch/lesgourg/class.php
} 

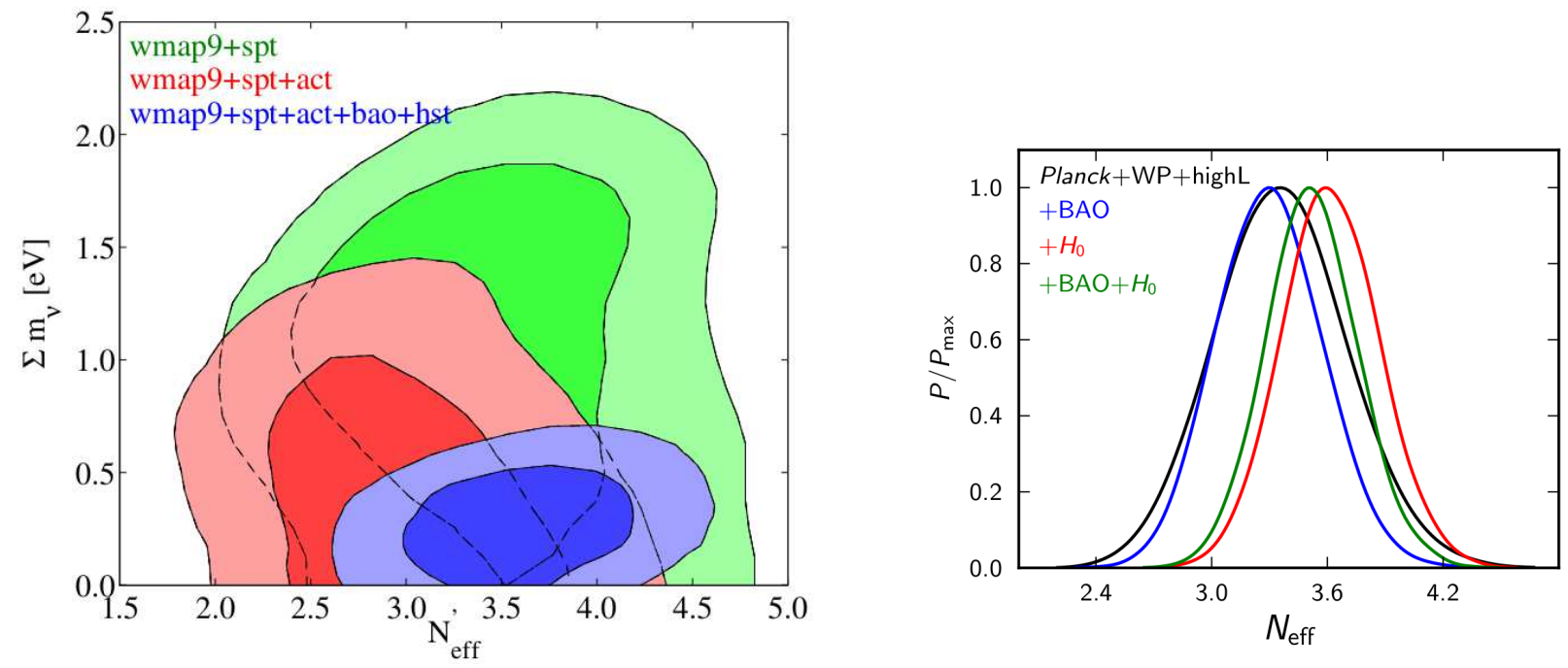

Figure 9: Left plot: Combinations of $N_{\text {eff }}$ and the sum of neutrino masses favoured by the analysis in [94]. Note that the parameter $N_{\text {eff }}$ on the horizontal axis is not exactly identical to the parameter in (28) because the authors allowed for a common neutrino mass. Right plot: Marginalized posterior distribution of $N_{\text {eff }}$ from Planck (black) and Planck data supplemented by BAO data 323, 325, 326, 328, 330] (blue), a local $H_{0}$ measurement [335] (red), and both BAO and $H_{0}$ (green); plot from [48].

matter in the universe.

An important quantity that characterizes the distribution of large scale structures (LSS) in the universe is the matter power spectrum, cf. figure 8, which is obtained from large galaxy surveys [320 330] and measures fluctuations on smaller scales than the CMB. Apart from small wiggles (related to baryonic acoustic oscillations), the matter power spectrum shows a "turnover point", the position of which is sensitive to $N_{\text {eff }}$. This feature occurs because perturbations oscillated in the radiation dominated era after they enter the horizon and grew only slowly, while in the matter dominated era they grew more quickly. The initial power spectrum is almost scale invariant, but those modes that entered the horizon during radiation domination grew only slowly until the moment of matter-radiation equality and are suppressed with respect to those that enter after that point. A larger $N_{\text {eff }}$ leads to a later equality, which affects the position of the turnover point. The damping of modes right of the turnover point is controlled by the ratio $\Omega_{B} /\left(\Omega_{B}+\Omega_{D M}\right)$. It is also affected by the velocity dispersion of massive neutrinos [27]. However, since the position of the turnover point is only known with limited accuracy [331] and LSS data cannot constrain all cosmological parameters, it is usually analysed in combination with CMB data (see below).

\subsection{Sterile neutrinos as dark radiation}

For the past decade, cosmological data generally favoured $N_{\text {eff }}>3.046$, though the significance was not strong enough to rule out the SS. It is tempting to think that sterile neutrinos may be the common origin of the oscillation anomalies (ii) and these observations (i). For the masses and mixings indicated by LSND, the thermal production of the sterile neutrinos in the early universe would be efficient enough to thermalize them [332 334], leading to a contribution to $N_{\text {eff }}$ of order one per species. 
Unfortunately, the current situation is rather confusing. On one hand there is some tension amongst different oscillation experiments [84]; most of the region favoured by LNSD is excluded by ICARUS, KARMEN and MiniBooNE. On the other hand, there is also tension between the latest results from the CMB. SPT+WMAP results [292] favour DR with $N_{\text {eff }}=3.62 \pm 0.48$; this is consistent with the $N_{\text {eff }} \simeq 4$ predicted by a thermalized sterile neutrino with masses and mixing suggested by the oscillation anomalies, but also does not rule out the SS. In contrast, ACT +WMAP [293, 294] favours $N_{\text {eff }}=2.79 \pm 0.56$, which would clearly rule out thermal DR. This is in contrast to earlier ACT results [290], which favoured DR. Planck found $3.30 \pm 0.27$ [48] (also using BAO data); though this shows a slight preference for $N_{\text {eff }}>3$, it is perfectly consistent with the SS and clearly disfavours $N_{\text {eff }}=4$.

The WMAP, ACT and SPT results have been combined with various other data sets, including measurements of the CMB, the LSS and BAO from galaxy surveys and measurements of the contemporary Hubble constant $H_{0}$. For instance, in [94] it was recently argued that cosmology can be consistent with the neutrino oscillation anomalies. This conclusion was mainly possible because the authors excluded ACT and BBN data from the combined analysis. This can be seen in figure 9. For small masses $<0.5 \mathrm{eV}$, WMAP+SPT+ACT data prefer extra neutrinos; but for masses around $1 \mathrm{eV}$ the data favours $N_{\text {eff }} \sim 3$, as predicted by the SS. Including BAO and $H_{0}$ data indicates $N_{\text {eff }}>3$, but also disfavours the $\sim 1 \mathrm{eV}$ region for the masses preferred by oscillation anomalies. Without ACT, $N=4$ and a mass of $1 \mathrm{eV}$ are perfectly consistent. Similar analyses, using pre-Planck data, have e.g. been performed in [278, 288, 295, 336 342] 49 . The conclusions differ, depending on the selected data sets and statistical method. There is a general preference for $N_{\text {eff }}>3$, but no exclusion of the SS.

However, while the authors of [94] presented arguments to exclude ACT from their analysis, the conclusions change dramatically when the Planck result 3.30 \pm 0.27 [48] is included. Though a slight preference for $N_{\text {eff }}$ so far seems to remain [344 348], the thermalized DR $\left(N_{\text {eff }} \geq 4\right)$ that the oscillation anomalies hint at is clearly disfavoured [349]. The above result assumes massless neutrinos, but does not change significantly if the sum of neutrino masses is allowed to vary $\left(N_{\text {eff }}=3.32_{-0.52}^{+0.54}\right.$ and $\sum_{I} m_{i}<0.28 \mathrm{eV}$ when combined with BAO [48]). There is some freedom to increase $N_{\text {eff }}$ if local measurements are used to determine $H_{0}$ instead of the value obtained from the Planck data itself, see figure 9. Local measurements of cosmological quantities such as the Hubble rate or the age of the universe (by studying old objects) depend less on the assumed model of cosmology and can be used complementary to high redshift data, see [350] for a recent discussion. The Planck collaboration concludes that the tension between direct $H_{0}$ measurements and the $\mathrm{CMB}$ and $\mathrm{BAO}$ data in the $\Lambda \mathrm{CDM}$ model can be relieved by $N_{\text {eff }}>3.046$, but there is no strong preference for this extension from the CMB damping tail [48]. It remains to be seen whether this tendency continues when the full Planck data is analysed, which will provide the most stringent constraint on $N_{\text {eff from a single instrument } 351 \text { ]. }}$.

The apparent tension between oscillation anomalies and the Planck and ACT results could be eased if the thermal production of light sterile neutrinos in the early universe is somehow suppressed, e.g. by a chemical potential [278, 352 354]. Quantitative studies of the flavour evolution [255, 257 259, 355 357] are required to answer the question to which degree a lepton chemical potential can prevent the thermalization [278, 334, 353, 354, 358 366].

If one, on the other hand, interprets the Planck and ACT results as indicators that there is no DR, then it remains to be understood why so many previous analyses seemed to point towards

\footnotetext{
${ }^{49} \mathrm{~A}$ nice summary of various other ways to combine different data sets can be found in [343].
} 
its existence. The possibility that the preference $N_{\text {eff }}>3.046$ is an artefact of the prior choice in Bayesian analyses has been discussed and rejected in [367]. On the other hand, the authors of 342] found that Bayesian model selection prefers the SS, despite the fact that parameter estimates for $N_{\text {eff }}$ are larger than 3.046. In [368] it has been pointed out that there is no evidence for $N_{\text {eff }}>3$ if one relaxes the assumptions on the late history of the universe suggested by $\Lambda$ CDM when analysing the CMB. Generally, there is a lot more freedom if one allows for more physics beyond the $\Lambda \mathrm{CDM}$ scenario. In [235] it has been discussed in detail that different modifications of the SM can predict values $N_{\text {eff }}>3$ or $N_{\text {eff }}<3$, irrespective of the number of neutrino degrees of freedom. However, it seems that at least the simplest scenario in which light sterile neutrinos with generic couplings are the common explanation for the oscillation anomalies and the preference for $N_{\text {eff }}>3$ is disfavoured by Planck unless more new physics is added. At the same time, it seems unlikely that the SS can be ruled out by CMB observations in foreseeable time.

\subsection{Sterile neutrinos in astrophysics}

In addition to possible signatures in high redshift observations, sterile neutrinos may also have an effect on astrophysical phenomena at present time. Their most studied role in astrophysics is that of a DM candidate, see section 4.2, but they may have effects on other phenomena. They can, for instance, affect the transport in supernova explosions if they have $\mathrm{eV}$ [369], $\mathrm{keV}$ [370, 371] or $\mathrm{GeV}$ [372] masses. Sterile neutrinos with $\mathrm{keV}$ masses can also help to explain the high rotation velocities of pulsars [373, 374].

\section{$5 \quad$ Baryogenesis via leptogenesis}

The observable universe does not contain any significant amounts of antibaryons [245], i.e. it is highly matter-antimatter asymmetric. Given our knowledge about the thermal history of the universe, today's baryon density $\Omega_{B}$ is easily explained as the remnant of a small matterantimatter asymmetry at early times, when the temperature was high enough for pair creation processes to occur faster than the Hubble rate. The baryon asymmetry in the early universe (BAU) can be estimated by the baryon-to-photon ratio at later times,

$$
\left.\left.\frac{n_{B}-n_{\bar{B}}}{n_{B}+n_{\bar{B}}}\right|_{T \gg 1 \mathrm{GeV}} \sim \frac{n_{B}-n_{\bar{B}}}{s} \sim \frac{n_{B}}{n_{\gamma}}\right|_{T \ll 1 \mathrm{GeV}} \equiv \eta_{B}
$$

Here $n_{B}$ and $n_{\bar{B}}$ are comoving number densities for baryons and anti-baryons. $\eta_{B}$ can be determined rather consistently from BBN [274] or the CMB and LSS [237],

$$
\eta_{B}^{\mathrm{BBN}}=5.80 \pm 0.27, \eta_{B}^{\mathrm{CMB}}=6.21 \pm 0.12,
$$

both in units of $10^{-10}$. A period of cosmic inflation, as suggested by the CMB, would have diluted any pre-inflationary asymmetry. Thus, the BAU must have been created dynamically afterwards. There are three conditions for the dynamical generation of a BAU ("baryogenesis"), known as Sakharov conditions [375]: Baryon number $(B)$ violation, breaking of charge $(\mathrm{C})$ as well as charge-parity (CP) symmetry and a deviation from thermal equilibrium. In principle, all of them are fulfilled in the SM: at the quantum level, baryon number is violated [247-249] at $T>T_{E W}$ by nonperturbative sphaleron processes [246], $\mathrm{C}$ and $\mathrm{CP}$ are violated by the phase in the CKM matrix [376] and the weak interaction [377, 378], and a deviation from thermal equilibrium 
is caused by Hubble expansion. However, the $\mathrm{CP}$ violation and deviation from equilibrium are both too small in the SM to explain the observed $\eta_{B}$; see [245] for a detailed account of the Sakharov conditions and baryogenesis in the SM.

RH neutrinos described by (11) can fix both of these shortcomings. As gauge singlets, they can be out of equilibrium at temperatures when all other particles are tightly coupled by gauge interactions, and their Yukawa couplings contain several unconstrained CP-violating phases. This makes baryogenesis via leptogenesis possible [379]. In this scenario a matter-antimatter asymmetry is first generated in the leptonic sector by the CP-violating interactions of the $\nu_{R}$ and then transferred to the baryonic sector by electroweak sphaleron processes 50 Sphalerons are only effective for $T>T_{E W}$. At these temperatures the Higgs field has a vanishing expectation value, hence leptogenesis operates in the symmetric phase of the SM. In this regime the fields $\nu_{L}$ behave like massless Weyl fields that can be described as the left chiral projection of a Dirac spinor [rather than the Majorana spinors (23)]. We can define the left handed lepton numbers $L_{L, \alpha}$ as the zero components of the currents $J_{L, \alpha}^{\mu} \equiv \bar{\nu}_{L, \alpha} \gamma^{\mu} \nu_{L, \alpha}+\bar{e}_{L, \alpha} \gamma^{\mu} e_{L, \alpha}$. Analogously, we define lepton numbers $L_{R, \alpha}$ from the current $J_{R, \alpha}^{\mu} \equiv \bar{e}_{R, \alpha} \gamma^{\mu} e_{R, \alpha}$ for the RH fields. The total LH lepton number is $L_{L} \equiv \sum_{\alpha} L_{L, \alpha}$, and we furthermore define the active lepton numbers $L_{\alpha} \equiv L_{L, \alpha}+L_{R, \alpha}$. For $F=0$ the $L_{\alpha}$ are exactly conserved. The $\nu_{R}$ have a Majorana mass term $M_{M}$; in general they do not carry any conserved lepton charge, and interactions with them violate the charges $L_{\alpha} 51$ However, due to the smallness of $F$ these interactions are slow, and $L_{\alpha}$ are still approximately conserved charges for which one can define a chemical potential. If there is an (exactly or approximately) conserved charge in the $\nu_{R}$-sector, we may define "lepton numbers" for the sterile neutrinos. This is always the case at temperatures $T \gg M_{I}$, when helicity changing processes are suppressed by $M_{I} / T$; then one can interpret two helicity states of $N_{I}$ as "particle" and "antiparticle" and define sterile lepton charges $L_{I}$ as the difference of their occupation numbers, cf. (39). They contribute to the RH lepton number $L_{R} \equiv \sum_{I} L_{I}+\sum_{\alpha} L_{R, \alpha}$. The total lepton number is $L \equiv L_{L}+L_{R}$. If the flavour structure of $F$ and $M_{M}$ obeys a symmetry such that there is another conserved charge (exactly or approximately), it can be more convenient to use that charge to define "lepton numbers" in the sterile sector. A particularly interesting scenario of this kind is that in which total lepton number $L$ is almost conserved [58, 76, 115 121, 382].

When one or more of the $N_{I}$ are out of equilibrium, they can generate lepton asymmetries via various processes, including decays and inverse decays, scatterings and flavour oscillations (the probability to decay/scatter/oscillate into leptons and antileptons is different at second order in $F$ due to quantum interferences between CP-violating processes). Sphalerons violate $B$ and $L$ individually, but conserve $B-L$. At $T>T_{E W}$ they transform part of the lepton asymmetry into a baryon asymmetry; if all interactions are in equilibrium it is given by $B \sim \frac{28}{79}(B-L)$ [383, 384]. $B$ is protected from washout after sphaleron freezeout around $T \sim T_{E W}$. Since sphalerons only couple to left chiral fields, they in fact only see the leptonic charge $L_{L}$ stored in these fields, which may differ from the total lepton asymmetry $L$. Thus, the BAU we observe is determined by $L_{L}$ at $T \sim T_{E W} \sim 140 \mathrm{GeV}$. The nonequilibrium condition can be fulfilled three times for each $N_{I}$ : during their production, their freezeout, and their decay.

\footnotetext{
${ }^{50}$ The term leptogenesis is often used in a wider sense, referring to all scenarios where the source of CP-violation lies in the leptonic sector. Here we only refer to those scenarios where the BAU is generated from the interactions in (11).

${ }^{51}$ For $T<T_{E W}$ the active neutrinos also receive a Majorana mass term (14) that violates lepton number. This violation is, however, suppressed by $m_{\nu} / T$. Similar arguments apply to thermal masses in the primordial plasma, which change the kinematics of quasiparticles, but respect the symmetries of the theory [380, 381].
} 


\subsection{Leptogenesis from $N_{I}$ freezeout and decay}
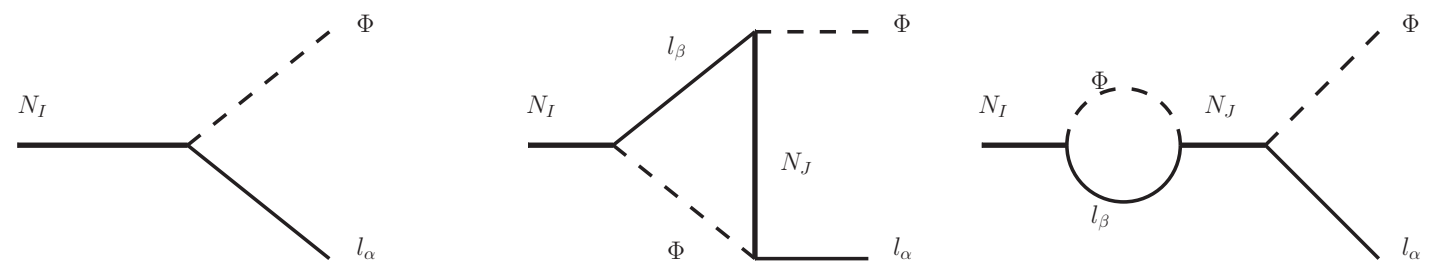

Figure 10: A crucial contribution to the CP-violation in the simplest version of "vanilla leptogenesis" comes from the quantum interference between the tree level diagram and radiative corrections to the decay $N_{I} \rightarrow \Phi l_{\alpha}$. Due to the $C P$-violation contained in the complex phases in $F$, the amplitudes for these processes with $l_{\alpha}$ in the final state differ from those with $\bar{l}_{\alpha}$. At tree level this difference amounts to an overall phase, which does not affect the physical absolute square of the amplitude, but in the interference terms between tree level and loop diagrams it makes a physical difference. The rightmost diagram gets resonantly enhanced for $M_{I} \sim M_{J}$ because then the $N_{J}$ propagator is nearly onshell. In more general scenarios, also scattering processes (such as $\Phi l_{\alpha} \rightarrow \Phi l_{\beta}$ with intermediate $N_{I}$ ) have to be considered.

It is common to split the lepton number violating processes into "source" and "washout". The source consists of all contributions that violate active lepton numbers in the absence of an existing asymmetry (e.g. $N_{I}$-decays); these can generate asymmetries in the presence of a deviation from thermal equilibrium. The "washout" consists of all other processes, which tend to eliminate existing asymmetries (e.g. inverse $N_{I}$-decays). In addition, there are "spectator processes" [385, 386] that do not violate $L$ themselves, but redistribute charges amongst different fields and thereby affect the time evolution of $L$. If the $N_{I}$ have masses as suggested by GUT models, they are produced thermally, freeze out and decay at temperatures $T \gg T_{E W}$. Since $L$ violating processes are strongly suppressed at $T<M_{I}, B-L \neq 0$ is preserved from washout. This is the most studied version of leptogenesis, and there exist several detailed reviews, see e.g. [387] or, more recently, [388, 389]. This setup is very appealing because it can easily be embedded into a GUT-framework, provides a "natural" explanation for the smallness of the neutrino masses $m_{i}$ via the seesaw mechanism and allows to probe at least some parameters of very high energy physics in low energy neutrino experiments via (10). The downside is that such heavy $N_{I}$-particles cannot be studied in the laboratory. Out of the $7 n-3$ parameters in $F$ and $M_{M}$, one can under ideal conditions probe the 3 neutrino masses and 6 angles and phases in $U_{\nu}$ experimentally (being very optimistic in case of the Majorana phases). The generated asymmetry $\eta_{B}$ (the only observable number in leptogenesis) can in general depend on all $7 n-3$ parameters. Thus, the perspectives to constrain $N_{I}$-properties from leptogenesis are very limited. We therefore only recapitulate the basic ideas and refer the interested reader to the reviews named above.

Minimal "Vanilla" leptogenesis - In the simplest scenario one assumes that the $N_{I}$ are very heavy, have hierarchical masses $\left(T_{E W} \ll M_{1} \ll M_{I>1}\right)$ and the washout is strong. The latter statement is usually parametrized in terms of the parameter $K_{I}=\left.\Gamma_{I}\right|_{T=0} /\left.H\right|_{T=M_{I}} \simeq$ $\left(F^{\dagger} F\right)_{I I} v^{2} /\left(M_{I} \times 10^{-3} \mathrm{eV}\right)$, where $\Gamma_{I}$ is the thermal width of $N_{I}$ particles, $H \simeq 1.66 \sqrt{g_{*}} T^{2} / M_{P}$ the Hubble rate and $g_{*}$ the effective number of relativistic degrees of freedom in the plasma $\left(g_{*}=106.75\right.$ in the $\mathrm{SM}$ at $\left.T \gg T_{E W}\right)$. If $\left(F^{\dagger} F\right)_{11} \sim \mathrm{eV} \times M_{1} / v^{2}$, as suggested by the seesaw relation (14), then $K_{1} \gg 1$ and one is in the strong washout regime. In this case the BAU responsible for the observed $\eta_{B}$ may have been created by the freezeout and decay of the lightest 
sterile neutrino $N_{1}$ alone. This is possible if any pre-existing asymmetries, the asymmetries generated during the production of the $N_{I}$ and the asymmetries from the $N_{I>1}$-decays are all washed out efficiently by $N_{1} 52$. This scenario has the advantage that $\eta_{B}$ is essentially independent of the initial conditions. A major contribution to the final asymmetry comes from the decay diagrams shown in figure 10. In addition, there are lepton number violating scatterings. However, vanilla leptogenesis can be qualitatively understood by considering the decays and inverse decays only [390]. This mechanism can reproduce $\eta_{B}$ for $M_{1} \gtrsim 4 \times 10^{8} \mathrm{GeV}$ [391], which requires a rather large reheating temperature if $N_{I}$ are mainly produced thermally ("thermal leptogenesis"). There is also a constraint on the mass of the lightest active neutrino as $m_{1} \lesssim 0.1 \mathrm{eV}[392$, 393]; see 394] for an review on the connection with neutrino masses. In the weak washout regime $K_{1} \lesssim 153$ the predictive power is much smaller because asymmetries from processes at earlier time (nonthermal production during reheating, thermal $N_{I}$ production, $N_{I>1}$ freezeout and decay...) are not washed out efficiently and contribute to $\eta_{B}$.

Flavour effects - In the simplest scenario it is assumed that only one sterile neutrino $N_{1}$ dynamically participates in leptogenesis and all three active flavours can be treated equivalently ("unflavoured regime"). In the unflavoured regime, only the linear combination $\sim F_{\alpha 1}\left|l_{L \alpha}\right\rangle$ of active leptons that couples to $N_{1}$ is relevant for leptogenesis. In the corresponding flavour basis one can ignore the directions in flavour space perpendicular to that, and the problem is equivalent to the one flavour case. Both of these assumptions do not hold in general.

Active flavours have to be treated separately when the Hubble rate drops below the rate at which interactions that distinguish active flavours, mediated by the charged Yukawa couplings, occur ("flavoured regime"). These interactions destroy the coherence of the flavour state that couples to $N_{1}$ because they have different strength for the different $l_{L, \alpha}$. For the $\tau$-Yukawa, this happens below $T \sim 10^{12} \mathrm{GeV}$. The importance of flavour has been realized in [395] and [396 399] and meanwhile been studied by various authors, see [388, 389] for details and references. Once two flavour states are distinguishable, one has to treat the asymmetries stored in each individually. They can differ considerable and even have opposite signs [400]. This can affect $\eta_{B}$ if e.g. the washout is very different for different flavours; it makes leptogenesis possible even if the source term does not violate $\sum_{\alpha} L_{\alpha}$. Flavour effects can reduce the lower bound on $M_{1}$ in generic seesaw scenarios by $1-2$ orders of magnitude [401, 402], which is still far out of experimental reach.

Also the assumption that the generation of today's BAU only involved $N_{1}$-dynamics does not hold in general. In the unflavoured regime, $N_{1}$ can only wash out the asymmetry that is stored in the combination $F_{\alpha 1} Y_{\alpha}$ in flavour space to which it couples. This singles out a particular direction in flavour space. If pre-existing asymmetries or those produced by $N_{I>1}$ have a component orthogonal to this, $N_{1}$ cannot wash them out efficiently [403, 404]. In fact, it is a rather special case that there is no such component. The effect of active flavours in scenarios where the heavier $N_{I}$ contribute has been discussed in [405-409].

Flavour effects offer ways to circumvent the lower bound on $M_{I}$. One possibility is that the BAU originates from a purely flavoured asymmetry; i.e. the total asymmetry is vanishing or small $(L \simeq 0)$, but the left handed asymmetry is non-vanishing $\left(\left|L_{L}\right| \gg|L|\right)[121,402]$. Since sphalerons only couple to LH fields, they convert part of $L_{L}$ into a LH baryon asymmetry. This is possible for $M_{I}<v$ because in this case the seesaw relation (14) enforces Yukawa couplings

\footnotetext{
${ }^{52}$ Though there are no $N_{I>1}$ particles in the plasma at $T \ll M_{I>1}$, the existence of more than one RH neutrino is crucial to provide $\mathrm{CP}$-violation in the lepton sector.

${ }^{53}$ This is possible in spite of (14) because $M_{M}$ and $F$ are matrices.
} 
that are so small that $L_{L}$ and $L_{R}$ may not equilibrate before sphaleron freezeout. This makes leptogenesis possible even for $M_{M}=0$ [382] (Dirac leptogenesis), and it is also the basis of scenarios discussed in the following section 5.2. The mass bound may also be circumvented if the generated asymmetry is resonantly enhanced by a degeneracy between sterile neutrino masses [410] (resonant leptogenesis). This enhancement is discussed from first principles in [411, 412]; in [411] it is shown that the maximal enhancement (compared to the non-resonant case) of the total asymmetry $L$ can be expressed in terms of the parameter 54

$$
\frac{M_{1} M_{2}}{2\left(M_{1} \Gamma_{1}+M_{2} \Gamma_{2}\right)} \text {. }
$$

The enhancement allows values of $M_{M}$ in the TeV range, which raises hope to probe $N_{I}$ in high energy experiments. In this case it is of course not only the lightest sterile neutrino $N_{1}$ that generates the asymmetry, but the interplay between the two mass-degenerate states, and flavour effects in the sterile sector have to be taken into account. This is generally the case when the spectrum of $M_{I}$ is not hierarchical $\left(\left|M_{1}-M_{2}\right| \lesssim M_{1}\right)$, not only in the extreme case $M_{1} \simeq M_{2}$.

This discussion and the quoted mass bounds apply to leptogenesis within the framework of a pure type-I seesaw. If one introduces new degrees of freedom beyond those in (1), these bounds can weaken, see e.g. [223, 418, 419], [420] and [421].

\subsection{Leptogenesis during $N_{I}$ production}

If some masses $M_{I}$ are near the electroweak scale or below, the seesaw relation (14) requires the entries $F_{\alpha I}$ to be very small to be consistent with bounds on the active neutrino masses. That means that the rate $\Gamma_{I} \sim\left(F^{\dagger} F\right)_{I I} T$ of thermal $N_{I}$ production is so small that these particles may not come into thermal equilibrium until $T \sim T_{E W}$. During the thermal production before equilibration, the nonequilibrium condition is fulfilled and asymmetries $L_{\alpha}$ are generated. The total asymmetry $L$ is suppressed by $M_{I} / T$ unless there is a resonant enhancement à la (31), but the flavoured asymmetries $L_{\alpha}$ are unsuppressed [422], hence $\left|L_{L}\right| \gg|L|$ for $M_{I} \ll T_{E W}$. This is sufficient because sphalerons only see $L_{L} \simeq-L_{R}$. In contrast to the scenarios with $M_{I} \gg T_{E W}$ discussed in section [5.1, lepton number is not conserved near $T_{E W}$ because the sterile neutrinos are still present in the plasma; once the $N_{I}$ reach thermal equilibrium, the $L_{\alpha}$ are washed out. A baryon asymmetry can be generated from $L_{L}$ in spite of this (and the smallness of $|L|$ ) if the $N_{I}$ have not thermalized or the washout is not efficient enough to eliminate $L_{L}$ before sphaleron freezeout. This scenario is often referred to as baryogenesis via neutrino oscillations [423], though individual oscillations are not always crucial [422, 424, 425]. One advantage of this mechanism is that the $N_{I}$ are light enough to be within reach of laboratory experiments. This makes it one of the few known models of baryogenesis that are empirically testable (the other two much studied testable mechanisms are resonant leptogenesis and electroweak baryogenesis [426]). The perspectives to study the $N_{I}$ responsible for baryogenesis in the laboratory have been studied in detail in [250, 251, 422, 424]. If the $N_{I}$ interact only via the Yukawa couplings $F$, then a clear detection is realistically only possible for masses $M_{I} \lesssim$ a few $\mathrm{GeV}$ (unless $F$ has some special structure, leading to cancellations in (14) that allow for larger individual elements $F_{\alpha I}$ [118, 119]). If they have additional interactions, masses up to a few $\mathrm{TeV}$ are in reach of high energy experiments, see section 3.4 and references therein for more details. In the minimal scenario with

\footnotetext{
${ }^{54}$ See [410, 411, 413-417] for earlier discussions of this point.
} 

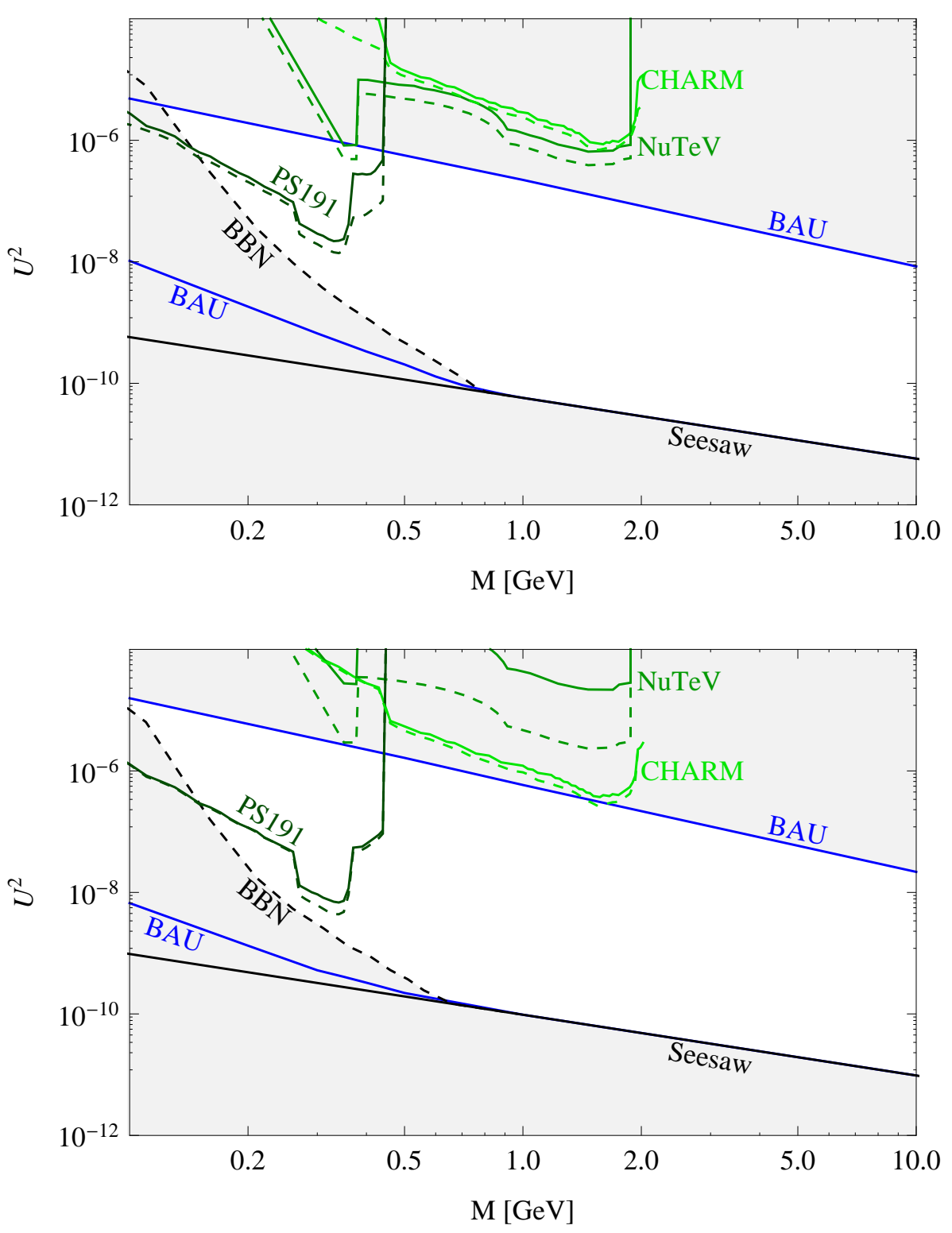

Figure 11: Constraints on the sterile neutrino masses and mixing $U^{2}=\operatorname{tr}\left(\theta^{\dagger} \theta\right)$ for $n=2$ from baryogenesis; upper panel - normal hierarchy, lower panel - inverted hierarchy. For the displayed case of $n=2 \mathrm{RH}$ neutrinos, baryogenesis can only be successful if their masses $M_{1}$ and $M_{2}$ are degenerate $\left(\left|M_{1}-M_{2}\right| \ll\left(M_{1}+M_{2}\right) / 2\right)$, the parameter $M$ refers to their mean value $\left(M_{1}+M_{2}\right) / 2$. For $n \geq 3 R H$ neutrinos no mass degeneracy is required [422]. The observed BAU can be generated in the region between the solid blue "BAU" lines. The regions below the solid black "seesaw" line and dashed black "BBN" line are excluded by neutrino oscillation experiments and BBN, respectively. The areas above the green lines of different shade are excluded by direct search experiments, as indicated in the plot. The solid lines are exclusion plots for all choices of parameters, for the dashed lines the phases were chosen to maximize the asymmetry, consistent with the blue lines. Plots taken from [251].

$n=2$ sterile neutrinos a mass degeneracy $\left|M_{1}-M_{2}\right| /\left(M_{1}+M_{2}\right) \sim 10^{-3}$ is required to produce the observed BAU (or $\eta_{B}$ ). Bounds on mass and mixing from the baryogenesis requirement in 
this case are shown in figure 11 together with other experimental and astrophysical constraints. For $n>2$ and $M_{I}$ in the GeV range no mass degeneracy is necessary [422].

The number of possible leptogenesis scenarios increases greatly when (11) is extended by additional degrees of freedom or embedded into a bigger framework (e.g. supersymmetry); we do not discuss these here in detail and refer the interested reader to the reviews [387 389].

Finally, the CP- and B-violation that make leptogenesis possible can also "work backwards" and rule out or constrain other baryogenesis scenarios because they wash out matter-antimatter asymmetries created by those mechanisms at higher temperatures. The mass spectrum of the $N_{I}$ can be constrained by the requirement that they do not wash out the baryon asymmetry in the early universe [427], though these conclusions rely on assumptions about additional interactions of $N_{I}$. A similar argument was suggested in [428] to constrain the properties of a fourth neutrino generation.

\subsection{Towards a quantitative treatment}

Transport equations - Most quantitative studies of leptogenesis solve a set of momentum integrated Boltzmann equations ("rate equations") to predict $\eta_{B}$ as a function of the parameters in (11). In the case of "vanilla leptogenesis", these can be written as [390]

$$
\begin{aligned}
H X \frac{d Y_{1}}{d X} & =-\Gamma_{1}\left(Y_{1}-Y_{1}^{e q}\right), \\
H X \frac{d Y_{B-L}}{d X} & =\epsilon_{1} \Gamma_{1}\left(Y_{1}-Y_{1}^{e q}\right)-c_{W} \Gamma_{1} Y_{B-L} .
\end{aligned}
$$

Here $Y_{1}$ is the abundance of $N_{1}$ particles, i.e. the momentum integral over the phase space distribution function divided by the entropy density $s . Y_{1}^{e q}$ is its value in thermal equilibrium and $Y_{B-L}$ the difference between baryon and lepton abundances (particles minus antiparticles for each of them). We use the variable $X=M_{1} / T$ instead of time, which leads to a factor $d X / d t \simeq H X$ on the left hand side55. $\epsilon_{1}$ is a parameter that characterizes the amount of CPviolation,

$$
\epsilon_{1} \simeq-\frac{3}{16 \pi} \frac{1}{\left(F^{\dagger} F\right)_{11}} \sum_{I} \operatorname{Im}\left[\left(F^{\dagger} F\right)_{I 1}^{2}\right] \frac{M_{1}}{M_{I}} \simeq \frac{3}{16 \pi} \frac{M_{1}}{\left(F^{\dagger} F\right)_{11} v^{2}} \operatorname{Im}\left[\left(F^{T} m_{\nu} F\right)_{11}\right]
$$

The constant $c_{W}$ in the simplest case (washout by inverse decays) is given by $1 / 2$ times the ratio between the number densities of $N_{1}$ and active leptons. Calculation of the final $Y_{B-L}$ requires solving the rate equations (32) and (33), it can be estimated as $Y_{B-L} \sim \kappa \epsilon_{1} / g_{*}[379]$ where the number $\kappa<1$ is called efficiency factor and is related to the washout.

Equations (32) and (33) can be used when only one sterile neutrino dynamically contributes to the BAU and the SM leptons can be described in an effective one flavour treatment (unflavoured regime). In the fully flavoured regime flavour dependent interactions act sufficiently fast (compared to the time scale $\sim 1 / \Gamma_{1}$ related to the $N_{1}$ dynamics and $1 / H$ ) to fully decohere the different contributions in the state $F_{\alpha 1}\left|l_{L \alpha}\right\rangle$ that couples to $N_{1}$. Then (33) has to be replaced by three different equations for the asymmetries $Y_{\alpha}$ in the individual flavours. In the intermediate

\footnotetext{
${ }^{55}$ The expression for $d X / d t$ can be complicated when $g_{*}$ changes during leptogenesis. This can e.g. affect the generation of lepton asymmetries at $T \ll T_{E W}$.

${ }^{56}$ Our notation is more close to that in 387 ].
} 
regime between these two cases, there is no full decoherence. In this case flavour oscillations have to be taken into account. This is usually done by density matrix equations [429], see [395, 398] for early applications to leptogenesis. These are matrix valued generalizations of the rate equations (32) and (33) in which the lepton charges $Y_{\alpha \beta}$ carry two indices. The diagonal elements $Y_{\alpha \alpha}$ of these are simply describe the lepton abundance in flavour $\alpha$, the off-diagonal components describe correlations between different flavours. A first principles approach that allows to study the flavoured, unflavoured and intermediate regimes consistently was presented in [430].

If more than one RH neutrino is relevant for leptogenesis, also the different sterile flavours have to be treated independently. For a hierarchical mass spectrum, this usually amounts to simply replacing (32) by $n$ different equations for the abundances $Y_{I}$ of all relevant sterile flavours $N_{I}$. In scenarios of resonant leptogenesis or leptogenesis during $N_{I}$ production, oscillations between the different flavours may be relevant. In these scenarios, leptogenesis typically happens at temperatures $T \gg M_{I}$. In this situation transitions between the different helicity states are strongly suppressed and the different helicity states of $N_{I}$ evolve independently. Effectively, they act as "particle" and "antiparticle" for the $N_{I}$, though this notion can of course not be taken literally for a Majorana field 57 . Correlations between different helicities are usually negligible, and it is justified to describe the $N_{I}$ by two matrices $Y_{N}$ and $Y_{\bar{N}}$ for the two helicity states. In the mass basis, the total abundances $Y_{I}$ can be identified with the elements $\left(Y_{N}\right)_{I I}+\left(Y_{\bar{N}}\right)_{I I}$, while the "lepton asymmetry" $L_{I}$ stored in the sterile flavour $N_{I}$ is proportional to $\left(Y_{N}\right)_{I I}-\left(Y_{\bar{N}}\right)_{I I}$. In the fully flavoured regime, where leptogenesis during $N_{I}$ production and resonant leptogenesis usually take place, the kinetic equations then read

$$
\begin{aligned}
i H X \frac{d Y_{N}}{d X} & =\left[H_{N}, Y_{N}\right]-\frac{i}{2}\left\{\Gamma_{N}, Y_{N}-Y_{N}^{e q}\right\}+\frac{i}{2} Y_{\alpha} \tilde{\Gamma}_{N}^{\alpha} \\
i H X \frac{d Y_{\bar{N}}}{d X} & =\left[H_{N}^{*}, Y_{\bar{N}}\right]-\frac{i}{2}\left\{\Gamma_{N}^{*}, Y_{\bar{N}}-Y_{\bar{N}}^{e q}\right\}-\frac{i}{2} Y_{\alpha} \tilde{\Gamma}_{N}^{\alpha *} \\
i H X \frac{d Y_{\alpha}}{d X} & =-i \Gamma_{L}^{\alpha} Y_{\alpha}+i \operatorname{tr}\left[\tilde{\Gamma}_{L}^{\alpha}\left(Y_{N}-Y_{N}^{e q}\right)\right]-i \operatorname{tr}\left[\tilde{\Gamma}_{L}^{\alpha *}\left(Y_{\bar{N}}-Y_{\bar{N}}^{e q}\right)\right] .
\end{aligned}
$$

The flavour-matrix $H_{N}$ is the dispersive part of the effective $N_{I}$-Hamiltonian, which leads to sterile neutrino oscillations; the rate-matrices $\Gamma_{N}, \tilde{\Gamma}_{N}$ and $\Gamma_{L}^{\alpha}$ form the dissipative part, which acts as collision term. These transport coefficients have to be computed from the real- and imaginary parts of the $N_{I}$ and $l_{L, \alpha}$ self energies in thermal field theory. More precise definitions are given in [251], along with a derivation of (35)-(37).

The equations (32)-(37) are rate equations for momentum integrated abundances. They provide a good approximation if the momentum distributions are proportional for equilibrium distributions (this is often called kinetic equilibrium). If this is not the case, each momentum mode has to be tracked independently by a Boltzmann equation, see e.g. [431, 432]. In numerical simulations, one of course has to sample a finite number of representative momenta. In addition, one needs to calculate the collision term as a function of momentum and temperature. This makes the treatment technically much more challenging.

Conceptual issues - A question that has received much attention in recent years is whether Boltzmann equations for particle abundances are in principle suitable to describe leptogenesis. For instance, early calculations based on Boltzmann equations were plagued with a double-counting

\footnotetext{
${ }^{57}$ At $T \gg M_{I}$ the average momentum of particles is so large that the Majorana mass is kinematically negligible, hence $\nu_{R}$ effectively behaves like at massless Weyl field up to corrections $\mathcal{O}\left[M_{M} / T\right]$.
} 
problem [433]. This problem arises if one "naively" plugs vacuum S-matrix elements as collision terms into the classical Boltzmann equations by hand. Doing so, one somewhat artificially distinguishes the external and internal lines of a Feynman diagram; only the external lines are associated with physical particles that appear in the phase space distribution functions. If one, in addition to the $N_{I}$ decays and inverse decays shown in figure 10, introduces collision terms for $N_{I}$-mediated scatterings in this way, then one counts the same processes twice: If the intermediate $N_{I}$ in a scattering is on-shell, then the scattering $l_{\alpha} \Phi \rightarrow \Phi l_{\beta}$ is identical to an inverse decay $l_{\alpha} \Phi \rightarrow N_{I}$ followed by a decay $N_{I} \rightarrow \Phi l_{\beta}$ and should not be counted independently 58 This particular problem can be fixed by hand within the Boltzmann approach by performing a real intermediate state subtraction (RIS) and using finite temperature field theory to calculate the amplitudes 59 , but it reflects a deep conceptual issue of Boltzmann equations in leptogenesis.

In leptogenesis, the leading order processes that contribute to the generation of a matterantimatter asymmetry come from an interference with loop diagrams, as e.g. shown in figure 10. hence leptogenesis is a pure quantum effect. This is in contrast to many other processes that are well-described by Boltzmann equations, such as CMB decoupling or BBN. Boltzmann equations are semi-classical. The dynamical quantities are phase space distribution functions for particles and antiparticles, i.e. classical quantities. The collision terms, on the other hand, are calculated from S-matrix elements. This treatment is based on a number of assumptions that may be questionable in the dense primordial plasma. The definition of particle numbers and the S-matrix are both based on the notion of asymptotic states, the meaning of which is not clear in a dense plasma, where particles are never "far away" from their neighbours. Even if the plasma can be described as an ensemble of (quasi)particles, the dispersion relations of these differ considerably from those in vacuum. Finally, the collision terms are affected by thermodynamic effects (such as Bose enhancement, Pauli blocking, Landau-Pomeranchuk-Migdal effect, possible enhancements from multiple scatterings...) and cannot be calculated from the (vacuum) S-matrix. The range of validity of the Boltzmann equations and size of possible corrections cannot be estimated within this framework and requires a first principles treatment. This has lead to a great interest in nonequilibrium quantum field theory [279, 439 443] and applications to leptogenesis [224, 279, 411, 412, 419, 430, 438, 444 458] in recent years.

At a fundamental level, the state of any quantum system can be described by an infinite tower of n-point correlation functions. In practice, it is usually sufficient to consider two-point functions. The leptonic charge can conveniently be described in terms of a correlation function known as statistical propagator $60 S_{L}^{+}\left(x_{1}, x_{2}\right)_{\alpha \beta}=\frac{1}{2}\left\langle l_{L, \alpha}\left(x_{1}\right) \overline{l_{L, \beta}}\left(x_{2}\right)-\overline{l_{L, \beta}}\left(x_{2}\right)^{T} l_{L, \alpha}\left(x_{1}\right)^{T}\right\rangle$, where the transposition applies to spinor indices (which we have suppressed here). The average $\langle\ldots\rangle=$ $\operatorname{Tr}(\varrho \ldots$ ) includes quantum mechanical and thermodynamic fluctuations ( $\varrho$ is the density operator $[459,460])$. The definition of this two-point function does not depend on any notion of asymptotic states or (quasi)particles. From the Fourier transform of the statistical propagator in the relative

\footnotetext{
${ }^{58}$ This side of the problem is related to the bookkeeping of physically different processes in the plasma. In addition, the use of vacuum S-matrix elements neglects finite density corrections to the propagators. The incomplete inclusion of quantum statistics can lead to an overestimate of the generated asymmetry, which has happened repeatedly in the literature. Most recently this was pointed out for the soft leptogenesis [434 436] mechanism in [419]. Using a more complete treatment, the authors there conclude that soft leptogenesis [437] cannot explain $\eta_{B}$ for generic parameter choices.

${ }^{59}$ See [438] and references therein for a detailed account of this issue.

${ }^{60}$ Here we use the notation of [452].
} 
coordinate $x_{1}-x_{2}$ one can define the momentum integrated lepton abundance matrices

$$
Y_{l}(t) \equiv-\int \frac{d^{4} p}{(2 \pi)^{4}} \frac{\operatorname{tr}\left[\gamma^{0} S_{L}^{+}(p ; t)\right]}{s}=-\int d \mathbf{p} \frac{\mathbf{p}^{2}}{2 \pi^{2}} \int \frac{d p_{0}}{2 \pi} \frac{\operatorname{tr}\left[\gamma^{0} S_{L}^{+}(p ; t)\right]}{s} .
$$

Here $x_{i}=\left(t_{i}, \mathbf{x}_{i}\right), t=\left(t_{1}+t_{2}\right) / 261$ and the trace runs over Dirac indices. The diagonal elements $Y_{l}(t)_{\alpha \alpha}$ correspond the lepton numbers in flavour $\alpha$, the off-diagonals describe correlations between different flavours during flavour oscillations. For the sterile neutrinos we define the statistical propagator $G_{I J}^{+}\left(x_{1}, x_{2}\right)=\frac{1}{2}\left\langle N_{I}\left(x_{1}\right) \bar{N}_{J}\left(x_{2}\right)-\bar{N}_{J}\left(x_{2}\right)^{T} N_{I}\left(x_{1}\right)^{T}\right\rangle$. The matrices $Y_{N}$ and $Y_{\bar{N}}$ can be extracted from it as

$$
Y_{N}(t) \equiv-\int \frac{d^{4} p}{(2 \pi)^{4}} \frac{\operatorname{tr}\left[\mathrm{P}_{+} \gamma^{0} G^{+}(p ; t)\right]}{s}, Y_{\bar{N}}(t) \equiv-\int \frac{d^{4} p}{(2 \pi)^{4}} \frac{\operatorname{tr}\left[\mathrm{P}_{-} \gamma^{0} G^{+}(p ; t)\right]}{s}
$$

where $\mathrm{P}_{ \pm}$are helicity projectors. The time evolution of $G^{+}$and $S_{L}^{+}$is governed by the KadanoffBaym equations [461], which can be obtained from a Dyson-Schwinger equation on a complex time path [462 465]. The Kadanoff-Baym equations are exact integro-differential equations; There are different ways to derive effective kinetic equations for $Y_{N}, Y_{\bar{N}}$ and $Y_{l}$ from them [279, 412, 430, 438, 452, 456]. In the fully flavoured or unflavoured regime, one can neglect active flavour oscillations and use a flavour basis in which $Y_{l}$ is diagonal. In this case we can, assuming that sphalerons act rapidly on the time scale related to the $N_{I}$-evolution with a rate that is in good approximation given by the equilibrium one 62 , relate $Y_{l}$ to the quantities in (35)-(37) as $Y_{\alpha}=\left(Y_{l}\right)_{\alpha \alpha}-\left(n_{B}-n_{\bar{B}}\right) / 3$.

Though the above approach provides a controlled approximation scheme to formally obtain effective kinetic equations from first principles, it is not yet a complete theory of leptogenesis because the computation of the transport coefficients in practice can be complicated 225, 253, 468 475]. It requires knowledge of the quasiparticle spectrum in the plasma [223, 412, 456, 476479] and inclusion of all processes in the plasma [223, 224, 471, 473]. In the regime $M_{I} \ll T$ it is still not clear which effect corrections from soft and collinear gauge boson exchange have [225, 471 474].

The conclusion on this point is that it is possible to describe leptogenesis in the unflavoured [449, 452], flavoured [430, 451] and resonant [411, 412] regimes by effective kinetic equations. By that, we mean differential equations that are local and of first order in time [279], which is in contrast to the non-local second order fundamental Kadanoff-Baym equations. The situation is relatively simple if the lepton asymmetry is generated at $T \ll M_{I}$ and the $M_{I}$ spectrum is hierarchical. Then first principles calculations suggest that in the unflavoured [449, 452] and fully flavoured [430] cases standard Boltzmann equations give results which are correct up to factors $\mathcal{O}[1]$, provided that the RIS is performed consistently. When flavour oscillations are relevant, one has to use their matrix-valued generalisation, the density matrix equations, as e.g. derived in [251, 430, 456]. For $T>M$ or when two masses are degenerate, one can still formulate effective kinetic equations, such as (35)-(37). However, inserting S-matrix elements into the classical Boltzmann equation may not give a correct quantitative description. Instead, they should be derived using controlled approximations to the full nonequilibrium field theory, which take into account the modified (quasi)particle spectrum and dispersion relations in the plasma, the effect of (possibly multiple) scatterings with quanta from the thermal bath and other thermodynamic effects. Though great progress has been made in this regime [225, 251, 411, 412, 422, 450, 471473, 480], the accurate quantitative description is not complete at this stage.

\footnotetext{
${ }^{61}$ In the homogeneous and isotropic early universe there is no dependence on $\mathbf{x}_{1}+\mathbf{x}_{2}$.

${ }^{62}$ See [466, 467] for some discussion of this point.
} 


\section{Sterile neutrinos as dark matter}

Over the past 80 year 63 , overwhelming evidence has accumulated that most of the mass in the observable universe is not composed of baryonic matter64 as we know it. The presence of large amounts of non-luminous matter can on one hand be inferred by comparing the observed gravitational potentia 65 to the density of visible matter. Independently of that, it is also required in order to explain the clustering of matter and formation of structures in the universe, e.g. the power spectrum shown in figure 8 . This process can be studied by the analysis of density perturbations in the $\mathrm{CMB}^{66}$ and by observations of the structure in the universe, such as galaxy surveys, gravitational lensing or the absorption in the spectra of distant quasars ("Ly $\alpha$ forest").

Numerous attempts to simultaneously explain these phenomena by modifications of gravity or the presence of compact macroscopic objects (such as lonely planets, black holes or other non-luminous star remnants) have failed. In contrast to that, they can easily be understood if one assumes the existence of (one or several) new particles that are massive, electrically neutral, long lived (compared to the age of the universe), collisionless and have a free streaming length in the matter dominated era that is sufficiently small to be consistent with the observed structure in the universe.

One can qualitatively distinguish three types of DM candidates. cold dark matter (CDM) is composed of particles that were nonrelativistic at the time of decoupling. hot dark matter (HDM) particles were relativistic at the time of decoupling and remain so into the matter dominated epoch, when structures can grow nonlinearly due to gravitational collapse. warm dark matter (WDM) is relativistic at the time of freezeout, but becomes nonrelativistic during the radiation dominated epoch.

CDM scenarios predict that smaller scale objects form first and then merge into bigger structures ("bottom up") [483, 484]. This is in good agreement with observations as well as numerical simulations of LSS. On smaller scales, it was noticed more than a decade ago [485, 486] and remains an unsolved puzzle that CDM simulations tend to predict more objects (satellite galaxies and subhalos) than observed. Furthermore, they do not reproduce shape of galactic halos ("cusp/core issue"). However, at this stage this is far from being a reason to disregard CDM because the discrepancy may be related to the resolution or other technical limitations of the simulations (to date, most of them are pure DM simulations that do not include baryons consistently). Furthermore, small objects are hard to observe if they fail to confine gas and form stars, see e.g. [487 490], though this may not explain the discrepancy for objects that are too big to fail to attract gas, see e.g. [491]. A recent summary on issues and possible solutions in the $\Lambda$ CDM model can be found in [492].

In contrast to that, HDM predicts that large structures form first 299] because primordial fluctuations in the gravitational potential have been erased due to the large free streaming length

\footnotetext{
${ }^{63}$ The term "dark matter" was already used in [481, 482], but in that context simply referred to non-luminous matter. Zwicky concluded that the velocities in the Coma-system are an "unsolved problem", which, however, did not receive much attention for decades.

${ }^{64}$ In this context all matter that is composed of SM fermions (including leptons) is usually referred to as "baryonic".

${ }^{65}$ The gravitational potential can be studied by tracking the dynamics of astrophysical objects on various scales, such as stars in galaxies (in particular rotation curves) as well as galaxies and ionized gas clouds within galaxy clusters, and by gravitational lensing (weak and strong).

${ }^{66}$ The fact that most of the matter started to cluster before the decoupling of photons implies that it had decoupled considerable earlier, hence is not composed of (electrically charged) SM particles.
} 
of DM particles ("top down"). This is in contradiction to the observed LSS, which rules out the only potential DM candidate within the SM, the neutrinos $\nu_{L} 67$ In WDM scenarios, structure formation on scales larger than the particles' average free streaming length $\lambda_{D M}$ is similar to CDM, but differences on smaller scales are expected to be visible in various observables, such as the matter power spectrum, halo density profile, halo mass function, subhalo density profile and subhalo mass function. Qualitatively, structures on scales smaller than $\lambda_{D M}$ are suppressed due to the free streaming.

Sterile neutrinos are collisionless and can be very long lived, hence they are an obvious DM candidate. This scenario has been studied by a large number of authors, see e.g. 72, 74, 203, 204, 312, 358, 370, 493 514, 514 535]. Formally, sterile neutrinos are WDM candidates, though this classification is slightly ambiguous because momentum distribution may be very different from Fermi-Dirac. Their properties are constrained by the requirements outlined below. In what follows, we require that all DM is composed of sterile neutrinos. If they make up only a fraction of $\Omega_{D M}$, then the bounds weaken considerably. For instance, sterile neutrinos with eV masses could give a subdominant HDM contribution to $\Omega_{D M}$ if there are other CDM particles that ensure consistency with structure formation.

Stability $-N_{I}$ particles are unstable and decay via the $\theta$-suppressed weak interaction. If they are DM, their lifetime must be longer than the age of the universe.

X-ray bounds - The radiative decay $N_{I} \rightarrow \gamma v_{\alpha}$ via processes as shown in figure 13 predicts the emission of photons with energy $M_{I} / 2$ from DM dense regions [498, 532]68. The non-observation of such signal in the data of different X-ray observatories (such as XMM, Chandra and Suzaku) [203, 495, 497 502, 508, 509, 513, 523 529, 537] imposes upper bounds in the mass-mixing plane; these are displayed in figure 12, In figure 14 X-ray bounds are combined with other constraints. For decaying DM, the signal strength scales only linear with the DM column density along the line of sight, hence one can expect a signal from wider range of astrophysical objects than for annihilating DM. This makes future searches promising despite the fact that there are various astrophysical sources in the keV range. The perspectives for future searches have e.g. been outlined in [27, 516, 532 534]. Finally, it is worth to emphasize that this scenario is falsifiable: if $\mathrm{DM}$ is made of $\mathrm{RH}$ neutrinos that are produced thermally in the early universe through their mixing with active neutrinos, their mass and mixing are constrained in all directions in figure 14 and this scenario can be found or falsified on human time scales.

Phase space analysis - As a fermionic DM candidate, the mass of RH neutrinos is constrained by the Tremaine-Gunn bound [538] on the phase space density in the Milky way's dwarf spheroidal galaxies [512, 514]. This yields a lower bound of $M_{N}>1 \mathrm{keV}$. In [530] it has been shown that phase space arguments on the galactic scale indeed favour a keV DM particle. The same authors have argued in [531] that, due to the high phase space density, a quantum mechanical treatment is necessary on these scales, which may solve the "cusp issue".

\footnotetext{
${ }^{67}$ The active neutrino background can be viewed as a small HDM contribution to the matter density in the universe.

${ }^{68}$ Here we assume that $\nu_{R}$ have (at least) the Yukawa interactions in (1). If such interactions are suppressed, the RH neutrinos that constitute DM can be much heavier and may even be related to the observed excess [10] of $\gamma$-ray emission near the galactic center [536].
} 


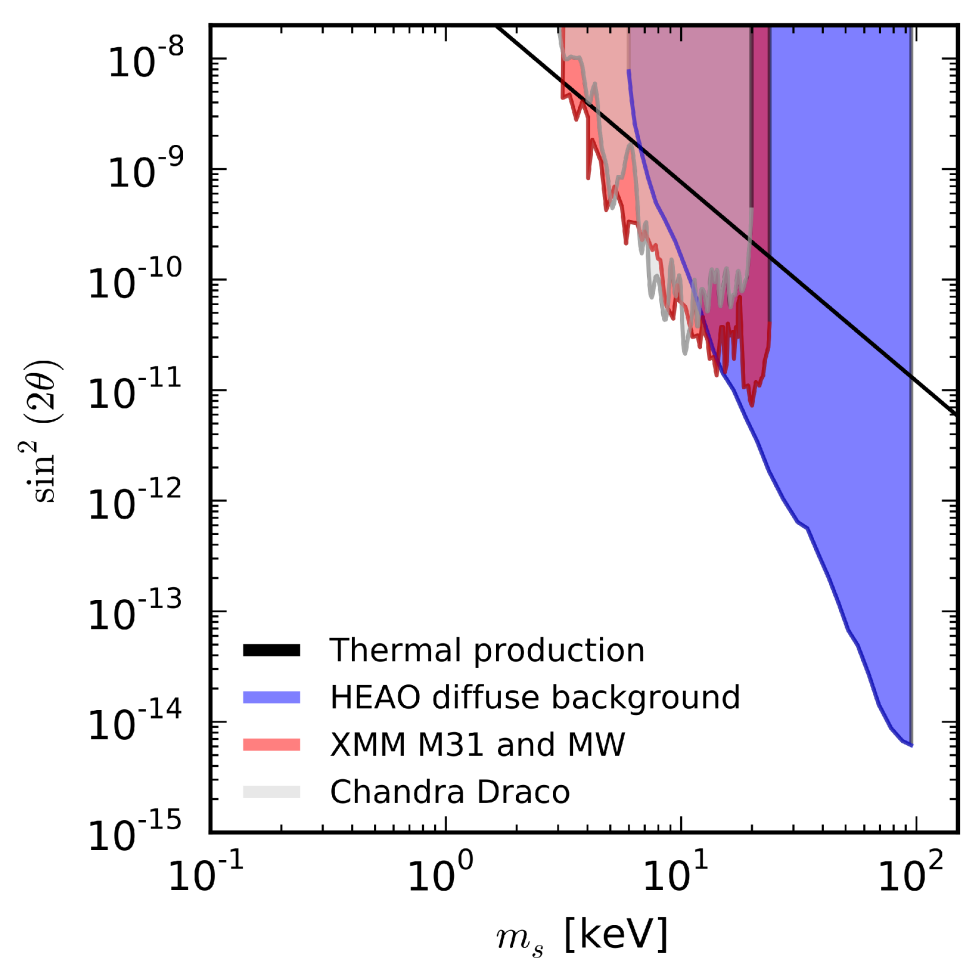

Figure 12: Different constraints on sterile neutrino DM mass and mixing from X-ray observations. The constraints are take from [509] (red), rescaled by a factor of two due to mass estimate uncertainties as recommended in [203], [527] (grey) and [497] (purple). Some analyses have claimed stronger constraints, but were later found to be too optimistic. In [498, 501] it was found that [495] underestimated the flux by two orders of magnitude. According to [509] the mass was overestimated in [500] leading to too restrictive constraints. The constraints in [526] might be too restrictive due to the choice of source profile [508]. The spectral resolution seems to be overestimated in [529], cf. Chandra Proposers Guide; this seems to be the main reason for the stronger bounds used in [136]. Thanks to Signe Riemer-Sørensen for the plot and comments.

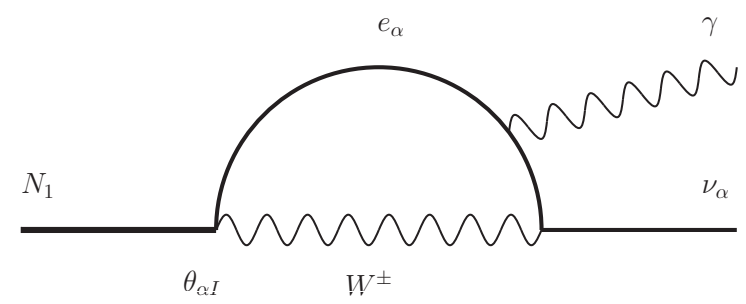

Figure 13: Example for a contribution to the radiative decay of a DM sterile neutrino $N_{1} \rightarrow \gamma v_{\alpha}$. The coupling of $N_{1}$ to the $W$-boson is suppressed by the mixing $\theta_{\alpha 1}$ as indicated.

Production in the early universe It is not known when and how DM was produced in the early universe. If it is composed of sterile neutrinos, then there are several possible production mechanisms.

- Thermal production via mixing (non-resonant) - If $\nu_{R}$ have nonzero Yukawa couplings $F$ (i.e. $m_{D} \neq 0$ ), then they are produced thermally from the primordial plasma via their 


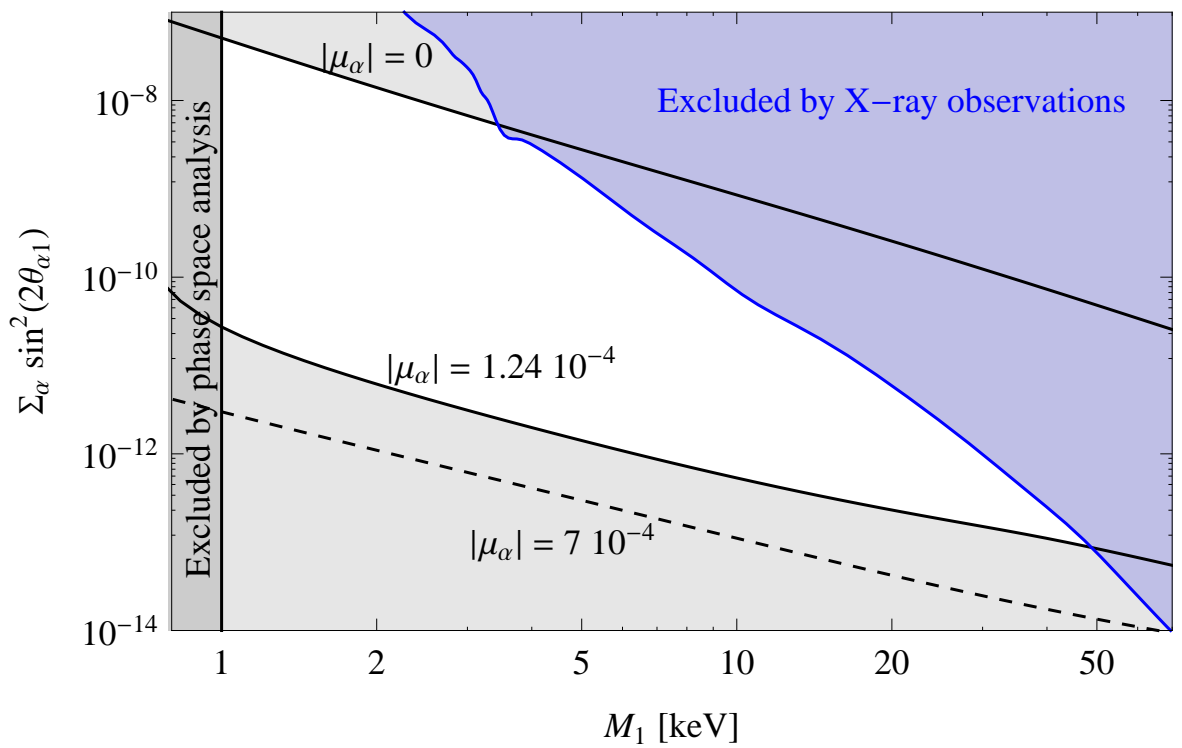

Figure 14: Different constraints on sterile neutrino DM mass and mixing, assuming $\nu_{R}$ have no interactions in addition to the Yukawa couplings in (1). The blue region is excluded by X-ray observations, the dark grey region $M<1 \mathrm{keV}$ by the Tremaine-Gunn bound [512, [514, 538]. The solid black lines are "production curves" for thermal production. For all points on the upper black line the observed $\Omega_{D M}$ is produced in the absence of lepton asymmetries (for $Y_{\alpha}=0$, non-resonant production) [253]. Points on the lower solid black line yield the correct $\Omega_{D M}$ for $\left|Y_{\alpha}\right|=1.24 \cdot 10^{-4}$ at $T=100 \mathrm{MeV}$, the dashed line for $\left|Y_{\alpha}\right|=7 \cdot 10^{-4}$. For these values the resonant production mechanism contributes. The region between these lines is accessible for intermediate values of $Y_{\alpha}$. We do not display bounds derived from structure formation because they depend on $Y_{\alpha}$ in a complicated way and there are considerable uncertainties. Plot taken from [250]. 
mixing with the SM neutrinos $\nu_{L}$ [539]. If this production was efficient enough to bring them into thermal equilibrium, their density would be bigger than $\Omega_{D M}$ and "overclose" the universe unless they are diluted by entropy production at some later stage [540 542]. This puts an upper bound on the mixing angle, given by the upper production curve in figure 14, The production can yield the correct $\Omega_{D M}$ if $\theta$ is small enough that the DM sterile neutrino never reach thermal equilibrium. In that case they still have a momentum distribution that is proportional to a Fermi-Dirac spectrum [494, 539, 543, 544], which makes them a WDM candidate that is at least disfavoured by structure formation arguments (see below).

- Resonant thermal production - The properties of (quasi)particles in the primordial plasma are modified by the interactions with the medium [380, 545, 546]. In the presence of a lepton asymmetry, the Mikheev-Smirnov-Wolfenstein effect [547, 548] can lead to a level crossing between active and sterile neutrino dispersion relations [253, 549]. This results in a resonant enhancement of the sterile neutrino production rate. The resulting spectrum is nonthermal, with higher occupation numbers for low momentum modes [253]. Production curves for two different values of the asymmetry are also given in figure 14. Note that these have to be taken with some care because in the calculation of the production efficiency [253] it was assumed that $Y_{e}=Y_{\nu}=Y_{\tau}$. In reality the asymmetries in different flavours may be different and can even have opposite sign (see e.g. [251] for a particular model).

- Thermal production beyond the SM - The fields $\nu_{R}$ are singlet under SM the gauge group and only interact with SM particles via their Yukawa couplings $F$. However, if there exists and extended "hidden sector" [550] or extended Higgs sector, they may have additional interactions with these. They may also be charged under some extended gauge group that is broken at high energies, as e.g. in the left-right-symmetric model [522]. Such additional interactions would contribute to the thermal production [541] in the early universe. Since they usually increase the production rate, the universe may overclose unless the DM abundance is diluted by additional entropy production at later times [551].

- Nonthermal production - RH neutrinos can also be produced nonthermally, e.g. due to a coupling to an inflaton [552 554], the SM Higgs [555], other scalars [166, 556] or modified gravity [557].

All these scenarios are constrained by the requirements to produce the correct $\Omega_{D M}$ and a momentum distribution that is consistent with the observed LSS.

Structure formation - The masses dictated by the above bounds suggest that, if RH neutrinos mix with LH neutrinos, then they formally are WDM, but their momentum distribution can be non-thermal. Structure formation in WDM scenarios on scales above the free streaming length is similar to CDM. On smaller scales the formation of structures is affected in different ways, the study of which is in general complicated due to the nonlinear nature of the clustering process. Most importantly, one expects a cut in the matter power spectrum at scales below the free streaming length [558]. In addition, the suppression of small scale structures should be visible in the halo mass function (which counts the number of haloes per unit volume per unit mass at given redshift) [559] and affect the gravitational collapse leading to the formation of the first stars [560, 561]. Unfortunately, these arguments do not directly constrain $M_{N}$, but the free streaming length. Furthermore, structure formation is a highly nonlinear problem in the regime 
$\delta \rho_{k} / \rho>1$ that can only be studied quantitatively with expensive many body simulations. With few exceptions [312], these simulations assume that the DM distribution is proportional to a Fermi-Dirac distribution [494]. If this is the case, then the comoving free streaming length $\lambda_{D M}$ is simply related to the mass by [534, 558]

$$
\lambda_{D M} \sim 1 \mathrm{Mpc}\left(\frac{\mathrm{keV}}{M}\right) \frac{\left\langle p_{N}\right\rangle}{\left\langle p_{v}\right\rangle},
$$

where $p_{N}$ and $p_{v}$ are the spatial momenta of sterile and active neutrinos and $M$ is the sterile neutrino mass. Such spectra are produced by the non-resonant thermal production from the primordial plasma. In that case $\mathrm{Ly} \alpha$ forest observations strongly constrain the viability of WDM [312, 504 506, 510, 519, 520, 562 567]; for sterile neutrinos they impose a lower bound of $M_{N}>8$ $\mathrm{keV}$ 510]. Then it may be very difficult to distinguish RH neutrino WDM from CDM observationally [510, 568], see also [569]. If, on the other hand, RH neutrino DM is produced by some other mechanism and has a nonthermal spectrum, the relation (40) between mass and free streaming length does not apply, and simulations of structure formation based on thermal WDM do not allow to draw any general conclusions. In case of the resonant thermal production mechanism, the resulting spectrum has been calculated for $Y_{e}=Y_{\mu}=Y_{\tau}$ [203, 253]. It can be viewed as a superposition of a WDM component and a nonthermal cold component [510, 511], where the Ly $\alpha$ forest bounds allow the warm component to be much "warmer" than in conventional WDM scenarios. First simulations [312] indicate that this scenario may perform better than CDM in predicting the abundance of small scale structures (such as satellite/dwarf galaxies), but the question is not settled at this stage. On one hand, only little is known about how much structure actually exists at small (sub-galactic) scales. While lensing flux and stellar streams suggest the existence of subhalos [492], there is no direct observation of such structures and their existence is disfavoured by some stability considerations [570]. On the other hand there are no systematic studies of structure formation in the nonlinear regime that include the nonthermal component. Arguments against sterile neutrino DM are essentially based on extrapolations of results from simulations that assume purely thermal spectra.

\section{$7 \quad$ A theory of almost everything}

Right handed neutrinos $\nu_{R}$, described by the Lagrangian (1), provide plausible explanations for the phenomena (I), (II), (III), (i) and (ii) named in the introduction. In this section we address the question how many of these phenomena can be explained simultaneously by $\mathrm{RH}$ neutrinos alone.

Minimal case $n=3$ - The Lagrangian (1) with $n=3 \mathrm{RH}$ neutrinos and $M_{M}$ at or below the electroweak scale [571, 572] is an extension of the SM motivated by the principle of minimality (or Ockham's razor); it makes only minimal modifications to the known principles and particles in nature. There is no modification to the SM gauge group, the number of fermion families is unchanged, there is no modification to the bosonic field content of the SM or the mechanism of electroweak symmetry breaking, and no new scale above the electroweak scale is introduced 69 . In [250] it has been shown that this model can indeed simultaneously explain (I)-(III); that is, all known evidence for particle physics beyond the SM. This minimal scenario is known as Neutrino

\footnotetext{
${ }^{69}$ This implies that the hierarchy problem of the SM can be avoided [573 575$]$.
} 
Minimal Standard Model ( $\nu \mathrm{MSM})$. Various aspects of this model have been studied in the past by different authors 119, 132, 133, 147, 148, 151, 203, 250, 253, 266, 422, 424, 468, 497, 498, 524, 540, 544, 552, 553, 555, 571, 572, 574 588], see [203, 251, 589] for a detailed introduction and review.

In the $\nu \mathrm{MSM}$, two sterile neutrinos $\left(N_{2,3}\right)$ have degenerate masses between roughly a $\mathrm{GeV}$ and the electroweak scale; the third one $\left(N_{1}\right)$ has a mass in the keV range and acts as DM candidate. $N_{2,3}$ generate active neutrino masses via the seesaw mechanism; at the same time, the CP-violating oscillations during their thermal production at temperatures $T>T_{E W}$ produce the baryon asymmetry of the universe via the mechanism outlined in section 5.2. The lepton asymmetries generated during $N_{2,3}$ production are washed out after sphaleron freezeout at $T \sim$ $T_{E W}$. The freezeout $\left(T \sim M_{2,3}\right)$ and decay $\left(T<M_{2,3}\right)$ of the $N_{2,3}$ particles produce new, late time lepton asymmetries, which can be orders of magnitude bigger than the baryon asymmetry. The late time asymmetries are capable of enhancing the rate of $N_{1}$ production sufficiently to explain the observed $\Omega_{D M}$ for $\left|Y_{\alpha}\right| \gtrsim 10^{-5}$, while being in agreement with all known constraints on DM properties.

The minimal extension of the particle content in the $\nu$ MSM comes at the price of a "fine tuning" in the mass splitting of two RH neutrinos: $M_{2}$ and $M_{3}$ have to be equal to a level of precision $\left|M_{2}-M_{3}\right| /\left(M_{2}+M_{3}\right) \sim 10^{-13}[251]$. This degeneracy may be related to new symmetries [119, 582], but cannot be explained in the framework of the $\nu$ MSM itself. The tuning mainly arises from the requirement to produce a late time lepton asymmetry $\left|Y_{\alpha}\right| \gtrsim 10^{-5}$; this is necessary to sufficiently enhance the $N_{1}$ production to explain the observed $\Omega_{D M}$. If some of the asymmetries generated before sphaleron freezeout can be preserved from washout [590] or $N_{1}$ is produced by some other mechanism (e.g. during reheating), then the required degree of degeneracy reduces to $\sim 10^{-3}$. This weaker degeneracy is necessary for baryogenesis, i.e. to explain the observed $\Omega_{B}$ from CP-violating oscillations of two sterile neutrinos $N_{2,3}$. The third sterile neutrino $N_{1}$ cannot contribute significantly to leptogenesis if it is DM because in this case its couplings $F_{\alpha 1}$ are constrained to be tiny by X-ray bounds on its decay width, cf. section 6 . For the same reason it also cannot make a significant contribution to neutrino masses via the seesaw mechanism (14); this implies that the lightest active neutrino is effectively massless in the $\nu$ MSM. The smallness of the Yukawa couplings also implies that the lepton flavour violation they induce is too small to be observed in muon-to-electron conversion experiments in foreseeable time [589]. Constraints from to $0 \nu \beta \beta$-decays currently also do not constrain the allowed region, but will be relevant when $m_{e e}$ is probed at the level of $10^{-2} \mathrm{eV}[134] 70$

The particles $N_{2}$ and $N_{3}$ can be searched for in collider experiments, cf. section 3.4. They also lead to $L$-violation that may be seen in $0 \nu \beta \beta$-decay searches [133, 134]. Known bounds on their mass and mixing are summarized in figure 15. The DM candidate $N_{1}$ is too weakly coupled to be studied at colliders, but can be found by indirect DM searches for the X-ray emission line from the decay $N_{1} \rightarrow \gamma v$, cf. figure 13 . Its properties are also constrained by phase space and structure formation considerations. Figure 14 summarizes different bounds on $N_{1}$ mass and mixing.

More than three sterile neutrinos - The required amount of "fine tuning" can be considerably reduced if there are more than three sterile neutrinos. For instance, if three sterile neutrinos participate in baryogenesis, then there is no need for degenerate masses even if they are as light as a few $\mathrm{GeV}$ [422]. If one at the same time requires one sterile neutrino with $\mathrm{keV}$

\footnotetext{
${ }^{70}$ The contributions from $N_{I}$ exchange in the $\nu$ MSM are negligible [132] or negative [133].
} 
mass to compose DM, then that means there must be at least $n=4$ of them altogether. If one, in addition to the evidence (I)-(III), also wants to address the hints (i) and (ii) in this framework, then there must be least one more sterile neutrino with an $\mathrm{eV}$ mass. This light sterile neutrino, responsible for the oscillation anomalies, would leave the thermal history of the universe before BBN essentially unaffected due to the smallness of its couplings enforced by the seesaw relation (14). At the same time it can be responsible for the $N_{\text {eff }}>3$ inferred from BBN and the CMB, see section 4. The late time lepton asymmetries generated by the freezeout and decay of its $M_{I} \gtrsim 1 \mathrm{GeV}$ siblings could help to suppress the efficiency of its thermal production and ease the tension between oscillation anomaly and dark radiation signals, see section 4.2. Thus, $n \geq 4 \mathrm{RH}$ neutrinos described by the Lagrangian (1) alone can be capable of explaining the points (I)-(III) as well as (i) and (ii) simultaneously without "new physics" above the electroweak scale; that is all confirmed fundamental physics phenomena that cannot be explained in the SM except those that are likely to involve accelerated cosmic expansion. Hence, this framework at the current stage provides a phenomenologically complete description of particle physics.

\section{Conclusions}

Right handed neutrinos provide plausible explanations for neutrino oscillations, the observed dark matter and the baryon asymmetry of the universe, which cannot be understood within the SM. They may also be responsible for the anomalies reported by some short baseline neutrino experiments and hints for dark radiation in cosmological data. Right handed neutrinos can be embedded naturally into bigger frameworks, such as left-right symmetric theories, grand unified theories, supersymmetric extensions of the SM and various models of neutrino mass generation. The different phenomena point towards different right handed neutrino mass ranges, which may of course coexist in nature. Several of these can be probed by direct and indirect laboratory searches, astrophysical observations and cosmological data in the near future. We summarize well-motivated mass ranges and their phenomenological relevance in the overview table appendix A.

\section{Acknowledgements}

I would like to thank Signe Riemer-Sørensen and Yvonne Wong for valuable discussions on astrophysical and cosmological bounds as well as Fedor Bezrukov for his explanations on neutrinoless double $\beta$-decay. Thanks also to Oleg Ruchayskiy and Viviana Niro for their comments on the interplay between X-ray bounds and neutrinoless double $\beta$-decay. I am very grateful to Björn Garbrecht, Pasquale Di Bari, Fedor Bezrukov, Alexander Merle, Signe Riemer-Sørensen and Yvonne Wong for their comments on individual sections and to Mikhail Shaposhnikov for proof-reading of the manuscript. Finally, I would like to thank Nancy Lu for her patience on the numerous evenings I spent writing this review during the past few months. This work was supported by the Gottfried Wilhelm Leibniz program of the Deutsche Forschungsgemeinschaft. 

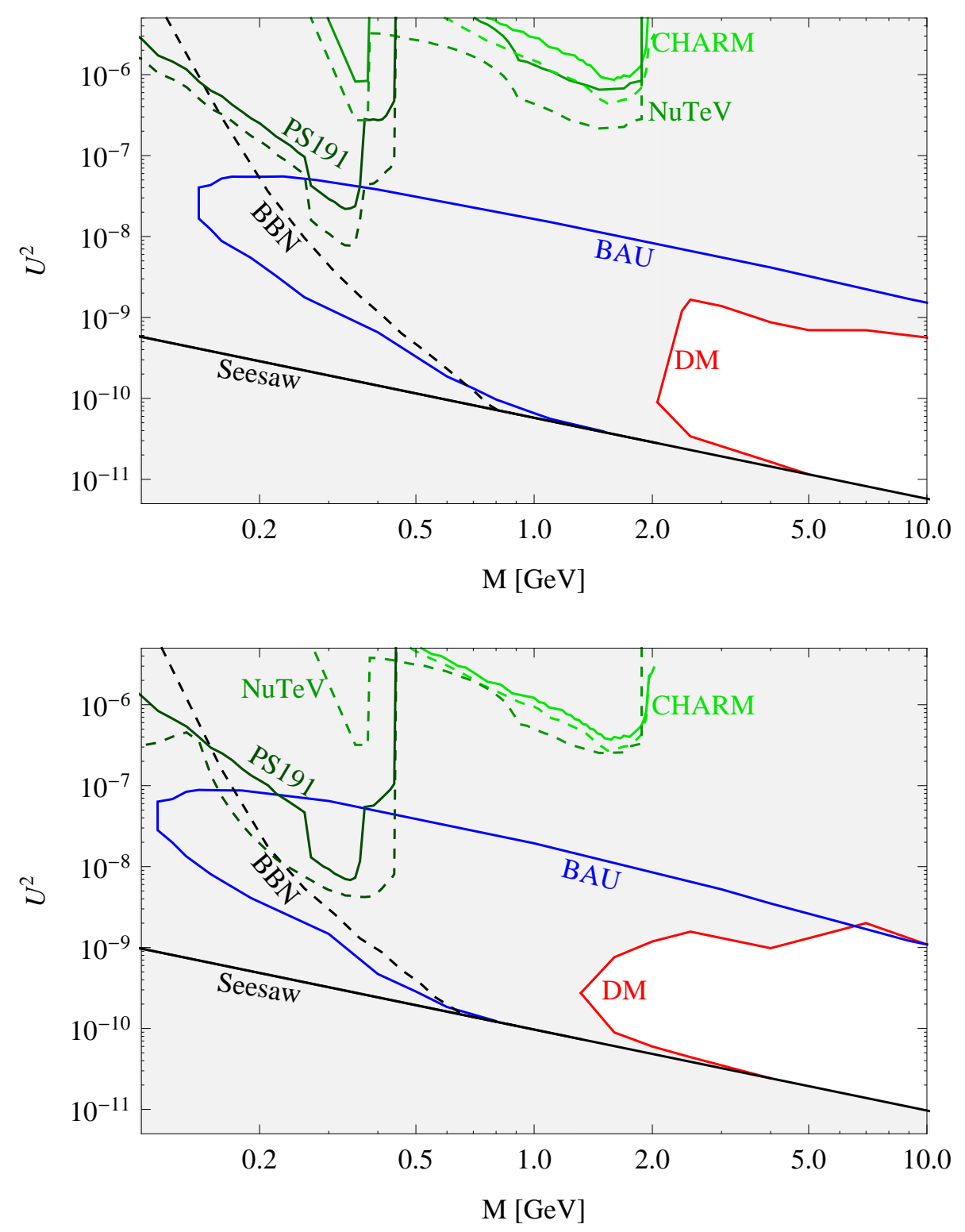

Figure 15: Constraints on the mean value $M$ of the degenerate $N_{2,3}$ masses $M_{2,3} \simeq M$ and mixing $U^{2}=\operatorname{tr}\left(\theta^{\dagger} \theta\right)$ in the $\nu M S M$; upper panel - normal hierarchy, lower panel - inverted hierarchy. In the region between the solid blue "BAU" lines, the observed $\Omega_{B}$ can be generated. The observed $\Omega_{D M}$ can be explained in terms of sterile neutrinos inside the solid red "DM" line (there the lepton asymmetry at $T=100 \mathrm{MeV}$ can be large enough to resonantly enhance the $N_{1}$ production). The $C P$-violating phases were chosen to maximize the asymmetry at $T=100 \mathrm{MeV}$. The regions below the solid black "seesaw" line and dashed black "BBN" line are excluded by neutrino oscillation experiments and BBN, respectively. The areas above the green lines of different shade are excluded by direct search experiments, as indicated in the plot. The solid lines are exclusion plots for all choices of $\nu M S M$ parameters, for the dashed lines the phases were chosen to maximize the late time asymmetry, consistent with the red line. We do not show constraints from to $0 \nu \beta \beta$-decays, which currently do not constrain the allowed region, but will be relevant when $m_{e e}$ is probed at the level of $10^{-2} \mathrm{eV}$ [134]. Plot taken from [250]. 


\section{A Overview Table: Majorana mass scales and observables}

\begin{tabular}{|c|c|c|c|c|c|c|c|}
\hline$M_{M}$ & Motivation & v-oscillations & laboratory searches & CMB & $\mathrm{BBN}$ & $\mathrm{DM}$ & Leptogenesis \\
\hline$\lesssim \mathrm{eV}$ & $\begin{array}{c}\text {-oscillations anomalies, } \\
\text { dark radiation }\end{array}$ & $\begin{array}{l}\text { massses by seesaw, }{ }^{a} \\
\text { explain anomalies }^{b}\end{array}$ & $\begin{array}{c}\text { oscillation anomalies, } \\
\beta \text {-decays }\end{array}$ & explain $N_{\text {eff }}>3^{b}$ & may explain $N_{\text {eff }}>3^{b}$ & no & no \\
\hline $\mathrm{keV}$ & $\mathrm{DM}$ & no if $\mathrm{DM}^{c}$ & $\begin{array}{l}\text { direct searches? } \\
\text { nuclear decays? }\end{array}$ & $\begin{array}{c}\text { act as DM, } \\
\text { no effect on } N_{\text {eff }}\end{array}$ & $\begin{array}{l}\text { effect on } N_{\text {eff }} \\
\text { too small if DM }\end{array}$ & good candidate & no \\
\hline $\mathrm{MeV}$ & testability, why not? & masses by seesaw & $\begin{array}{c}\text { intensity frontier, } \\
0 \nu \beta \beta \\
\end{array}$ & unaffected & $\begin{array}{c}\text { constrains } \\
M_{I} \gtrsim 200 \mathrm{MeV} \\
\end{array}$ & no ${ }^{e}$ & $\begin{array}{c}\text { possible } \\
\text { (fine tuning) }\end{array}$ \\
\hline $\mathrm{GeV}$ & $\begin{array}{l}\text { testability, } \\
\text { minimality }\end{array}$ & masses by seesaw & $\begin{array}{c}\text { intensity frontier, } \\
\mathrm{EW} \text { precision data, } \\
0 \vee \beta \beta\end{array}$ & unaffected & unaffected & no ${ }^{e}$ & possible \\
\hline $\mathrm{TeV}$ & $\begin{array}{c}\text { minimality, } \\
\text { testability }\end{array}$ & masses by seesaw & LHC & unaffected & unaffected & no ${ }^{e}$ & possible \\
\hline$\gtrsim 10^{9} \mathrm{GeV}$ & $\begin{array}{c}\text { grand unification, } \\
\text { "naturally" small } v \text {-masses }\end{array}$ & masses by seesaw & too heavy to be found & unaffected & unaffected & no $^{e}$ & works naturally \\
\hline
\end{tabular}

Colour code: green $=$ can affect, red $=$ does not affect

${ }^{a}$ This assumes that the observed $\Delta m_{\text {atm }}$ and $\Delta m_{\text {sol }}$ are generated by $\nu_{R}$ other than those responsible for the anomalies, i.e. $n>2$.

${ }^{b}$ Sterile neutrinos with masses and mixings suggested by the oscillation anomalies would be in thermal equilibrium in the early universe, hence increase $N_{\text {eff }}$ by one unit $\stackrel{\infty}{\infty} \quad$ per species. In $\Lambda \mathrm{CDM}$ cosmology, this is in conflict with recent CMB data and HDM constraints. If some mechanism prevents the sterile neutrinos from thermalizing or there are deviations from the standard $\Lambda \mathrm{CDM}$ model, this conflict can be avoided.

${ }^{c} \nu_{R}$ with keV-masses can generate neutrino masses via the seesaw mechanism, but then they are too short lived to be DM. If they are DM, then their Yukawa coupling is too small to contribute to the seesaw - they can either be DM or contribute to the seesaw mechanism.

${ }^{d}$ It is disputed whether the signal can be distinguished from the active neutrino background; for the case that keV sterile neutrinos compose all DM the author considers it very unlikely that such searches are successful because of the astrophysical constraints on the mixing angle.

e This applies to sterile neutrinos thermally produced via their mixing. Sterile neutrinos with $M_{I} \gg \operatorname{keV}$ can be DM if $F \simeq 0$ ensures their stability and the production in the early universe is due to an unknown interaction. 


\section{B Dirac and Majorana masses}

This appendix summarizes some basic aspects of Majorana fermions, see [24, 25] for details. In the SM, matter is composed of fermions. In relativistic quantum field theory these can be described by two component (Weyl) spinors $\psi_{L}$ and $\psi_{R}$, which transform under the (irreducible) left or right handed representations of the Poncairé group, respectively. For historical [591] and computational reasons fermions are, however, often represented by four component spinors [592]. In this review we adopt this common "overnotation"; using the Weyl representation of $\gamma^{\mu}$ matrices, we define four component spinors $\Psi_{L}=\left(\psi_{L}, 0\right)^{T}$ and $\Psi_{R}=\left(0, \psi_{R}\right)^{T}$. Consider sets of left and right handed spinors $\Psi_{L, i}$ and $\Psi_{R, j}$, where the indices $i$ and ${ }_{j}$ run from 1 to $n$ and $m$, respectively, and label individual fields ("flavours"). The most general free Lagrangian reads

$$
\frac{i}{2}\left(\overline{\Psi_{L}} \partial \Psi_{L}+\overline{\Psi_{R}} \partial \Psi_{R}\right)-\overline{\Psi_{L}} m_{D} \Psi_{R}-\frac{1}{2}\left(\overline{\Psi_{L}} m_{M} \Psi_{L}^{c}+\overline{\Psi_{R}} M_{M} \Psi_{R}^{c}\right)+\text { h.c. }
$$

where we have suppressed flavour indices $\left(\Psi_{R}, \Psi_{L}\right.$ are now flavour vectors with components $\Psi_{R, i}$ and $\left.\Psi_{L, j}\right)$. The matrices $m_{D}, m_{M}$ and $M_{M}$ can be combined into a $(n+m) \times(n+m)$ matrix $\mathfrak{M}$, the physical mass squares are the eigenvalues of $\mathfrak{M M}^{\dagger}$. The mass term reads

$$
\frac{1}{2}\left(\overline{\Psi_{L}} \overline{\Psi_{R}^{c}}\right) \mathfrak{M}\left(\begin{array}{c}
\Psi_{L}^{c} \\
\Psi_{R}
\end{array}\right)+\text { h.c. } \equiv \frac{1}{2}\left(\overline{\Psi_{L}} \overline{\Psi_{R}^{c}}\right)\left(\begin{array}{cc}
m_{M} & m_{D} \\
m_{D}^{T} & M_{M}^{\dagger}
\end{array}\right)\left(\begin{array}{c}
\Psi_{L}^{c} \\
\Psi_{R}
\end{array}\right)+\text { h.c. }
$$

All fermions in the SM are charged under some gauge group, thus any intrinsic mass terms are forbidden by gauge invariance. Terms as $m_{D}$ are generated by the Higgs mechanism. For $M_{M}=0, m_{M}=0$ one can combine pairs of left and right handed fields in (41) into Dirac spinors $\Psi_{i} \equiv \Psi_{R, i}+\Psi_{L, i}$ and write $\mathcal{L}=\bar{\Psi}_{i}\left(i \not \partial-m_{D}\right) \Psi_{i}$.71 This is the reason why one can describe charged leptons and quarks by Dirac spinors though the basic building blocks of matter are Weyl fermions: the conserved charge related to the unbroken $U(1)$ subgroup in the electroweak theory leads to mass matrix that allows to combine all Weyl fields into Dirac spinors. If, on the other hand, $m_{M}, M_{M} \neq 0$, then one can form $n+m$ Majorana spinors [593]. In the simplest case, when $m_{D}=0$ and $m_{M}$ and $M_{M}$ are diagonal, one can define these as $\chi_{i}=\Psi_{L, i}+\Psi_{L, i}^{c}$ and $\psi_{j}=\Psi_{R, j}+\Psi_{R, j}^{c}$. Formally $\chi_{i}$ and $\psi_{i}$ are four component objects, and the Lagrangian can be written in analogy to the Dirac equation, $\frac{1}{2}\left(\bar{\chi}\left(i \not \partial-m_{M}\right) \chi+\bar{\psi}\left(i \not \partial-M_{M}\right) \psi\right)$, but they obey the additional Majorana conditions $\chi_{i}=\chi_{i}^{c}, \psi_{i}=\psi_{i}^{c}$ and have only two independent components. Due to this property the neutral fermions can annihilate with themselves (are their own antiparticles).

Because of these considerations $m_{D}$ is usually referred to as Dirac mass term, while $m_{M}$ and $M_{M}$ are called Majorana mass terms. However, apart from the two special cases discussed above, they have not much to do with the appearance of Dirac or Majorana particles. Even for $m_{D}=0$ the particle spectrum may contain Dirac spinors. Consider, for instance, a set of three fields $\Psi_{L, 1}$, $\Psi_{R, 1}$ and $\Psi_{R, 2}$ with $m_{D}, m_{M}=0$ and $M_{M}=M \mathbb{1}_{2 \times 2}$. Then one can combine $\Psi_{R, 1}$ and $\Psi_{R, 2}$ into a Dirac spinor $\Psi=\left(i \psi_{1}+\psi_{2}\right) / \sqrt{2}$. On the other hand, one can split any Dirac spinor into two Majorana spinors in this way.

Thus, fermions can generically described by two component Weyl spinors. If one, for computational convenience, prefers to use four component spinors, one can use the chiral spinors $\Psi_{R, L}$. Only in special cases one can combine these into Dirac spinors. This is possible whenever the full

\footnotetext{
${ }^{71}$ For $m \neq n$ or if not all eigenvalues of $M_{M}$ are vanishing this is obviously not possible for all $\Psi_{L, i}$ and $\Psi_{R, j}$.
} 
mass matrix $\mathfrak{M}$ has degenerate eigenvalues (with opposite sign), and not necessarily related to the presence of "Dirac" or "Majorana mass terms".

\section{References}

[1] M. Drewes, The Phenomenology of Right Handed Neutrinos, International Journal of Modern Physics E, Vol. 22 (2013) 1330019.

[2] Particle Data Group Collaboration, J. Beringer et al., Review of Particle Physics (RPP), Phys.Rev. D86 (2012) 010001.

[3] F. Jegerlehner and A. Nyffeler, The Muon g-2, Phys.Rept. 477 (2009) 1-110, arXiv:0902.3360].

[4] DAMA Collaboration, LIBRA Collaboration Collaboration, R. Bernabei et al., New results from DAMA/LIBRA, Eur.Phys.J. C67 (2010) 39-49, [arXiv:1002.1028].

[5] PAMELA Collaboration Collaboration, O. Adriani et al., An anomalous positron abundance in cosmic rays with energies 1.5-100 GeV, Nature 458 (2009) 607-609, [arXiv:0810.4995].

[6] AMS Collaboration Collaboration, M. Aguilar et al., First Result from the Alpha Magnetic Spectrometer on the International Space Station: Precision Measurement of the Positron Fraction in Primary Cosmic Rays of 0.5350 GeV, Phys.Rev.Lett. 110 (2013), no. 14141102.

[7] CDF Collaboration Collaboration, T. Aaltonen et al., Evidence for a Mass Dependent Forward-Backward Asymmetry in Top Quark Pair Production, Phys.Rev. D83 (2011) 112003, arXiv:1101.0034.

[8] H. Klapdor-Kleingrothaus, A. Dietz, H. Harney, and I. Krivosheina, Evidence for neutrinoless double beta decay, Mod.Phys.Lett. A16 (2001) 2409-2420, hep-ph/0201231.

[9] B. D. Fields, The primordial lithium problem, Ann.Rev.Nucl.Part.Sci. 61 (2011) 47-68, [arXiv:1203.3551].

[10] C. Weniger, A Tentative Gamma-Ray Line from Dark Matter Annihilation at the Fermi Large Area Telescope, JCAP 1208 (2012) 007, [arXiv:1204.2797].

[11] J. A. King, J. K. Webb, M. T. Murphy, V. V. Flambaum, R. F. Carswell, et al., Spatial variation in the fine-structure constant - new results from VLT/UVES, MNRAS $\mathbf{4 2 2}$ (2012) 3370, arXiv:1202.4758.

[12] H. Murayama, H. Suzuki, T. Yanagida, and J. Yokoyama, Chaotic inflation and baryogenesis by right-handed sneutrinos, Phys.Rev.Lett. 70 (1993) 1912-1915.

[13] A. Mazumdar and S. Morisi, Split neutrinos, two Majorana and one Dirac, and implications for leptogenesis, dark matter, and inflation, Phys.Rev. D86 (2012) 045031, [arXiv:1201.6189]. 
[14] M. Shaposhnikov and C. Wetterich, Asymptotic safety of gravity and the Higgs boson mass, Phys. Lett. B683 (2010) 196-200, arXiv:0912.0208.

[15] F. Bezrukov, M. Y. Kalmykov, B. A. Kniehl, and M. Shaposhnikov, Higgs Boson Mass and New Physics, JHEP 1210 (2012) 140, arXiv:1205.2893.

[16] G. Degrassi, S. Di Vita, J. Elias-Miro, J. R. Espinosa, G. F. Giudice, et al., Higgs mass and vacuum stability in the Standard Model at NNLO, JHEP 1208 (2012) 098, arXiv:1205.6497.

[17] S. Alekhin, A. Djouadi, and S. Moch, The top quark and Higgs boson masses and the stability of the electroweak vacuum, Phys.Lett. B716 (2012) 214-219, [arXiv:1207.0980].

[18] J. Elias-Miro, J. R. Espinosa, G. F. Giudice, G. Isidori, A. Riotto, et al., Higgs mass implications on the stability of the electroweak vacuum, Phys.Lett. B709 (2012) 222-228, arXiv:1112.3022].

[19] ALEPH Collaboration, DELPHI Collaboration, L3 Collaboration, OPAL Collaboration, SLD Collaboration, LEP Electroweak Working Group, SLD Electroweak Group, SLD Heavy Flavour Group Collaboration, S. Schael et al., Precision electroweak measurements on the $Z$ resonance, Phys.Rept. 427 (2006) 257-454, hep-ex/0509008.

[20] D. Delepine, C. Lujan-Peschard, and M. Napsuciale, A Fourth Neutrino and its Consequences on CP Asymmetries, arXiv:1303.4687.

[21] M. Shaposhnikov, Neutrino physics within and beyond the three flavour oscillation, J.Phys.Conf.Ser. 408 (2013) 012015.

[22] ATLAS Collaboration Collaboration, G. Aad et al., Observation of a new particle in the search for the Standard Model Higgs boson with the ATLAS detector at the LHC, Phys.Lett.B (2012) arXiv:1207.7214.

[23] CMS Collaboration Collaboration, S. Chatrchyan et al., Observation of a new boson at a mass of $125 \mathrm{GeV}$ with the CMS experiment at the LHC, Phys.Lett.B (2012) arXiv:1207.7235].

[24] R. Mohapatra and P. Pal, Massive neutrinos in physics and astrophysics. Third edition, World Sci.Lect.Notes Phys. 72 (2004) 1-476.

[25] M. Fukugita and T. Yanagida, Physics of neutrinos and applications to astrophysics, Springer (Berlin) (2003) 1-593.

[26] R. Mohapatra, S. Antusch, K. Babu, G. Barenboim, M.-C. Chen, et al., Theory of neutrinos: A White paper, Rept.Prog.Phys. 70 (2007) 1757-1867, hep-ph/0510213.

[27] K. Abazajian, M. Acero, S. Agarwalla, A. Aguilar-Arevalo, C. Albright, et al., Light Sterile Neutrinos: A White Paper, arXiv:1204.5379.

[28] S. Antusch, Models for Neutrino Masses and Mixings, arXiv:1301.5511. 
[29] A. Merle, keV Neutrino Model Building, arXiv:1302.2625.

[30] H. Georgi and S. L. Glashow, Unity of All Elementary Particle Forces, Phys. Rev. Lett. 32 (1974) 438-441.

[31] H. Georgi, The State of the Art-Gauge Theories. (Talk), AIP Conf.Proc. 23 (1975) $575-582$.

[32] H. Fritzsch and P. Minkowski, Unified Interactions of Leptons and Hadrons, Annals Phys. 93 (1975) 193-266.

[33] F. Maltoni, J. Niczyporuk, and S. Willenbrock, Upper bound on the scale of Majorana neutrino mass generation, Phys.Rev.Lett. 86 (2001) 212-215, hep-ph/0006358.

[34] K. R. Dienes, E. Dudas, and T. Gherghetta, Neutrino oscillations without neutrino masses or heavy mass scales: A Higher dimensional seesaw mechanism, Nucl.Phys. B557 (1999) 25, hep-ph/9811428.

[35] N. Arkani-Hamed, S. Dimopoulos, G. Dvali, and J. March-Russell, Neutrino masses from large extra dimensions, Phys.Rev. D65 (2002) 024032, hep-ph/9811448.

[36] Y. Grossman and M. Neubert, Neutrino masses and mixings in nonfactorizable geometry, Phys.Lett. B474 (2000) 361-371, hep-ph/9912408.

[37] N. Arkani-Hamed and Y. Grossman, Light active and sterile neutrinos from compositeness, Phys.Lett. B459 (1999) 179-182, hep-ph/9806223.

[38] A. Strumia and F. Vissani, Neutrino masses and mixings and.., hep-ph/0606054 (2006) hep-ph/0606054.

[39] S. Weinberg, Baryon and Lepton Nonconserving Processes, Phys.Rev.Lett. 43 (1979) 1566-1570.

[40] Z. Maki, M. Nakagawa, and S. Sakata, Remarks on the unified model of elementary particles, Prog.Theor.Phys. 28 (1962) 870-880.

[41] B. Pontecorvo, Neutrino Experiments and the Problem of Conservation of Leptonic Charge, Sov.Phys.JETP 26 (1968) 984-988.

[42] G. Fogli, E. Lisi, A. Marrone, D. Montanino, A. Palazzo, et al., Global analysis of neutrino masses, mixings and phases: entering the era of leptonic CP violation searches, Phys.Rev. D86 (2012) 013012, arXiv:1205.5254].

[43] M. Gonzalez-Garcia, M. Maltoni, J. Salvado, and T. Schwetz, Global fit to three neutrino mixing: critical look at present precision, JHEP 1212 (2012) 123, arXiv:1209.3023.

[44] D. Forero, M. Tortola, and J. Valle, Global status of neutrino oscillation parameters after Neutrino-2012, Phys.Rev. D86 (2012) 073012, [arXiv:1205.4018.

[45] S. Petcov, The Nature of Massive Neutrinos, arXiv:1303.5819. 
[46] V. Antonelli, L. Miramonti, C. Pena-Garay, and A. Serenelli, Solar Neutrinos, Adv.High Energy Phys. 2013 (2013) 351926, [arXiv:1208.1356].

[47] V. Antonelli and L. Miramonti, ADVANCEMENTS IN SOLAR NEUTRINO PHYSICS, Int. J. Mod. Phys. E 22 (2013) 1330009.

[48] Planck Collaboration Collaboration, P. Ade et al., Planck 2013 results. XVI. Cosmological parameters, arXiv:1303.5076.

[49] S. Riemer-Srensen, D. Parkinson, and T. M. Davis, Combining Planck with Large Scale Structure gives strong neutrino mass constraint, arXiv:1306.4153.

[50] C. Froggatt and H. B. Nielsen, Hierarchy of Quark Masses, Cabibbo Angles and CP Violation, Nucl.Phys. B147 (1979) 277.

[51] A. Zee, A Theory of Lepton Number Violation, Neutrino Majorana Mass, and Oscillation, Phys.Lett. B93 (1980) 389.

[52] J. Schechter and J. Valle, Neutrino Masses in SU(2) x U(1) Theories, Phys.Rev. D22 (1980) 2227.

[53] G. Gelmini and M. Roncadelli, Left-Handed Neutrino Mass Scale and Spontaneously Broken Lepton Number, Phys.Lett. B99 (1981) 411.

[54] M. Magg and C. Wetterich, NEUTRINO MASS PROBLEM AND GAUGE HIERARCHY, Phys.Lett. B94 (1980) 61.

[55] T. Cheng and L.-F. Li, Neutrino Masses, Mixings and Oscillations in SU(2) $x$ U(1) Models of Electroweak Interactions, Phys.Rev. D22 (1980) 2860.

[56] G. Lazarides, Q. Shafi, and C. Wetterich, Proton Lifetime and Fermion Masses in an SO(10) Model, Nucl.Phys. B181 (1981) 287.

[57] R. N. Mohapatra and G. Senjanovic, Neutrino Masses and Mixings in Gauge Models with Spontaneous Parity Violation, Phys.Rev. D23 (1981) 165.

[58] R. Mohapatra and J. Valle, Neutrino Mass and Baryon Number Nonconservation in Superstring Models, Phys.Rev. D34 (1986) 1642.

[59] R. Mohapatra, MECHANISM FOR UNDERSTANDING SMALL NEUTRINO MASS IN SUPERSTRING THEORIES, Phys.Rev.Lett. 56 (1986) 561-563.

[60] R. Foot, H. Lew, X. He, and G. C. Joshi, SEESAW NEUTRINO MASSES INDUCED BY A TRIPLET OF LEPTONS, Z.Phys. C44 (1989) 441.

[61] K. Babu, Model of 'Calculable' Majorana Neutrino Masses, Phys.Lett. B203 (1988) 132.

[62] M. Roncadelli and D. Wyler, NATURALLY LIGHT DIRAC NEUTRINOS IN GAUGE THEORIES, Phys.Lett. B133 (1983) 325.

[63] E. Ma, Pathways to naturally small neutrino masses, Phys.Rev.Lett. 81 (1998) 1171-1174, hep-ph/9805219. 
[64] T. Gherghetta, Dirac neutrino masses with Planck scale lepton number violation, Phys.Rev.Lett. 92 (2004) 161601, hep-ph/0312392.

[65] P.-H. Gu and H.-J. He, Neutrino Mass and Baryon Asymmetry from Dirac Seesaw, JCAP 0612 (2006) 010, hep-ph/0610275.

[66] A. de Gouvea, W.-C. Huang, and J. Jenkins, Pseudo-Dirac Neutrinos in the New Standard Model, Phys.Rev. D80 (2009) 073007, [arXiv:0906.1611].

[67] P. Minkowski, mu $\rightarrow$ e gamma at a rate of one out of 1-billion muon decays?, Phys. Lett. B67 (1977) 421.

[68] M. Gell-Mann, P. Ramond, and R. Slansky in Supergravity, ed. by D. Freedman et al., North Holland (1979).

[69] R. N. Mohapatra and G. Senjanovic, Neutrino mass and spontaneous parity nonconservation, Phys. Rev. Lett. 44 (1980) 912.

[70] T. Yanagida, Horizontal Symmetry and Masses of Neutrinos, Prog.Theor.Phys. 64 (1980) 1103.

[71] K. L. McDonald, Minimal Tree-Level Seesaws with a Heavy Intermediate Fermion, JHEP 1307 (2013) 020, $\operatorname{arXiv:1303.4573.}$

[72] A. Kusenko, F. Takahashi, and T. T. Yanagida, Dark Matter from Split Seesaw, Phys.Lett. B693 (2010) 144-148, [arXiv:1006.1731].

[73] E. Ma, Verifiable radiative seesaw mechanism of neutrino mass and dark matter, Phys.Rev. D73 (2006) 077301, hep-ph/0601225.

[74] L. M. Krauss, S. Nasri, and M. Trodden, A Model for neutrino masses and dark matter, Phys.Rev. D67 (2003) 085002, hep-ph/0210389.

[75] A. de Gouvea, See-saw energy scale and the LSND anomaly, Phys.Rev. D72 (2005) 033005, hep-ph/0501039.

[76] A. Abada, C. Biggio, F. Bonnet, M. Gavela, and T. Hambye, Low energy effects of neutrino masses, JHEP 0712 (2007) 061, arXiv:0707.4058].

[77] A. Palazzo, Phenomenology of light sterile neutrinos: a brief review, Mod. Phys. Lett. A, Vol. 28, No. 7 (2013) 1330004, [arXiv:1302.1102].

[78] LSND Collaboration Collaboration, C. Athanassopoulos et al., Evidence for $\bar{\nu}_{\mu} \rightarrow \bar{\nu}_{e}$ oscillations from the LSND experiment at LAMPF, Phys.Rev.Lett. 77 (1996) 3082-3085, nucl-ex/9605003.

[79] LSND Collaboration Collaboration, A. Aguilar-Arevalo et al., Evidence for neutrino oscillations from the observation of anti-neutrino(electron) appearance in a anti-neutrino(muon) beam, Phys.Rev. D64 (2001) 112007, hep-ex/0104049. 
[80] KARMEN Collaboration Collaboration, B. Armbruster et al., Upper limits for neutrino oscillations $\bar{\nu}_{\mu} \rightarrow \bar{\nu}_{e}$ from muon decay at rest, Phys.Rev. D65 (2002) 112001, hep-ex/0203021.

[81] E. Church, K. Eitel, G. B. Mills, and M. Steidl, Statistical analysis of different $\bar{\nu}_{\mu} \rightarrow \bar{\nu}_{e}$ searches, Phys.Rev. D66 (2002) 013001, hep-ex/0203023.

[82] MiniBooNE Collaboration Collaboration, A. Aguilar-Arevalo et al., A Combined $\nu_{\mu} \rightarrow \nu_{e}$ and $\bar{\nu}_{\mu} \rightarrow \bar{\nu}_{e}$ Oscillation Analysis of the MiniBooNE Excesses, arXiv:1207.4809.

[83] M. Antonello, B. Baibussinov, P. Benetti, E. Calligarich, N. Canci, et al., Experimental search for the LSND anomaly with the ICARUS LAr TPC detector in the CNGS beam, arXiv:1209.0122.

[84] J. Kopp, P. A. N. Machado, M. Maltoni, and T. Schwetz, Sterile Neutrino Oscillations: The Global Picture, arXiv:1303.3011.

[85] G. Mention, M. Fechner, T. Lasserre, T. Mueller, D. Lhuillier, et al., The Reactor Antineutrino Anomaly, Phys.Rev. D83 (2011) 073006, [arXiv:1101.2755].

[86] T. Mueller, D. Lhuillier, M. Fallot, A. Letourneau, S. Cormon, et al., Improved Predictions of Reactor Antineutrino Spectra, Phys.Rev. C83 (2011) 054615, [arXiv:1101.2663].

[87] P. Huber, On the determination of anti-neutrino spectra from nuclear reactors, Phys.Rev. C84 (2011) 024617, arXiv:1106.0687.

[88] A. Hayes, J. Friar, G. Garvey, and G. Jonkmans, Reanalysis of the Reactor Neutrino Anomaly, arXiv:1309.4146.

[89] F. Von Feilitzsch, A. Hahn, and K. Schreckenbach, EXPERIMENTAL BETA SPECTRA FROM PU-239 AND U-235 THERMAL NEUTRON FISSION PRODUCTS AND THEIR CORRELATED ANTI-NEUTRINOS SPECTRA, Phys.Lett. B118 (1982) $162-166$.

[90] A. Hahn, K. Schreckenbach, G. Colvin, B. Krusche, W. Gelletly, et al., ANTI-NEUTRINO SPECTRA FROM PU-241 AND PU-239 THERMAL NEUTRON FISSION PRODUCTS, Phys.Lett. B218 (1989) 365-368.

[91] J. Abdurashitov, V. Gavrin, S. Girin, V. Gorbachev, P. Gurkina, et al., Measurement of the response of a Ga solar neutrino experiment to neutrinos from an Ar-37 source, Phys.Rev. C73 (2006) 045805, nucl-ex/0512041.

[92] C. Giunti, M. Laveder, Y. Li, Q. Liu, and H. Long, Update of Short-Baseline Electron Neutrino and Antineutrino Disappearance, Phys.Rev. D86 (2012) 113014, arXiv:1210.5715].

[93] J. Conrad, C. Ignarra, G. Karagiorgi, M. Shaevitz, and J. Spitz, Sterile Neutrino Fits to Short Baseline Neutrino Oscillation Measurements, arXiv:1207.4765.

[94] M. Archidiacono, N. Fornengo, C. Giunti, S. Hannestad, and A. Melchiorri, Sterile Neutrinos: Cosmology vs Short-BaseLine Experiments, arXiv:1302.6720. 
[95] S. K. Agarwalla and P. Huber, LSND reloaded, Phys.Lett. B696 (2011) 359-361, arXiv: 1007.3228].

[96] A. de Gouvea and W.-C. Huang, Constraining the (Low-Energy) Type-I Seesaw, Phys.Rev. D85 (2012) 053006, arXiv:1110.6122.

[97] C. Rubbia, A. Guglielmi, F. Pietropaolo, and P. Sala, Sterile neutrinos: the necessity for a 5 sigma definitive clarification, arXiv: 1304.2047

[98] on behalf of NESSiE Collaboration Collaboration, U. Kose, NESSiE: The Experimental Sterile Neutrino Search in Short-Base-Line at CERN, arXiv:1304.7127.

[99] S. M. Bilenky, S. Petcov, and B. Pontecorvo, Lepton Mixing, $\mu \rightarrow e+\gamma$ Decay and Neutrino Oscillations, Phys.Lett. B67 (1977) 309.

[100] T. Cheng and L.-F. Li, $\mu \rightarrow e \gamma$ IN THEORIES WITH DIRAC AND MAJORANA NEUTRINO MASS TERMS, Phys.Rev.Lett. 45 (1980) 1908.

[101] S. M. Bilenky and S. Petcov, Massive Neutrinos and Neutrino Oscillations, Rev.Mod.Phys. 59 (1987) 671.

[102] J. Casas and A. Ibarra, Oscillating neutrinos and muon to e, gamma, Nucl.Phys. B618 (2001) 171-204, hep-ph/0103065.

[103] S. Lavignac, I. Masina, and C. A. Savoy, $\tau \rightarrow \mu \gamma$ and $\mu \rightarrow e \gamma$ as probes of neutrino mass models, Phys.Lett. B520 (2001) 269-278, hep-ph/0106245.

[104] S. Pascoli, S. Petcov, and C. Yaguna, Quasidegenerate neutrino mass spectrum, $\mu \rightarrow e+\gamma$ decay and leptogenesis, Phys.Lett. B564 (2003) 241-254, hep-ph/0301095.

[105] A. Ibarra and G. G. Ross, Neutrino phenomenology: The Case of two right-handed neutrinos, Phys.Lett. B591 (2004) 285-296, hep-ph/0312138.

[106] A. Ibarra, E. Molinaro, and S. Petcov, Low Energy Signatures of the TeV Scale See-Saw Mechanism, Phys.Rev. D84 (2011) 013005, [arXiv:1103.6217.

[107] L. Lello and D. Boyanovsky, Charged lepton mixing via heavy sterile neutrinos, arXiv:1212.4167.

[108] A. Abada, D. Das, A. Teixeira, A. Vicente, and C. Weiland, Tree-level lepton universality violation in the presence of sterile neutrinos: impact for $R_{K}$ and $R_{\pi}$, JHEP 1302 (2013) 048, arXiv:1211.3052].

[109] R. Alonso, M. Dhen, M. Gavela, and T. Hambye, Muon conversion to electron in nuclei in type-I seesaw models, JHEP 1301 (2013) 118, [arXiv:1209.2679].

[110] S. Antusch, C. Biggio, E. Fernandez-Martinez, M. Gavela, and J. Lopez-Pavon, Unitarity of the Leptonic Mixing Matrix, JHEP 0610 (2006) 084, hep-ph/0607020.

[111] D. Forero, S. Morisi, M. Tortola, and J. Valle, Lepton flavor violation and non-unitary lepton mixing in low-scale type-I seesaw, JHEP 1109 (2011) 142, arXiv:1107.6009. 
[112] COMET Collaboration Collaboration, E. V. Hungerford, COMET/PRISM muon to electron conversion at J-PARC, AIP Conf.Proc. 1182 (2009) 694-697.

[113] COMET Collaboration Collaboration, Y. Cui et al., Conceptual design report for experimental search for lepton flavor violating mu- - e-conversion at sensitivity of $10^{* *}(-16)$ with a slow-extracted bunched proton beam (COMET), .

[114] Mu2e Collaboration Collaboration, R. Abrams et al., Mu2e Conceptual Design Report, arXiv:1211.7019.

[115] D. Wyler and L. Wolfenstein, Massless Neutrinos in Left-Right Symmetric Models, Nucl.Phys. B218 (1983) 205.

[116] M. Gonzalez-Garcia and J. Valle, FAST DECAYING NEUTRINOS AND OBSERVABLE FLAVOR VIOLATION IN A NEW CLASS OF MAJORON MODELS, Phys.Lett. B216 (1989) 360.

[117] G. Branco, W. Grimus, and L. Lavoura, THE SEESAW MECHANISM IN THE PRESENCE OF A CONSERVED LEPTON NUMBER, Nucl.Phys. B312 (1989) 492.

[118] J. Kersten and A. Y. Smirnov, Right-Handed Neutrinos at CERN LHC and the Mechanism of Neutrino Mass Generation, Phys.Rev. D76 (2007) 073005, [arXiv:0705.3221].

[119] M. Shaposhnikov, A possible symmetry of the $\nu m s m$, Nucl. Phys. B763 (2007) 49-59, hep-ph/0605047.

[120] M. Gavela, T. Hambye, D. Hernandez, and P. Hernandez, Minimal Flavour Seesaw Models, JHEP 0909 (2009) 038, [arXiv:0906.1461].

[121] S. Blanchet, T. Hambye, and F.-X. Josse-Michaux, Reconciling leptogenesis with observable $\mu \rightarrow$ e r rates, JHEP 1004 (2010) 023, arXiv:0912.3153.

[122] M. Duerr, D. P. George, and K. L. McDonald, Neutrino Mass and $\mu \rightarrow e+\gamma$ from a Mini-Seesaw, JHEP 1107 (2011) 103, arXiv:1105.0593.

[123] K. L. McDonald, Light Neutrinos from a Mini-Seesaw Mechanism in Warped Space, Phys.Lett. B696 (2011) 266-272, arXiv:1010.2659.

[124] J. Barry and W. Rodejohann, Lepton number and lepton flavour violation in left-right symmetric theories, arXiv:1303.6324

[125] W. Rodejohann, Neutrino-less Double Beta Decay and Particle Physics, Int.J.Mod.Phys. E20 (2011) 1833-1930, arXiv:1106.1334.

[126] S. Bilenky and C. Giunti, Neutrinoless double-beta decay: A brief review, Mod.Phys.Lett. A27 (2012) 1230015, arXiv:1203.5250.

[127] M. Blennow, E. Fernandez-Martinez, J. Lopez-Pavon, and J. Menendez, Neutrinoless double beta decay in seesaw models, JHEP 1007 (2010) 096, arXiv:1005.3240]. 
[128] J. Barry, W. Rodejohann, and H. Zhang, Sterile Neutrinos for Warm Dark Matter and the Reactor Anomaly in Flavor Symmetry Models, JCAP 1201 (2012) 052, arXiv:1110.6382.

[129] M. Mitra, G. Senjanovic, and F. Vissani, Neutrinoless Double Beta Decay and Heavy Sterile Neutrinos, Nucl.Phys. B856 (2012) 26-73, [arXiv:1108.0004].

[130] M. Mitra, G. Senjanovic, and F. Vissani, Heavy Sterile Neutrinos and Neutrinoless Double Beta Decay, arXiv:1205.3867.

[131] J. Lopez-Pavon, S. Pascoli, and C.-f. Wong, Can heavy neutrinos dominate neutrinoless double beta decay?, arXiv:1209.5342.

[132] F. Bezrukov, nu MSM-predictions for neutrinoless double beta decay, Phys.Rev. D72 (2005) 071303, hep-ph/0505247.

[133] T. Asaka, S. Eijima, and H. Ishida, Mixing of Active and Sterile Neutrinos, JHEP 1104 (2011) 011, arXiv:1101.1382.

[134] T. Asaka and S. Eijima, Direct Search for Right-handed Neutrinos and Neutrinoless Double Beta Decay, arXiv:1308.3550.

[135] I. Girardi, A. Meroni, and S. Petcov, Neutrinoless Double Beta Decay in the Presence of Light Sterile Neutrinos, arXiv:1308.5802.

[136] A. Merle and V. Niro, Influence of a keV sterile neutrino on neutrino-less double beta decay - how things changed in the recent years, arXiv:1302.2032.

[137] P. Bamert, C. Burgess, and R. Mohapatra, Heavy sterile neutrinos and neutrinoless double beta decay, Nucl.Phys. B438 (1995) 3-16, hep-ph/9408367.

[138] P. Benes, A. Faessler, F. Simkovic, and S. Kovalenko, Sterile neutrinos in neutrinoless double beta decay, Phys.Rev. D71 (2005) 077901, hep-ph/0501295.

[139] H. Klapdor-Kleingrothaus, I. Krivosheina, A. Dietz, and O. Chkvorets, Search for neutrinoless double beta decay with enriched Ge-76 in Gran Sasso 1990-2003, Phys.Lett. B586 (2004) 198-212, hep-ph/0404088.

[140] H. Klapdor-Kleingrothaus and I. Krivosheina, The evidence for the observation of Onu beta beta decay: The identification of Onu beta beta events from the full spectra, Mod.Phys.Lett. A21 (2006) 1547-1566.

[141] G. Fogli, E. Lisi, A. Marrone, A. Melchiorri, A. Palazzo, et al., Observables sensitive to absolute neutrino masses. 2., Phys.Rev. D78 (2008) 033010, arXiv:0805.2517.

[142] J. Bergstrm, Combining and comparing neutrinoless double beta decay experiments using different nuclei, JHEP 1302 (2013) 093, arXiv:1212.4484].

[143] P. S. B. Dev, S. Goswami, M. Mitra, and W. Rodejohann, Constraining Neutrino Mass from Neutrinoless Double Beta Decay, arXiv:1305.0056. 
[144] A. de Gouvea and P. Vogel, Lepton Flavor and Number Conservation, and Physics Beyond the Standard Model, arXiv:1303.4097.

[145] B. Schwingenheuer, Status and prospects of searches for neutrinoless double beta decay, arXiv:1210.7432.

[146] A. Atre, T. Han, S. Pascoli, and B. Zhang, The Search for Heavy Majorana Neutrinos, JHEP 0905 (2009) 030, arXiv:0901.3589.

[147] D. Gorbunov and M. Shaposhnikov, How to find neutral leptons of the $\nu M S M$ ?, JHEP 10 (2007) 015, arXiv:0705.1729.

[148] O. Ruchayskiy and A. Ivashko, Experimental bounds on sterile neutrino mixing angles, JHEP 1206 (2012) 100, arXiv:1112.3319.

[149] D. Gorbunov and M. Shaposhnikov, Search for GeV-scale sterile neutrinos responsible for active neutrino masses and baryon asymmetry of the Universe, Contribution to Open Symposium - European Strategy Preparatory Group, Krakow, Poland, September 2012, https://indico.cern.ch/contributionDisplay.py?contribId=17EconfId=175067 (2012).

[150] L. Lello and D. Boyanovsky, Searching for sterile neutrinos from $\pi$ and $K$ decays, arXiv:1208.5559.

[151] T. Asaka, S. Eijima, and A. Watanabe, Heavy neutrino search in accelerator-based experiments, arXiv:1212.1062.

[152] S. Gninenko, D. Gorbunov, and M. Shaposhnikov, Search for GeV-scale sterile neutrinos responsible for active neutrino oscillations and baryon asymmetry of the Universe, Adv.High Energy Phys. 2012 (2012) 718259, arXiv:1301.5516].

[153] N. Quintero, G. Lopez Castro, and D. Delepine, Lepton number violation in top quark and neutral B meson decays, Phys.Rev. D84 (2011) 096011, [arXiv:1108.6009].

[154] J. C. Helo, S. Kovalenko, and I. Schmidt, On sterile neutrino mixing with $\nu_{\tau}$, Phys.Rev. D84 (2011) 053008, arXiv:1105.3019.

[155] J. C. Helo, S. Kovalenko, and I. Schmidt, Sterile neutrinos in lepton number and lepton flavor violating decays, Nucl.Phys. B853 (2011) 80-104, [arXiv:1005.1607].

[156] G. Lopez Castro and N. Quintero, Lepton number violating four-body tau lepton decays, Phys.Rev. D85 (2012) 076006, arXiv:1203.0537.

[157] L3 Collaboration, P. Achard et al., Search for heavy neutral and charged leptons in $e^{+} e^{-}$ annihilation at LEP, Phys. Lett. B517 (2001) 75-85, hep-ex/0107015.

[158] T. Yamazaki et al., Search for heavy neutrinos in kaon decay, . IN *LEIPZIG 1984, Proceedings, High Energy Physics, Vol. 1*, 262.

[159] M. Daum et al., The KARMEN Time Anomaly: Search for a Neutral Particle of Mass 33.9 MeV in Pion Decay, Phys. Rev. Lett. 85 (2000) 1815-1818, hep-ex/0008014.

[160] G. Bernardi et al., Search for neutrino decay, Phys. Lett. B166 (1986) 479. 
[161] G. Bernardi et al., Further limits on heavy neutrino couplings, Phys. Lett. B203 (1988) 332 .

[162] NuTeV Collaboration, A. Vaitaitis et al., Search for neutral heavy leptons in a high-energy neutrino beam, Phys. Rev. Lett. 83 (1999) 4943-4946, hep-ex/9908011.

[163] CHARM Collaboration, F. Bergsma et al., A search for decays of heavy neutrinos in the mass range 0.5 GeV to 2.8 GeV, Phys. Lett. B166 (1986) 473.

[164] NOMAD Collaboration, P. Astier et al., Search for heavy neutrinos mixing with tau neutrinos, Phys. Lett. B506 (2001) 27-38, hep-ex/0101041.

[165] WA66 Collaboration, A. M. Cooper-Sarkar et al., Search for heavy neutrino decays in the BEBC beam dump experiment, Phys. Lett. B160 (1985) 207.

[166] I. M. Shoemaker, K. Petraki, and A. Kusenko, Collider signatures of sterile neutrinos in models with a gauge-singlet Higgs, JHEP 1009 (2010) 060, arXiv:1006.5458.

[167] A. Datta, M. Guchait, and A. Pilaftsis, Probing lepton number violation via majorana neutrinos at hadron supercolliders, Phys.Rev. D50 (1994) 3195-3203, hep-ph/9311257.

[168] J. Almeida, F.M.L., Y. D. A. Coutinho, J. A. Martins Simoes, P. Queiroz Filho, and C. Porto, Same sign dileptons as a signature for heavy Majorana neutrinos in hadron hadron collisions, Phys.Lett. B400 (1997) 331-334, hep-ph/9703441.

[169] J. Almeida, F.M.L., Y. D. A. Coutinho, J. A. Martins Simoes, and M. do Vale, On a signature for heavy Majorana neutrinos in hadronic collisions, Phys.Rev. D62 (2000) 075004, hep-ph/0002024.

[170] O. Panella, M. Cannoni, C. Carimalo, and Y. Srivastava, Signals of heavy Majorana neutrinos at hadron colliders, Phys.Rev. D65 (2002) 035005, hep-ph/0107308.

[171] K. Cheung and O. Seto, Phenomenology of TeV right-handed neutrino and the dark matter model, Phys.Rev. D69 (2004) 113009, hep-ph/0403003.

[172] T. Han and B. Zhang, Signatures for Majorana neutrinos at hadron colliders, Phys.Rev.Lett. 97 (2006) 171804, hep-ph/0604064.

[173] F. del Aguila, J. Aguilar-Saavedra, and R. Pittau, Heavy neutrino signals at large hadron colliders, JHEP 0710 (2007) 047, hep-ph/0703261.

[174] K. Huitu, S. Khalil, H. Okada, and S. K. Rai, Signatures for right-handed neutrinos at the Large Hadron Collider, Phys.Rev.Lett. 101 (2008) 181802, [arXiv:0803.2799].

[175] S. Kovalenko, Z. Lu, and I. Schmidt, Lepton Number Violating Processes Mediated by Majorana Neutrinos at Hadron Colliders, Phys.Rev. D80 (2009) 073014, arXiv:0907.2533.

[176] S. Matsumoto, T. Nabeshima, and K. Yoshioka, Seesaw Neutrino Signals at the Large Hadron Collider, JHEP 1006 (2010) 058, arXiv:1004.3852. 
[177] M. Aoki and S. Kanemura, Probing the Majorana nature of TeV-scale radiative seesaw models at collider experiments, Phys.Lett. B689 (2010) 28-35, [arXiv:1001.0092].

[178] A. Das and N. Okada, Inverse Seesaw Neutrino Signatures at LHC and ILC, arXiv:1207.3734.

[179] I. Baldes, N. F. Bell, K. Petraki, and R. R. Volkas, Two radiative inverse seesaw models, dark matter, and baryogenesis, arXiv:1304.6162.

[180] D. Dinh and S. Petcov, Lepton Flavor Violating $\tau$ Decays in TeV Scale Type I See-Saw and Higgs Triplet Models, arXiv:1308.4311.

[181] P. S. B. Dev, A. Pilaftsis, and U.-k. Yang, New Production Mechanism for Heavy Neutrinos at the LHC, arXiv:1308.2209.

[182] A. Dias, C. de S. Pires, and P. R. da Silva, How the Inverse See-Saw Mechanism Can Reveal Itself Natural, Canonical and Independent of the Right-Handed Neutrino Mass, Phys.Rev. D84 (2011) 053011, arXiv:1107.0739].

[183] N. Haba, S. Matsumoto, and K. Yoshioka, Observable Seesaw and its Collider Signatures, Phys.Lett. B677 (2009) 291-295, arXiv:0901.4596].

[184] J. C. Pati and A. Salam, Lepton Number as the Fourth Color, Phys.Rev. D10 (1974) $275-289$.

[185] R. N. Mohapatra and J. C. Pati, Left-Right Gauge Symmetry and an Isoconjugate Model of CP Violation, Phys.Rev. D11 (1975) 566-571.

[186] G. Senjanovic and R. N. Mohapatra, Exact Left-Right Symmetry and Spontaneous Violation of Parity, Phys.Rev. D12 (1975) 1502.

[187] W.-Y. Keung and G. Senjanovic, MAJORANA NEUTRINOS AND THE PRODUCTION OF THE RIGHT-HANDED CHARGED GAUGE BOSON, Phys.Rev.Lett. 50 (1983) 1427.

[188] A. Maiezza, M. Nemevsek, F. Nesti, and G. Senjanovic, Left-Right Symmetry at LHC, Phys.Rev. D82 (2010) 055022, [arXiv:1005.5160].

[189] J. Aguilar-Saavedra, F. Deppisch, O. Kittel, and J. Valle, Flavour in heavy neutrino searches at the LHC, Phys.Rev. D85 (2012) 091301, arXiv:1203.5998.

[190] M. Nemevsek, G. Senjanovic, and V. Tello, Left-Right Symmetry: from Majorana to Dirac, arXiv:1211.2837.

[191] C.-Y. Chen, P. S. B. Dev, and R. Mohapatra, Probing Heavy-Light Neutrino Mixing in Left-Right Seesaw Models at the LHC, Phys.Rev. D88 (2013) 033014, [arXiv:1306.2342].

[192] CMS Collaboration Collaboration, S. Chatrchyan et al., Search for heavy neutrinos and $W[R]$ bosons with right-handed couplings in a left-right symmetric model in pp collisions at sqrt(s) = 7 TeV, Phys.Rev.Lett. 109 (2012) 261802, arXiv:1210.2402. 
[193] ATLAS Collaboration Collaboration, G. Aad et al., Search for heavy neutrinos and right-handed $W$ bosons in events with two leptons and jets in pp collisions at $\sqrt{s}=7$ TeV with the ATLAS detector, Eur.Phys.J. C72 (2012) 2056, arXiv:1203.5420].

[194] F. Deppisch, H. Pas, A. Redelbach, R. Ruckl, and Y. Shimizu, The SUSY seesaw model and lepton flavor violation at a future electron positron linear collider, Phys.Rev. D69 (2004) 054014, hep-ph/0310053.

[195] C. Cheung, L. J. Hall, and D. Pinner, Seesaw Spectroscopy at Colliders, arXiv:1103.3520.

[196] S. Heinemeyer, M. Herrero, S. Penaranda, and A. Rodriguez-Sanchez, Higgs Boson Masses in the MSSM with Heavy Majorana Neutrinos, JHEP 1105 (2011) 063, arXiv:1007.5512].

[197] I. Gogoladze, B. He, and Q. Shafi, Inverse Seesaw in NMSSM and 126 GeV Higgs Boson, Phys.Lett. B718 (2013) 1008-1013, [arXiv:1209.5984].

[198] W. Wang, J. M. Yang, and L. L. You, Higgs boson mass in NMSSM with right-handed neutrino, arXiv:1303.6465.

[199] P. Draper and H. E. Haber, Decoupling of the Right-handed Neutrino Contribution to the Higgs Mass in Supersymmetric Models, arXiv:1304.6103.

[200] S. King and T. Yanagida, Testing the see-saw mechanism at collider energies, Prog.Theor.Phys. 114 (2006) 1035-1043, hep-ph/0411030.

[201] L. A. Anchordoqui, H. Goldberg, and G. Steigman, Right-Handed Neutrinos as the Dark Radiation: Status and Forecasts for the LHC, Phys.Lett. B718 (2013) 1162-1165, [arXiv:1211.0186].

[202] S. Blanchet, Z. Chacko, S. S. Granor, and R. N. Mohapatra, Probing Resonant Leptogenesis at the LHC, Phys.Rev. D82 (2010) 076008, [arXiv:0904.2174].

[203] A. Boyarsky, O. Ruchayskiy, and M. Shaposhnikov, The role of sterile neutrinos in cosmology and astrophysics, Ann. Rev. Nucl. Part. Sci. 59 (2009) 191-214, arXiv:0901.0011.

[204] A. Merle, Constraining models for keV sterile neutrinos by quasi-degenerate active neutrinos, Phys.Rev. D86 (2012) 121701, [arXiv:1210.6036].

[205] Y. Li and Z.-z. Xing, Possible Capture of keV Sterile Neutrino Dark Matter on Radioactive $\beta$-decaying Nuclei, Phys.Lett. B695 (2011) 205-210, [arXiv:1009.5870].

[206] W. Liao, keV scale $\nu_{R}$ dark matter and its detection in $\beta$ decay experiment, Phys.Rev. D82 (2010) 073001, arXiv:1005.3351.

[207] S. Ando and A. Kusenko, Interactions of keV sterile neutrinos with matter, Phys.Rev. D81 (2010) 113006, arXiv:1001.5273.

[208] A. de Gouvea, J. Jenkins, and N. Vasudevan, Neutrino Phenomenology of Very Low-Energy Seesaws, Phys.Rev. D75 (2007) 013003, hep-ph/0608147. 
[209] J. Formaggio and J. Barrett, Resolving the Reactor Neutrino Anomaly with the KATRIN Neutrino Experiment, Phys.Lett. B706 (2011) 68-71, [arXiv:1105.1326].

[210] C. Kraus, A. Singer, K. Valerius, and C. Weinheimer, Limit on sterile neutrino contribution from the Mainz Neutrino Mass Experiment, arXiv:1210.4194.

[211] A. Esmaili and O. L. Peres, KATRIN Sensitivity to Sterile Neutrino Mass in the Shadow of Lightest Neutrino Mass, Phys.Rev. D85 (2012) 117301, [arXiv:1203.2632.

[212] T. Asaka and A. Watanabe, Atmospheric Sterile Neutrinos, JHEP 1207 (2012) 112, arXiv:1202.0725.

[213] H. Nunokawa, O. Peres, and R. Zukanovich Funchal, Probing the LSND mass scale and four neutrino scenarios with a neutrino telescope, Phys.Lett. B562 (2003) 279-290, hep-ph/0302039.

[214] S. Choubey, Signature of sterile species in atmospheric neutrino data at neutrino telescopes, JHEP 0712 (2007) 014, [arXiv:0709.1937].

[215] A. Esmaili, F. Halzen, and O. Peres, Constraining Sterile Neutrinos with AMANDA and IceCube Atmospheric Neutrino Data, JCAP 1211 (2012) 041, [arXiv:1206.6903.

[216] A. Esmaili, F. Halzen, and O. Peres, Exploring $\nu_{\tau}-\nu_{s}$ mixing with cascade events in DeepCore, arXiv:1303.3294.

[217] A. Esmaili and A. Y. Smirnov, Restricting the LSND and MiniBooNE sterile neutrinos with the IceCube atmospheric neutrino data, arXiv:1307.6824.

[218] A. Gutlein, C. Ciemniak, F. von Feilitzsch, N. Haag, M. Hofmann, et al., Solar and atmospheric neutrinos: Background sources for the direct dark matter search, Astropart.Phys. 34 (2010) 90-96, [arXiv:1003.5530].

[219] F. Bezrukov and M. Shaposhnikov, Searching for dark matter sterile neutrino in laboratory, Phys.Rev. D75 (2007) 053005, hep-ph/0611352.

[220] H. de Vega, O. Moreno, E. M. de Guerra, M. R. Medrano, and N. Sanchez, Role of sterile neutrino warm dark matter in rhenium and tritium beta decays, Nucl.Phys. B866 (2013) 177-195, arXiv:1109.3452].

[221] E. Akhmedov, A. Kartavtsev, M. Lindner, L. Michaels, and J. Smirnov, Improving Electro-Weak Fits with TeV-scale Sterile Neutrinos, arXiv:1302.1872.

[222] V. Hoang, P. Q. Hung, and A. Kamat, Electroweak precision constraints on the electroweak-scale right-handed neutrino model, arXiv:1303.0428.

[223] G. Giudice, A. Notari, M. Raidal, A. Riotto, and A. Strumia, Towards a complete theory of thermal leptogenesis in the SM and MSSM, Nucl.Phys. B685 (2004) 89-149, hep-ph/0310123.

[224] B. Garbrecht, Leptogenesis: The Other Cuts, Nucl.Phys. B847 (2011) 350-366, arXiv: 1011.3122]. 
[225] A. Anisimov, D. Besak, and D. Bodeker, Thermal production of relativistic Majorana neutrinos: Strong enhancement by multiple soft scattering, JCAP 1103 (2011) 042, arXiv:1012.3784.

[226] V. Mukhanov, Physical foundations of cosmology, Cambridge University Press (2005).

[227] S. Weinberg, Cosmology, Oxford University Press (2008).

[228] G. Mangano, G. Miele, S. Pastor, T. Pinto, O. Pisanti, et al., Relic neutrino decoupling including flavor oscillations, Nucl.Phys. B729 (2005) 221-234, hep-ph/0506164.

[229] V. Shvartsman, Density of relict particles with zero rest mass in the universe, Pisma Zh.Eksp.Teor.Fiz. 9 (1969) 315-317.

[230] G. Steigman, D. Schramm, and J. Gunn, Cosmological Limits to the Number of Massive Leptons, Phys.Lett. B66 (1977) 202-204.

[231] A. Dolgov, Neutrinos in cosmology, Phys.Rept. 370 (2002) 333-535, hep-ph/0202122.

[232] J. Lesgourgues and S. Pastor, Neutrino mass from Cosmology, Adv.High Energy Phys. 2012 (2012) 608515, arXiv:1212.6154.

[233] Y. Zeldovich and M. Y. Khlopov, THE NEUTRINO MASS IN ELEMENTARY PARTICLE PHYSICS AND IN BIG BANG COSMOLOGY, Sov.Phys.Usp. 24 (1981) $755-774$.

[234] C. Boehm, M. J. Dolan, and C. McCabe, Increasing Neff with particles in thermal equilibrium with neutrinos, JCAP 1212 (2012) 027, [arXiv:1207.0497].

[235] G. Steigman, Equivalent Neutrinos, Light WIMPs, and the Chimera of Dark Radiation, arXiv: 1303.0049 .

[236] C. Boehm, M. J. Dolan, and C. McCabe, A Lower Bound on the Mass of Cold Thermal Dark Matter from Planck, arXiv:1303.6270.

[237] G. Hinshaw, D. Larson, E. Komatsu, D. Spergel, C. Bennett, et al., Nine-Year Wilkinson Microwave Anisotropy Probe (WMAP) Observations: Cosmological Parameter Results, arXiv:1212.5226.

[238] A. A. Starobinsky, A new type of isotropic cosmological models without singularity, Phys. Lett. B91 (1980) 99-102.

[239] A. H. Guth, The inflationary universe: A possible solution to the horizon and flatness problems, Phys. Rev. D23 (1981) 347-356.

[240] A. Dolgov and A. D. Linde, Baryon Asymmetry in Inflationary Universe, Phys.Lett. B116 (1982) 329.

[241] L. Kofman, A. D. Linde, and A. A. Starobinsky, Reheating after inflation, Phys.Rev.Lett. 73 (1994) 3195-3198, hep-th/9405187. 
[242] L. Kofman, A. D. Linde, and A. A. Starobinsky, Towards the theory of reheating after inflation, Phys. Rev. D56 (1997) 3258-3295, hep-ph/9704452.

[243] M. Drewes and J. U. Kang, The Kinematics of Cosmic Reheating, Nucl. Phys. B 875 (2013) 315-350, [arXiv:1305.0267].

[244] V. F. Mukhanov and G. Chibisov, Quantum Fluctuation and Nonsingular Universe. (In Russian), JETP Lett. 33 (1981) 532-535.

[245] L. Canetti, M. Drewes, and M. Shaposhnikov, Matter and Antimatter in the Universe, New J. Phys. 14 (2012) 095012, [arXiv:1204.4186].

[246] V. A. Kuzmin, V. A. Rubakov, and M. E. Shaposhnikov, On the anomalous electroweak baryon number nonconservation in the early universe, Phys. Lett. B155 (1985) 36.

[247] S. L. Adler, Axial vector vertex in spinor electrodynamics, Phys.Rev. 177 (1969) $2426-2438$.

[248] J. Bell and R. Jackiw, A PCAC puzzle: $p i^{0} \rightarrow \gamma \gamma$ in the sigma model, Nuovo Cim. A60 (1969) 47-61.

[249] G. 't Hooft, Symmetry Breaking Through Bell-Jackiw Anomalies, Phys.Rev.Lett. 37 (1976) 8-11.

[250] L. Canetti, M. Drewes, and M. Shaposhnikov, Sterile Neutrinos as the Origin of Dark and Baryonic Matter, Phys. Rev. Lett. 110 (2013) 061801, [arXiv:1204.3902].

[251] L. Canetti, M. Drewes, T. Frossard, and M. Shaposhnikov, Dark Matter, Baryogenesis and Neutrino Oscillations from Right Handed Neutrinos, Phys.Rev. D87 (2012) 093006, [arXiv:1208.4607].

[252] D. J. Schwarz and M. Stuke, Lepton asymmetry and the cosmic QCD transition, JCAP 0911 (2009) 025, arXiv:0906.3434.

[253] M. Laine and M. Shaposhnikov, Sterile neutrino dark matter as a consequence of $\nu M S M$ induced lepton asymmetry, JCAP 0806 (2008) 031, arXiv:0804.4543.

[254] C. T. Kishimoto and G. M. Fuller, Lepton Number-Driven Sterile Neutrino Production in the Early Universe, Phys.Rev. D78 (2008) 023524, [arXiv:0802.3377].

[255] R. Foot, M. J. Thomson, and R. Volkas, Large neutrino asymmetries from neutrino oscillations, Phys.Rev. D53 (1996) 5349-5353, hep-ph/9509327].

[256] H.-S. Kang and G. Steigman, Cosmological constraints on neutrino degeneracy, Nucl.Phys. B372 (1992) 494-520.

[257] A. Dolgov, S. Hansen, S. Pastor, S. Petcov, G. Raffelt, et al., Cosmological bounds on neutrino degeneracy improved by flavor oscillations, Nucl.Phys. B632 (2002) 363-382, hep-ph/0201287.

[258] Y. Y. Wong, Analytical treatment of neutrino asymmetry equilibration from flavor oscillations in the early universe, Phys.Rev. D66 (2002) 025015, hep-ph/0203180. 
[259] K. N. Abazajian, J. F. Beacom, and N. F. Bell, Stringent constraints on cosmological neutrino anti-neutrino asymmetries from synchronized flavor transformation, Phys.Rev. D66 (2002) 013008, astro-ph/0203442.

[260] G. Mangano, G. Miele, S. Pastor, O. Pisanti, and S. Sarikas, Updated BBN bounds on the cosmological lepton asymmetry for non-zero $\theta_{13}$, Phys.Lett. B708 (2012) 1-5, arXiv:1110.4335.

[261] P. D. Serpico and G. G. Raffelt, Lepton asymmetry and primordial nucleosynthesis in the era of precision cosmology, Phys.Rev. D71 (2005) 127301, astro-ph/0506162.

[262] S. Pastor, T. Pinto, and G. G. Raffelt, Relic density of neutrinos with primordial asymmetries, Phys.Rev.Lett. 102 (2009) 241302, arXiv:0808.3137.

[263] G. Mangano, G. Miele, S. Pastor, O. Pisanti, and S. Sarikas, Constraining the cosmic radiation density due to lepton number with Big Bang Nucleosynthesis, JCAP 1103 (2011) 035, arXiv:1011.0916.

[264] E. Castorina, U. Franca, M. Lattanzi, J. Lesgourgues, G. Mangano, et al., Cosmological lepton asymmetry with a nonzero mixing angle $\theta_{13}$, Phys.Rev. D86 (2012) 023517, arXiv:1204.2510].

[265] G. Gamow, Expanding universe and the origin of elements, Phys.Rev. 70 (1946) 572-573.

[266] O. Ruchayskiy and A. Ivashko, Restrictions on the lifetime of sterile neutrinos from primordial nucleosynthesis, arXiv:1202.2841.

[267] R. Barbieri and A. Dolgov, Bounds on Sterile-neutrinos from Nucleosynthesis, Phys.Lett. B237 (1990) 440.

[268] K. Kainulainen, LIGHT SINGLET NEUTRINOS AND THE PRIMORDIAL NUCLEOSYNTHESIS, Phys.Lett. B244 (1990) 191-195.

[269] J. Lesgourgues and S. Pastor, Massive neutrinos and cosmology, Phys.Rept. 429 (2006) 307-379, astro-ph/0603494.

[270] G. Steigman, Neutrinos And Big Bang Nucleosynthesis, Adv.High Energy Phys. 2012 (2012) 268321, arXiv:1208.0032.

[271] C. Brust, D. E. Kaplan, and M. T. Walters, New Light Species and the CMB, arXiv:1303.5379.

[272] M. Y. Khlopov and S. Petcov, Possible cosmological effect of CP violation in neutrino oscillations, Phys.Lett. B99 (1981) 117.

[273] P. Di Bari, Update on neutrino mixing in the early universe, Phys.Rev. D65 (2002) 043509, hep-ph/0108182.

[274] G. Steigman, Primordial Nucleosynthesis: The Predicted and Observed Abundances and Their Consequences, arXiv:1008.4765. 
[275] G. Mangano and P. D. Serpico, A robust upper limit on $N_{\text {eff }}$ from BBN, circa 2011, Phys.Lett. B701 (2011) 296-299, arXiv:1103.1261.

[276] M. Pettini and R. Cooke, A new, precise measurement of the primordial abundance of Deuterium, Monthly Notices of the Royal Astronomical Society 425 (2012) 24772486, arXiv:1205.3785.

[277] K. M. Nollett and G. P. Holder, An analysis of constraints on relativistic species from primordial nucleosynthesis and the cosmic microwave background, arXiv:1112.2683.

[278] J. Hamann, S. Hannestad, G. G. Raffelt, and Y. Y. Wong, Sterile neutrinos with eV masses in cosmology: How disfavoured exactly?, JCAP 1109 (2011) 034, arXiv:1108.4136].

[279] M. Drewes, S. Mendizabal, and C. Weniger, The Boltzmann Equation from Quantum Field Theory, Phys. Lett. B 718 (2013) 1119-1124, [arXiv:1202.1301].

[280] H. Wei, Z.-C. Chen, and J. Liu, Cosmological Constraints on Variable Warm Dark Matter, arXiv:1302.0643.

[281] S. Bashinsky and U. Seljak, Neutrino perturbations in CMB anisotropy and matter clustering, Phys.Rev. D69 (2004) 083002, astro-ph/0310198.

[282] J. Silk, Cosmic black body radiation and galaxy formation, Astrophys.J. 151 (1968) 459-471.

[283] W. Hu and N. Sugiyama, Anisotropies in the cosmic microwave background: An Analytic approach, Astrophys.J. 444 (1995) 489-506, astro-ph/9407093.

[284] W. Hu, D. Scott, N. Sugiyama, and . White, Martin J., The Effect of physical assumptions on the calculation of microwave background anisotropies, Phys.Rev. D52 (1995) 5498-5515, astro-ph/9505043.

[285] W. Hu and N. Sugiyama, Small scale cosmological perturbations: An Analytic approach, Astrophys.J. 471 (1996) 542-570, astro-ph/9510117].

[286] W. Hu, D. J. Eisenstein, M. Tegmark, and M. J. White, Observationally determining the properties of dark matter, Phys.Rev. D59 (1999) 023512, astro-ph/9806362.

[287] R. Bowen, S. H. Hansen, A. Melchiorri, J. Silk, and R. Trotta, The Impact of an extra background of relativistic particles on the cosmological parameters derived from microwave background anisotropies, Mon.Not.Roy.Astron.Soc. 334 (2002) 760, astro-ph/0110636.

[288] Z. Hou, R. Keisler, L. Knox, M. Millea, and C. Reichardt, How Massless Neutrinos Affect the Cosmic Microwave Background Damping Tail, arXiv:1104.2333.

[289] R. Keisler, C. Reichardt, K. Aird, B. Benson, L. Bleem, et al., A Measurement of the Damping Tail of the Cosmic Microwave Background Power Spectrum with the South Pole Telescope, Astrophys.J. 743 (2011) 28, arXiv:1105.3182]. 
[290] J. Dunkley, R. Hlozek, J. Sievers, V. Acquaviva, P. Ade, et al., The Atacama Cosmology Telescope: Cosmological Parameters from the 2008 Power Spectra, Astrophys.J. 739 (2011) 52, arXiv:1009.0866.

[291] Planck Collaboration Collaboration, P. Ade et al., Planck 2013 results. I. Overview of products and scientific results, arXiv:1303.5062.

[292] Z. Hou, C. Reichardt, K. Story, B. Follin, R. Keisler, et al., Constraints on Cosmology from the Cosmic Microwave Background Power Spectrum of the 2500-square degree SPT-SZ Survey, arXiv:1212.6267.

[293] J. Dunkley, E. Calabrese, J. Sievers, G. Addison, N. Battaglia, et al., The Atacama Cosmology Telescope: likelihood for small-scale CMB data, arXiv:1301.0776.

[294] J. L. Sievers, R. A. Hlozek, M. R. Nolta, V. Acquaviva, G. E. Addison, et al., The Atacama Cosmology Telescope: Cosmological parameters from three seasons of data, arXiv:1301.0824.

[295] E. Di Valentino, S. Galli, M. Lattanzi, A. Melchiorri, P. Natoli, et al., Tickling the CMB damping tail: scrutinizing the tension between the ACT and SPT experiments, arXiv:1301.7343.

[296] E. Calabrese, R. A. Hlozek, N. Battaglia, E. S. Battistelli, J. R. Bond, et al., Cosmological Parameters from Pre-Planck CMB Measurements, arXiv:1302.1841.

[297] R. Hlozek, J. Dunkley, G. Addison, J. W. Appel, J. R. Bond, et al., The Atacama Cosmology Telescope: a measurement of the primordial power spectrum, Astrophys.J. $\mathbf{7 4 9}$ (2012) 90, arXiv:1105.4887].

[298] F. Bernardeau, S. Colombi, E. Gaztanaga, and R. Scoccimarro, Large scale structure of the universe and cosmological perturbation theory, Phys.Rept. 367 (2002) 1-248, astro-ph/0112551.

[299] Y. Zeldovich, Gravitational instability: An Approximate theory for large density perturbations, Astron.Astrophys. 5 (1970) 84-89.

[300] A. Lewis, A. Challinor, and A. Lasenby, Efficient computation of CMB anisotropies in closed FRW models, Astrophys. J. 538 (2000) 473-476, astro-ph/9911177.

[301] A. Lewis and S. Bridle, Cosmological parameters from CMB and other data: a MonteCarlo approach, Phys. Rev. D66 (2002) 103511, astro-ph/0205436.

[302] D. Blas, J. Lesgourgues, and T. Tram, The Cosmic Linear Anisotropy Solving System (CLASS) II: Approximation schemes, JCAP 1107 (2011) 034, arXiv:1104.2933.

[303] W. H. Press and P. Schechter, Formation of galaxies and clusters of galaxies by selfsimilar gravitational condensation, Astrophys.J. 187 (1974) 425-438.

[304] J. Peacock and A. Heavens, Alternatives to the Press-Schechter cosmological mass function, Mon.Not.Roy.Astron.Soc. 243 (1990) 133-143. 
[305] J. Bond, S. Cole, G. Efstathiou, and N. Kaiser, Excursion set mass functions for hierarchical Gaussian fluctuations, Astrophys.J. 379 (1991) 440.

[306] J. S. Bullock, T. S. Kolatt, Y. Sigad, R. S. Somerville, A. V. Kravtsov, et al., Profiles of dark haloes. Evolution, scatter, and environment, Mon.Not.Roy.Astron.Soc. 321 (2001) 559-575, astro-ph/9908159.

[307] V. Springel, S. D. White, A. Jenkins, C. S. Frenk, N. Yoshida, et al., Simulating the joint evolution of quasars, galaxies and their large-scale distribution, Nature 435 (2005) 629-636, astro-ph/0504097.

[308] V. Springel, The Cosmological simulation code GADGET-2, Mon.Not.Roy.Astron.Soc. 364 (2005) 1105-1134, astro-ph/0505010.

[309] M. Boylan-Kolchin, V. Springel, S. D. White, A. Jenkins, and G. Lemson, Resolving Cosmic Structure Formation with the Millennium-II Simulation, Mon.Not.Roy.Astron.Soc. 398 (2009) 1150, [arXiv:0903.3041].

[310] F. Prada, A. A. Klypin, A. J. Cuesta, J. E. Betancort-Rijo, and J. Primack, Halo concentrations in the standard LCDM cosmology, Mon.Not.Roy.Astron.Soc. 423 (2011) 3018-3030.

[311] A. A. Klypin, S. Trujillo-Gomez, and J. Primack, Dark Matter Halos in the Standard Cosmological Model: Results from the Bolshoi Simulation, The Astrophysical Journal $\mathbf{7 4 0}$ (2011).

[312] M. R. Lovell, V. Eke, C. S. Frenk, L. Gao, A. Jenkins, et al., The Haloes of Bright Satellite Galaxies in a Warm Dark Matter Universe, Mon.Not.Roy.Astron.Soc. 420 (2012) 2318-2324, [arXiv:1104.2929].

[313] S. Matarrese and M. Pietroni, Resumming Cosmic Perturbations, JCAP 0706 (2007) 026, astro-ph/0703563.

[314] H. de Vega, P. Salucci, and N. Sanchez, The mass of the dark matter particle from theory and observations, New Astron. 17 (2012) 653-666, arXiv:1004.1908.

[315] S. Tassev and M. Zaldarriaga, The Mildly Non-Linear Regime of Structure Formation, JCAP 1204 (2012) 013, arXiv:1109.4939.

[316] S. Anselmi and M. Pietroni, Nonlinear Power Spectrum from Resummed Perturbation Theory: a Leap Beyond the BAO Scale, JCAP 1212 (2012) 013, arXiv:1205.2235.

[317] C. Rampf and T. Buchert, Lagrangian perturbations and the matter bispectrum I: fourth-order model for non-linear clustering, JCAP 1206 (2012) 021, arXiv:1203.4260.

[318] C. Rampf and G. Rigopoulos, Zel'dovich Approximation and General Relativity, arXiv:1210.5446.

[319] S. Tassev and M. Zaldarriaga, Estimating CDM Particle Trajectories in the Mildly Non-Linear Regime of Structure Formation. Implications for the Density Field in Real and Redshift Space, JCAP 1212 (2012) 011, [arXiv:1203.5785]. 
[320] M. Maggiore and A. Riotto, The Halo Mass Function from Excursion Set Theory. I. Gaussian fluctuations with non-Markovian dependence on the smoothing scale, Astrophys.J. 711 (2010) 907-927, [arXiv:0903.1249].

[321] M. Maggiore and A. Riotto, The Halo mass function from excursion set theory. II. The diffusing barrier, Astrophys.J. 717 (2010) 515-525, [arXiv:0903.1250].

[322] M. Maggiore and A. Riotto, The Halo mass function from excursion set theory. III. Non-Gaussian fluctuations, Astrophys.J. 717 (2010) 526-541, arXiv:0903.1251.

[323] SDSS Collaboration Collaboration, W. J. Percival et al., Baryon Acoustic Oscillations in the Sloan Digital Sky Survey Data Release 7 Galaxy Sample, Mon.Not.Roy.Astron.Soc. 401 (2010) 2148-2168, arXiv:0907.1660.

[324] E. Papastergis, A. M. Martin, R. Giovanelli, and M. P. Haynes, The velocity width function of galaxies from the 40light on the cold dark matter overabundance problem, Astrophys.J. 739 (2011) 38, arXiv:1106.0710.

[325] F. Beutler, C. Blake, M. Colless, D. H. Jones, L. Staveley-Smith, et al., The 6dF Galaxy Survey: Baryon Acoustic Oscillations and the Local Hubble Constant, Mon.Not.Roy.Astron.Soc. 416 (2011) 3017-3032, [arXiv:1106.3366].

[326] C. Blake, E. Kazin, F. Beutler, T. Davis, D. Parkinson, et al., The WiggleZ Dark Energy Survey: mapping the distance-redshift relation with baryon acoustic oscillations, Mon.Not.Roy.Astron.Soc. 418 (2011) 1707-1724, [arXiv:1108.2635].

[327] SDSS Collaboration Collaboration, H. Aihara et al., The Eighth Data Release of the Sloan Digital Sky Survey: First Data from SDSS-III, Astrophys.J.Suppl. 193 (2011) 29, [arXiv:1101.1559].

[328] L. Anderson, E. Aubourg, S. Bailey, D. Bizyaev, M. Blanton, et al., The clustering of galaxies in the SDSS-III Baryon Oscillation Spectroscopic Survey: Baryon Acoustic Oscillations in the Data Release 9 Spectroscopic Galaxy Sample, Mon.Not.Roy.Astron.Soc. 428 (2013) 1036-1054, arXiv:1203.6594].

[329] C. Blake, S. Brough, M. Colless, C. Contreras, W. Couch, et al., The WiggleZ Dark Energy Survey: Joint measurements of the expansion and growth history at $z<1$, Mon.Not.Roy.Astron.Soc. 425 (2012) 405-414, arXiv:1204.3674].

[330] N. Padmanabhan, X. Xu, D. J. Eisenstein, R. Scalzo, A. J. Cuesta, et al., A 2Methods and Application to the Sloan Digital Sky Survey, arXiv:1202.0090.

[331] G. B. Poole, C. Blake, D. Parkinson, S. Brough, M. Colless, et al., The WiggleZ Dark Energy Survey: Probing the Epoch of Radiation Domination using Large Scale Structure, arXiv:1211.5605.

[332] A. Dolgov and F. Villante, BBN bounds on active sterile neutrino mixing, Nucl.Phys. B679 (2004) 261-298, hep-ph/0308083. 
[333] M. Cirelli, G. Marandella, A. Strumia, and F. Vissani, Probing oscillations into sterile neutrinos with cosmology, astrophysics and experiments, Nucl.Phys. B708 (2005) 215-267, hep-ph/0403158.

[334] A. Mirizzi, N. Saviano, G. Miele, and P. D. Serpico, Light sterile neutrino production in the early universe with dynamical neutrino asymmetries, Phys.Rev. D86 (2012) 053009, [arXiv:1206.1046].

[335] A. G. Riess, L. Macri, S. Casertano, H. Lampeitl, H. C. Ferguson, et al., A 3Telescope and Wide Field Camera 3, Astrophys.J. 730 (2011) 119, arXiv:1103.2976.

[336] M. Gonzalez-Garcia, M. Maltoni, and J. Salvado, Robust Cosmological Bounds on Neutrinos and their Combination with Oscillation Results, JHEP 1008 (2010) 117, [arXiv:1006.3795].

[337] L. M. Krauss, C. Lunardini, and C. Smith, Neutrinos, WMAP, and BBN, Phys.Rev.D (2010) arXiv:1009.4666.

[338] M. Archidiacono, N. Fornengo, C. Giunti, and A. Melchiorri, Testing 3+1 and 3+2 neutrino mass models with cosmology and short baseline experiments, Phys.Rev. D86 (2012) 065028, [arXiv:1207.6515].

[339] E. Giusarma, R. de Putter, and O. Mena, Testing standard and non-standard neutrino physics with cosmological data, arXiv:1211.2154

[340] S. Riemer-Sorensen, D. Parkinson, T. M. Davis, and C. Blake, Simultaneous constraints on the number and mass of relativistic species, Astrophys.J. $\mathbf{7 6 3}$ (2013) 89, [arXiv:1210.2131].

[341] S. Joudaki, K. N. Abazajian, and M. Kaplinghat, Are Light Sterile Neutrinos Preferred or Disfavored by Cosmology?, arXiv:1208.4354.

[342] S. M. Feeney, H. V. Peiris, and L. Verde, Is there evidence for additional neutrino species from cosmology?, arXiv:1302.0014.

[343] S. Riemer-Sorensen, D. Parkinson, and T. M. Davis, What is half a neutrino? Reviewing cosmological constraints on neutrinos and dark radiation, arXiv:1301.7102.

[344] M. Archidiacono, E. Giusarma, S. Hannestad, and O. Mena, Cosmic dark radiation and neutrinos, arXiv:1307.0637.

[345] M. Wyman, D. H. Rudd, R. A. Vanderveld, and W. Hu, nu-LCDM: Neutrinos reconcile Planck with the Local Universe, arXiv:1307.7715.

[346] J. Hamann and J. Hasenkamp, A new life for sterile neutrinos: resolving inconsistencies using hot dark matter, arXiv:1308.3255.

[347] R. A. Battye and A. Moss, Evidence for massive neutrinos from CMB and lensing observations, arXiv:1308.5870.

[348] S. Gariazzo, C. Giunti, and M. Laveder, Light Sterile Neutrinos in Cosmology and Short-Baseline Oscillation Experiments, arXiv:1309.3192. 
[349] A. Mirizzi, G. Mangano, N. Saviano, E. Borriello, C. Giunti, et al., The strongest bounds on active-sterile neutrino mixing after Planck data, arXiv:1303.5368.

[350] L. Verde, R. Jimenez, and S. Feeney, The importance of local measurements for cosmology, arXiv:1303.5341.

[351] Planck Collaboration Collaboration, The Scientific programme of planck, astro-ph/0604069.

[352] R. Foot and R. Volkas, Reconciling sterile neutrinos with big bang nucleosynthesis, Phys.Rev.Lett. 75 (1995) 4350, hep-ph/9508275].

[353] C. M. Ho and R. J. Scherrer, Sterile Neutrinos and Light Dark Matter Save Each Other, arXiv:1212.1689.

[354] D. J. Schwarz and M. Stuke, Does the CMB prefer a leptonic Universe?, arXiv:1211.6721.

[355] X.-D. Shi, Chaotic amplification of neutrino chemical potentials by neutrino oscillations in big bang nucleosynthesis, Phys.Rev. D54 (1996) 2753-2760, astro-ph/9602135.

[356] N. F. Bell, R. R. Volkas, and Y. Y. Wong, Relic neutrino asymmetry evolution from first principles, Phys.Rev. D59 (1999) 113001, hep-ph/9809363.

[357] S. Hannestad and G. Raffelt, Imprint of sterile neutrinos in the cosmic microwave background radiation, Phys.Rev. D59 (1999) 043001, astro-ph/9805223.

[358] K. Abazajian, G. M. Fuller, and M. Patel, Sterile neutrino hot, warm, and cold dark matter, Phys. Rev. D64 (2001) 023501, astro-ph/0101524.

[359] K. Abazajian, N. F. Bell, G. M. Fuller, and Y. Y. Wong, Cosmological lepton asymmetry, primordial nucleosynthesis, and sterile neutrinos, Phys.Rev. D72 (2005) 063004, astro-ph/0410175.

[360] Y.-Z. Chu and M. Cirelli, Sterile neutrinos, lepton asymmetries, primordial elements: How much of each?, Phys.Rev. D74 (2006) 085015, astro-ph/0608206.

[361] A. Melchiorri, O. Mena, S. Palomares-Ruiz, S. Pascoli, A. Slosar, et al., Sterile Neutrinos in Light of Recent Cosmological and Oscillation Data: A Multi-Flavor Scheme Approach, JCAP 0901 (2009) 036, arXiv:0810.5133].

[362] D. Kirilova, BBN with Late Electron-Sterile Neutrino Oscillations: The Finest Leptometer, JCAP 1206 (2012) 007, arXiv:1101.4177.

[363] S. Hannestad, I. Tamborra, and T. Tram, Thermalisation of light sterile neutrinos in the early universe, JCAP 1207 (2012) 025, [arXiv:1204.5861].

[364] T. D. Jacques, L. M. Krauss, and C. Lunardini, Additional Light Sterile Neutrinos and Cosmology, arXiv:1301.3119. 
[365] N. Saviano, A. Mirizzi, O. Pisanti, P. D. Serpico, G. Mangano, et al., Multi-momentum and multi-flavour active-sterile neutrino oscillations in the early universe: role of neutrino asymmetries and effects on nucleosynthesis, arXiv:1302.1200,

[366] S. Hannestad, R. S. Hansen, and T. Tram, Can active-sterile neutrino oscillations lead to chaotic behavior of the cosmological lepton asymmetry?, arXiv:1302.7279.

[367] J. Hamann, Evidence for extra radiation? Profile likelihood versus Bayesian posterior, JCAP 1203 (2012) 021, arXiv:1110.4271.

[368] B. Audren, J. Lesgourgues, K. Benabed, and S. Prunet, Conservative Constraints on Early Cosmology: an illustration of the Monte Python cosmological parameter inference code, arXiv:1210.7183

[369] M.-R. Wu, T. Fischer, G. Martnez-Pinedo, and Y.-Z. Qian, Are Light Sterile Neutrinos Consistent with Supernova Explosions?, arXiv:1305.2382.

[370] J. Hidaka and G. M. Fuller, Dark matter sterile neutrinos in stellar collapse: Alteration of energy/lepton number transport and a mechanism for supernova explosion enhancement, Phys.Rev. D74 (2006) 125015, astro-ph/0609425.

[371] J. Hidaka and G. M. Fuller, Sterile Neutrino-Enhanced Supernova Explosions, Phys.Rev. D76 (2007) 083516, arXiv:0706.3886.

[372] G. M. Fuller, A. Kusenko, and K. Petraki, Heavy sterile neutrinos and supernova explosions, Phys.Lett. B670 (2009) 281-284, arXiv:0806.4273.

[373] A. Kusenko and G. Segre, Pulsar kicks from neutrino oscillations, Phys.Rev. D59 (1999) 061302, astro-ph/9811144.

[374] G. M. Fuller, A. Kusenko, I. Mocioiu, and S. Pascoli, Pulsar kicks from a dark-matter sterile neutrino, Phys.Rev. D68 (2003) 103002, astro-ph/0307267.

[375] A. D. Sakharov, Violation of CP Invariance, c Asymmetry, and Baryon Asymmetry of the Universe, Pisma Zh. Eksp. Teor. Fiz. 5 (1967) 32-35.

[376] M. Kobayashi and T. Maskawa, CP Violation in the Renormalizable Theory of Weak Interaction, Prog.Theor.Phys. 49 (1973) 652-657.

[377] C. Wu, E. Ambler, R. Hayward, D. Hoppes, and R. Hudson, EXPERIMENTAL TEST OF PARITY CONSERVATION IN BETA DECAY, Phys.Rev. 105 (1957) 1413-1414.

[378] J. Christenson, J. Cronin, V. Fitch, and R. Turlay, Evidence for the 2 pi Decay of the k(2)0 Meson, Phys.Rev.Lett. 13 (1964) 138-140.

[379] M. Fukugita and T. Yanagida, Baryogenesis Without Grand Unification, Phys. Lett. B174 (1986) 45.

[380] H. A. Weldon, Effective Fermion Masses of Order gT in High Temperature Gauge Theories with Exact Chiral Invariance, Phys.Rev. D26 (1982) 2789. 
[381] H. A. Weldon, Covariant Calculations at Finite Temperature: The Relativistic Plasma, Phys.Rev. D26 (1982) 1394.

[382] K. Dick, M. Lindner, M. Ratz, and D. Wright, Leptogenesis with Dirac neutrinos, Phys.Rev.Lett. 84 (2000) 4039-4042, hep-ph/9907562.

[383] S. Y. Khlebnikov and M. Shaposhnikov, The Statistical Theory of Anomalous Fermion Number Nonconservation, Nucl.Phys. B308 (1988) 885-912.

[384] M. Laine and M. E. Shaposhnikov, A Remark on sphaleron erasure of baryon asymmetry, Phys.Rev. D61 (2000) 117302, hep-ph/9911473.

[385] W. Buchmuller and M. Plumacher, Spectator processes and baryogenesis, Phys.Lett. B511 (2001) 74-76, hep-ph/0104189.

[386] E. Nardi, Y. Nir, J. Racker, and E. Roulet, On Higgs and sphaleron effects during the leptogenesis era, JHEP 0601 (2006) 068, hep-ph/0512052.

[387] W. Buchmuller, R. Peccei, and T. Yanagida, Leptogenesis as the origin of matter, Ann.Rev.Nucl.Part.Sci. 55 (2005) 311-355, hep-ph/0502169.

[388] S. Blanchet and P. Di Bari, The minimal scenario of leptogenesis, New J.Phys. 14 (2012) 125012, arXiv:1211.0512].

[389] C. S. Fong, E. Nardi, and A. Riotto, Leptogenesis in the Universe, Adv.High Energy Phys. 2012 (2012) 158303, arXiv:1301.3062.

[390] W. Buchmuller, P. Di Bari, and M. Plumacher, Leptogenesis for pedestrians, Annals Phys. 315 (2005) 305-351, hep-ph/0401240.

[391] S. Davidson and A. Ibarra, A Lower bound on the right-handed neutrino mass from leptogenesis, Phys.Lett. B535 (2002) 25-32, hep-ph/0202239.

[392] W. Buchmuller, P. Di Bari, and M. Plumacher, A Bound on neutrino masses from baryogenesis, Phys.Lett. B547 (2002) 128-132, hep-ph/0209301.

[393] W. Buchmuller, P. Di Bari, and M. Plumacher, The Neutrino mass window for baryogenesis, Nucl.Phys. B665 (2003) 445-468, hep-ph/0302092.

[394] E. Nardi, Leptogenesis and neutrino masses, Nucl.Phys.Proc.Suppl. 217 (2011) 27-32.

[395] R. Barbieri, P. Creminelli, A. Strumia, and N. Tetradis, Baryogenesis through leptogenesis, Nucl.Phys. B575 (2000) 61-77, hep-ph/9911315.

[396] S. Blanchet and P. Di Bari, Flavor effects on leptogenesis predictions, JCAP 0703 (2007) 018, hep-ph/0607330.

[397] A. Abada, S. Davidson, A. Ibarra, F.-X. Josse-Michaux, M. Losada, et al., Flavour Matters in Leptogenesis, JHEP 0609 (2006) 010, hep-ph/0605281.

[398] A. Abada, S. Davidson, F.-X. Josse-Michaux, M. Losada, and A. Riotto, Flavor issues in leptogenesis, JCAP 0604 (2006) 004, hep-ph/0601083. 
[399] E. Nardi, Y. Nir, E. Roulet, and J. Racker, The Importance of flavor in leptogenesis, JHEP 0601 (2006) 164, hep-ph/0601084.

[400] T. Endoh, T. Morozumi, and Z.-h. Xiong, Primordial lepton family asymmetries in seesaw model, Prog.Theor.Phys. 111 (2004) 123-149, hep-ph/0308276.

[401] S. Antusch, S. Blanchet, M. Blennow, and E. Fernandez-Martinez, Non-unitary Leptonic Mixing and Leptogenesis, JHEP 1001 (2010) 017, [arXiv:0910.5957].

[402] J. Racker, M. Pena, and N. Rius, Leptogenesis with small violation of B-L, JCAP 1207 (2012) 030, arXiv:1205.1948.

[403] P. Di Bari, Seesaw geometry and leptogenesis, Nucl.Phys. B727 (2005) 318-354, hep-ph/0502082.

[404] G. Engelhard, Y. Grossman, E. Nardi, and Y. Nir, The Importance of N2 leptogenesis, Phys.Rev.Lett. 99 (2007) 081802, hep-ph/0612187.

[405] O. Vives, Flavor dependence of CP asymmetries and thermal leptogenesis with strong right-handed neutrino mass hierarchy, Phys.Rev. D73 (2006) 073006, hep-ph/0512160.

[406] S. Blanchet and P. Di Bari, New aspects of leptogenesis bounds, Nucl.Phys. B807 (2009) 155-187, arXiv:0807.0743.

[407] E. Bertuzzo, P. Di Bari, and L. Marzola, The problem of the initial conditions in flavoured leptogenesis and the tauon $N_{2}$-dominated scenario, Nucl.Phys. B849 (2011) 521-548, [arXiv:1007.1641].

[408] S. Antusch, P. Di Bari, D. A. Jones, and S. F. King, A fuller flavour treatment of $\mathrm{N}_{2}$-dominated leptogenesis, Nucl.Phys. B856 (2012) 180-209, [arXiv:1003.5132].

[409] S. Blanchet, P. Di Bari, D. A. Jones, and L. Marzola, Leptogenesis with heavy neutrino flavours: from density matrix to Boltzmann equations, JCAP 1301 (2013) 041, arXiv:1112.4528.

[410] A. Pilaftsis and T. E. Underwood, Resonant leptogenesis, Nucl.Phys. B692 (2004) 303-345, hep-ph/0309342.

[411] M. Garny, A. Kartavtsev, and A. Hohenegger, Leptogenesis from first principles in the resonant regime, arXiv: 1112.6428 .

[412] B. Garbrecht and M. Herranen, Effective Theory of Resonant Leptogenesis in the Closed-Time-Path Approach, Nucl.Phys. B861 (2012) 17-52, arXiv:1112.5954.

[413] J. Liu and G. Segre, Unstable particle mixing and CP violation in weak decays, Phys.Rev. D49 (1994) 1342-1349, hep-ph/9310248.

[414] M. Flanz, E. A. Paschos, U. Sarkar, and J. Weiss, Baryogenesis through mixing of heavy Majorana neutrinos, Phys.Lett. B389 (1996) 693-699, hep-ph/9607310.

[415] L. Covi and E. Roulet, Baryogenesis from mixed particle decays, Phys.Lett. B399 (1997) 113-118, hep-ph/9611425. 
[416] A. Pilaftsis, Resonant CP violation induced by particle mixing in transition amplitudes, Nucl.Phys. B504 (1997) 61-107, hep-ph/9702393.

[417] W. Buchmuller and M. Plumacher, CP asymmetry in Majorana neutrino decays, Phys.Lett. B431 (1998) 354-362, hep-ph/9710460.

[418] C. S. Fong, M. Gonzalez-Garcia, E. Nardi, and J. Racker, Supersymmetric Leptogenesis, JCAP 1012 (2010) 013, arXiv:1009.0003.

[419] B. Garbrecht and M. J. Ramsey-Musolf, Cuts, Cancellations and the Closed Time Path: The Soft Leptogenesis Example, arXiv:1307.0524.

[420] B. Garbrecht, Leptogenesis from Additional Higgs Doublets, Phys.Rev. D85 (2012) 123509, [arXiv:1201.5126].

[421] C. S. Fong, M. Gonzalez-Garcia, E. Nardi, and E. Peinado, New ways to TeV scale leptogenesis, arXiv:1305.6312.

[422] M. Drewes and B. Garbrecht, Leptogenesis from a GeV Seesaw without Mass Degeneracy, JHEP 1303 (2013) 096, arXiv:1206.5537.

[423] E. K. Akhmedov, V. A. Rubakov, and A. Y. Smirnov, Baryogenesis via neutrino oscillations, Phys. Rev. Lett. 81 (1998) 1359-1362, hep-ph/9803255.

[424] L. Canetti and M. Shaposhnikov, Baryon Asymmetry of the Universe in the NuMSM, JCAP 1009 (2010) 001, arXiv:1006.0133].

[425] V. V. Khoze and G. Ro, Leptogenesis and Neutrino Oscillations in the Classically Conformal Standard Model with the Higgs Portal, arXiv:1307.3764.

[426] D. E. Morrissey and M. J. Ramsey-Musolf, Electroweak baryogenesis, New J.Phys. 14 (2012) 125003, arXiv:1206.2942.

[427] S. Blanchet, Z. Chacko, and R. N. Mohapatra, Neutrino Mass Seesaw at the Weak Scale, the Baryon Asymmetry, and the LHC, Phys.Rev. D80 (2009) 085002, arXiv:0812.3837.

[428] S. Hollenberg, H. Pas, and D. Schalla, Baryon asymmetry of the universe and new neutrino states, arXiv:1110.0948.

[429] G. Sigl and G. Raffelt, General kinetic description of relativistic mixed neutrinos, Nucl. Phys. B406 (1993) 423-451.

[430] M. Beneke, B. Garbrecht, C. Fidler, M. Herranen, and P. Schwaller, Flavoured Leptogenesis in the CTP Formalism, Nucl.Phys. B843 (2011) 177-212, arXiv: 1007.4783].

[431] F. Hahn-Woernle, M. Plumacher, and Y. Wong, Full Boltzmann equations for leptogenesis including scattering, JCAP 0908 (2009) 028, arXiv:0907.0205.

[432] T. Asaka, S. Eijima, and H. Ishida, Kinetic Equations for Baryogenesis via Sterile Neutrino Oscillation, JCAP 1202 (2012) 021, [arXiv:1112.5565]. 
[433] E. W. Kolb and S. Wolfram, Baryon Number Generation in the Early Universe, Nucl.Phys. B172 (1980) 224.

[434] Y. Grossman, T. Kashti, Y. Nir, and E. Roulet, Leptogenesis from supersymmetry breaking, Phys.Rev.Lett. 91 (2003) 251801, hep-ph/0307081.

[435] G. D'Ambrosio, G. F. Giudice, and M. Raidal, Soft leptogenesis, Phys.Lett. B575 (2003) 75-84, hep-ph/0308031.

[436] Y. Grossman, T. Kashti, Y. Nir, and E. Roulet, New ways to soft leptogenesis, JHEP 0411 (2004) 080, hep-ph/0407063.

[437] C. S. Fong and M. Gonzalez-Garcia, On Gaugino Contributions to Soft Leptogenesis, JHEP 0903 (2009) 073, [arXiv:0901.0008].

[438] T. Frossard, M. Garny, A. Hohenegger, A. Kartavtsev, and D. Mitrouskas, Systematic approach to thermal leptogenesis, Phys.Rev. D87 (2013) 085009, arXiv:1211.2140].

[439] T. Prokopec, M. G. Schmidt, and S. Weinstock, Transport equations for chiral fermions to order $h$ bar and electroweak baryogenesis. Part 1, Annals Phys. 314 (2004) 208-265, hep-ph/0312110.

[440] T. Prokopec, M. G. Schmidt, and S. Weinstock, Transport equations for chiral fermions to order h-bar and electroweak baryogenesis. Part II, Annals Phys. 314 (2004) 267-320, hep-ph/0406140.

[441] A. Anisimov, W. Buchmuller, M. Drewes, and S. Mendizabal, Nonequilibrium Dynamics of Scalar Fields in a Thermal Bath, Annals Phys. 324 (2009) 1234-1260, [arXiv:0812.1934].

[442] M. Drewes, On the Role of Quasiparticles and thermal Masses in Nonequilibrium Processes in a Plasma, arXiv:1012.5380.

[443] P. Millington and A. Pilaftsis, Perturbative Non-Equilibrium Thermal Field Theory, arXiv:1211.3152.

[444] W. Buchmuller and S. Fredenhagen, Quantum mechanics of baryogenesis, Phys.Lett. B483 (2000) 217-224, hep-ph/0004145.

[445] A. De Simone and A. Riotto, Quantum Boltzmann Equations and Leptogenesis, JCAP 0708 (2007) 002, hep-ph/0703175.

[446] M. Garny, A. Hohenegger, A. Kartavtsev, and M. Lindner, Systematic approach to leptogenesis in nonequilibrium QFT: Vertex contribution to the CP-violating parameter, Phys.Rev. D80 (2009) 125027, arXiv:0909.1559].

[447] V. Cirigliano, C. Lee, M. J. Ramsey-Musolf, and S. Tulin, Flavored Quantum Boltzmann Equations, Phys.Rev. D81 (2010) 103503, arXiv:0912.3523].

[448] M. Garny, A. Hohenegger, A. Kartavtsev, and M. Lindner, Systematic approach to leptogenesis in nonequilibrium QFT: Self-energy contribution to the CP-violating parameter, Phys.Rev. D81 (2010) 085027, arXiv:0911.4122. 
[449] A. Anisimov, W. Buchmuller, M. Drewes, and S. Mendizabal, Leptogenesis from Quantum Interference in a Thermal Bath, Phys.Rev.Lett. 104 (2010) 121102, [arXiv:1001.3856.

[450] J.-S. Gagnon and M. Shaposhnikov, Baryon Asymmetry of the Universe without Boltzmann or Kadanoff-Baym equations, Phys.Rev. D83 (2011) 065021, arXiv:1012.1126.

[451] M. Beneke, B. Garbrecht, M. Herranen, and P. Schwaller, Finite Number Density Corrections to Leptogenesis, Nucl.Phys. B838 (2010) 1-27, [arXiv:1002.1326].

[452] A. Anisimov, W. Buchmuller, M. Drewes, and S. Mendizabal, Quantum Leptogenesis I, Annals Phys. 326 (2011) 1998-2038, [arXiv:1012.5821].

[453] M. Garny, A. Hohenegger, and A. Kartavtsev, Quantum corrections to leptogenesis from the gradient expansion, arXiv:1005.5385.

[454] M. Herranen, K. Kainulainen, and P. M. Rahkila, Coherent quantum Boltzmann equations from cQPA, JHEP 1012 (2010) 072, [arXiv:1006.1929].

[455] M. Herranen, K. Kainulainen, and P. M. Rahkila, Flavour-coherent propagators and Feynman rules: Covariant cQPA formulation, JHEP 1202 (2012) 080, [arXiv:1108.2371].

[456] C. Fidler, M. Herranen, K. Kainulainen, and P. M. Rahkila, Flavoured quantum Boltzmann equations from cQPA, JHEP 1202 (2012) 065, arXiv:1108.2309.

[457] B. Garbrecht, Baryogenesis from Mixing of Lepton Doublets, Nucl.Phys. B868 (2013) 557-576, [arXiv:1210.0553].

[458] T. Frossard, A. Kartavtsev, and D. Mitrouskas, Systematic approach to Delta L=1 processes in thermal leptogenesis, arXiv:1304.1719.

[459] J. v. Neumann, Wahrscheinlichkeitstheoretischer Aufbau der Quantenmechanik, Nachr. Ges. Wiss. Gottingen Mathematisch-Physikalische Klasse (1927) 245-272.

[460] J. v. Neumann, Thermodynamik quantenmechanischer Gesamtheiten, Nachr. Ges. Wiss. Gottingen Mathematisch-Physikalische Klasse (1927) 273-291.

[461] L. P. Kadanoff and G. Baym, Quantum Statistical Mechanics, Benjamin, New York (1962).

[462] J. S. Schwinger, Brownian motion of a quantum oscillator, J.Math.Phys. 2 (1961) 407-432.

[463] P. M. Bakshi and K. T. Mahanthappa, Expectation value formalism in quantum field theory. 1., J.Math.Phys. 4 (1963) 1-11.

[464] P. M. Bakshi and K. T. Mahanthappa, Expectation value formalism in quantum field theory. 2., J.Math.Phys. 4 (1963) 12-16.

[465] L. Keldysh, Diagram technique for nonequilibrium processes, Zh.Eksp.Teor.Fiz. 47 (1964) 1515-1527. 
[466] Y. Burnier, M. Laine, and M. Shaposhnikov, Baryon and lepton number violation rates across the electroweak crossover, JCAP 0602 (2006) 007, hep-ph/0511246.

[467] M. D'Onofrio, K. Rummukainen, and A. Tranberg, The sphaleron rate at the electroweak crossover with 125 GeV Higgs mass, PoS LATTICE2012 (2012) 055, [arXiv:1212.3206].

[468] T. Asaka, M. Laine, and M. Shaposhnikov, On the hadronic contribution to sterile neutrino production, JHEP 06 (2006) 053, hep-ph/0605209.

[469] M. Laine and Y. Schroder, Thermal right-handed neutrino production rate in the non-relativistic regime, JHEP 1202 (2012) 068, arXiv:1112.1205.

[470] A. Salvio, P. Lodone, and A. Strumia, Towards leptogenesis at NLO: the right-handed neutrino interaction rate, JHEP 1108 (2011) 116, [arXiv:1106.2814].

[471] D. Besak and D. Bodeker, Thermal production of ultrarelativistic right-handed neutrinos: Complete leading-order results, JCAP 1203 (2012) 029, arXiv:1202.1288.

[472] B. Garbrecht, F. Glowna, and M. Herranen, Right-Handed Neutrino Production at Finite Temperature: Radiative Corrections, Soft and Collinear Divergences, arXiv:1302.0743.

[473] B. Garbrecht, F. Glowna, and P. Schwaller, Scattering Rates For Leptogenesis: Damping of Lepton Flavour Coherence and Production of Singlet Neutrinos, arXiv:1303.5498.

[474] M. Laine, Thermal right-handed neutrino production rate in the relativistic regime, JHEP 1308 (2013) 138, [arXiv:1307.4909].

[475] S. Biondini, N. Brambilla, M. A. Escobedo, and A. Vairo, An effective field theory for non-relativistic Majorana neutrinos, arXiv:1307.7680.

[476] C. P. Kiessig, M. Plumacher, and M. H. Thoma, Decay of a Yukawa fermion at finite temperature and applications to leptogenesis, Phys.Rev. D82 (2010) 036007, [arXiv:1003.3016].

[477] C. Kiessig and M. Plumacher, Hard-Thermal-Loop Corrections in Leptogenesis I: CP-Asymmetries, JCAP 1207 (2012) 014, arXiv:1111.1231.

[478] C. Kiessig and M. Plumacher, Hard-Thermal-Loop Corrections in Leptogenesis II: Solving the Boltzmann Equations, arXiv:1111.1235.

[479] K. Miura, Y. Hidaka, D. Satow, and T. Kunihiro, Neutrino spectral density at electroweak scale temperature, arXiv:1306.1701

[480] M. Laine, Thermal 2-loop master spectral function at finite momentum, arXiv:1304.0202.

[481] J. H. Oort, The force exerted by the stellar system in the direction perpendicular to the galactic plane and some related problems, Bulletin of the Astronomical Institutes of the Netherlands 6 (1932) 249.

[482] F. Zwicky, Die Rotverschiebung von extragalaktischen Nebeln, Helv.Phys.Acta 6 (1933) $110-127$. 
[483] G. R. Blumenthal, S. Faber, J. R. Primack, and M. J. Rees, Formation of Galaxies and Large Scale Structure with Cold Dark Matter, Nature 311 (1984) 517-525.

[484] M. Davis, G. Efstathiou, C. S. Frenk, and S. D. White, The Evolution of Large Scale Structure in a Universe Dominated by Cold Dark Matter, Astrophys.J. 292 (1985) 371-394.

[485] A. A. Klypin, A. V. Kravtsov, O. Valenzuela, and F. Prada, Where are the missing galactic satellites?, Astrophys. J. 522 (1999) 82-92, astro-ph/9901240.

[486] B. Moore, S. Ghigna, F. Governato, G. Lake, T. R. Quinn, et al., Dark matter substructure within galactic halos, Astrophys.J. 524 (1999) L19-L22, astro-ph/9907411.

[487] J. S. Bullock, A. V. Kravtsov, and D. H. Weinberg, Reionization and the abundance of galactic satellites, Astrophys.J. 539 (2000) 517, astro-ph/0002214.

[488] A. Benson, C. Frenk, C. G. Lacey, C. Baugh, and S. Cole, The effects of photoionization on galaxy formation. 2. Satellites in the local group, Mon.Not.Roy.Astron.Soc. 333 (2002) 177, astro-ph/0108218.

[489] R. S. Somerville, Can photoionization squelching resolve the sub-structure crisis?, Astrophys.J. 572 (2002) L23-L26, astro-ph/0107507].

[490] A. V. Maccio', X. Kang, F. Fontanot, R. S. Somerville, S. E. Koposov, et al., On the origin and properties of Ultrafaint Milky Way Satellites in a LCDM Universe, arXiv:0903.4681.

[491] M. Boylan-Kolchin, J. S. Bullock, and M. Kaplinghat, Too big to fail? The puzzling darkness of massive Milky Way subhaloes, Mon.Not.Roy.Astron.Soc. 415 (2011) L40, arXiv:1103.0007.

[492] J. R. Primack, Triumphs and tribulations of Lambda CDM, the double dark theory, Annalen Phys. 524 (2012) 535-544.

[493] K. A. Olive and M. S. Turner, COSMOLOGICAL BOUNDS ON THE MASSES OF STABLE, RIGHT-HANDED NEUTRINOS, Phys.Rev. D25 (1982) 213.

[494] A. D. Dolgov and S. H. Hansen, Massive sterile neutrinos as warm dark matter, Astropart. Phys. 16 (2002) 339-344, hep-ph/0009083.

[495] K. Abazajian, G. M. Fuller, and W. H. Tucker, Direct detection of warm dark matter in the X-ray, Astrophys.J. 562 (2001) 593-604, astro-ph/0106002.

[496] M. Viel and M. G. Haehnelt, Cosmological and astrophysical parameters from the SDSS flux power spectrum and hydrodynamical simulations of the Lyman-alpha forest, Mon.Not.Roy.Astron.Soc. 365 (2006) 231-244, astro-ph/0508177.

[497] A. Boyarsky, A. Neronov, O. Ruchayskiy, and M. Shaposhnikov, Constraints on sterile neutrino as a dark matter candidate from the diffuse $x$-ray background, Mon. Not. Roy. Astron. Soc. 370 (2006) 213-218, astro-ph/0512509.

[498] A. Boyarsky, A. Neronov, O. Ruchayskiy, M. Shaposhnikov, and I. Tkachev, Where to find a dark matter sterile neutrino?, Phys.Rev.Lett. 97 (2006) 261302, astro-ph/0603660. 
[499] A. Boyarsky, J. Nevalainen, and O. Ruchayskiy, Constraints on the parameters of radiatively decaying dark matter from the dark matter halo of the milky way and ursa minor, astro-ph/0610961.

[500] C. R. Watson, J. F. Beacom, H. Yuksel, and T. P. Walker, Direct X-ray Constraints on Sterile Neutrino Warm Dark Matter, Phys.Rev. D74 (2006) 033009, astro-ph/0605424.

[501] K. Abazajian and S. M. Koushiappas, Constraints on Sterile Neutrino Dark Matter, Phys.Rev. D74 (2006) 023527, astro-ph/0605271.

[502] K. N. Abazajian, M. Markevitch, S. M. Koushiappas, and R. C. Hickox, Limits on the radiative decay of sterile neutrino dark matter from the unresolved cosmic and soft x-ray backgrounds, astro-ph/0611144.

[503] S. Riemer-Sorensen, S. H. Hansen, and K. Pedersen, Sterile neutrinos in the Milky Way: Observational constraints, Astrophys.J. 644 (2006) L33-L36, astro-ph/0603661.

[504] M. Viel, J. Lesgourgues, M. G. Haehnelt, S. Matarrese, and A. Riotto, Can sterile neutrinos be ruled out as warm dark matter candidates?, Phys. Rev. Lett. 97 (2006) 071301, astro-ph/0605706.

[505] U. Seljak, A. Makarov, P. McDonald, and H. Trac, Can sterile neutrinos be the dark matter?, Phys. Rev. Lett. 97 (2006) 191303, astro-ph/0602430.

[506] P. Colin, O. Valenzuela, and V. Avila-Reese, On the Structure of Dark Matter Halos at the Damping Scale of the Power Spectrum with and without Relict Velocities, Astrophys.J. 673 (2008) 203-214, [arXiv:0709.4027].

[507] G. Belanger, A. Pukhov, and G. Servant, Dirac Neutrino Dark Matter, JCAP 0801 (2008) 009, arXiv:0706.0526.

[508] A. Boyarsky, D. Malyshev, A. Neronov, and O. Ruchayskiy, Constraining DM properties with SPI, Mon.Not.Roy.Astron.Soc. 387 (2008) 1345, [arXiv:0710.4922].

[509] A. Boyarsky, D. Iakubovskyi, O. Ruchayskiy, and V. Savchenko, Constraints on decaying Dark Matter from XMM-Newton observations of M31, Mon.Not.Roy.Astron.Soc. 387 (2008) 1361, [arXiv:0709.2301.

[510] A. Boyarsky, J. Lesgourgues, O. Ruchayskiy, and M. Viel, Lyman-alpha constraints on warm and on warm-plus-cold dark matter models, JCAP 0905 (2009) 012, arXiv:0812.0010].

[511] A. Boyarsky, J. Lesgourgues, O. Ruchayskiy, and M. Viel, Realistic sterile neutrino dark matter with keV mass does not contradict cosmological bounds, Phys. Rev. Lett. 102 (2009) 201304, [arXiv:0812.3256].

[512] A. Boyarsky, O. Ruchayskiy, and D. Iakubovskyi, A lower bound on the mass of Dark Matter particles, arXiv:0808.3902.

[513] M. Loewenstein, A. Kusenko, and P. L. Biermann, New Limits on Sterile Neutrinos from Suzaku Observations of the Ursa Minor Dwarf Spheroidal Galaxy, Astrophys.J. 700 (2009) 426-435, [arXiv:0812.2710]. 
[514] D. Gorbunov, A. Khmelnitsky, and V. Rubakov, Constraining sterile neutrino dark matter by phase-space density observations, JCAP 0810 (2008) 041, arXiv:0808.3910].

[515] A. Kusenko, Sterile neutrinos: The Dark side of the light fermions, Phys.Rept. 481 (2009) 1-28, arXiv:0906.2968.

[516] J. den Herder, A. Boyarsky, O. Ruchayskiy, K. Abazajian, C. Frenk, et al., The Search for decaying Dark Matter, arXiv:0906.1788.

[517] K. N. Abazajian, Detection of Dark Matter Decay in the X-ray, arXiv:0903.2040.

[518] J. Wu, C.-M. Ho, and D. Boyanovsky, Sterile neutrinos produced near the EW scale. I. Mixing angles, MSW resonances and production rates, Phys.Rev. D80 (2009) 103511, [arXiv:0902.4278].

[519] D. Boyanovsky and J. Wu, Small scale aspects of warm dark matter : power spectra and acoustic oscillations, Phys.Rev. D83 (2011) 043524, arXiv:1008.0992.

[520] D. Boyanovsky, Warm dark matter at small scales: peculiar velocities and phase space density, Phys.Rev. D83 (2011) 103504, [arXiv:1011.2217].

[521] R. M. Dunstan, K. N. Abazajian, E. Polisensky, and M. Ricotti, The Halo Model of Large Scale Structure for Warm Dark Matter, arXiv:1109.6291.

[522] M. Nemevsek, G. Senjanovic, and Y. Zhang, Warm Dark Matter in Low Scale Left-Right Theory, JCAP 1207 (2012) 006, [arXiv:1205.0844].

[523] A. Boyarsky, O. Ruchayskiy, and M. Markevitch, Constraints on parameters of radiatively decaying dark matter from the galaxy cluster 1e0657-56, astro-ph/0611168.

[524] A. Boyarsky, A. Neronov, O. Ruchayskiy, and M. Shaposhnikov, Restrictions on parameters of sterile neutrino dark matter from observations of galaxy clusters, Phys. Rev. D74 (2006) 103506, astro-ph/0603368.

[525] S. Riemer-Sorensen, K. Pedersen, S. H. Hansen, and H. Dahle, Probing the nature of dark matter with cosmic x-rays: Constraints from 'dark blobs' and grating spectra of galaxy clusters, astro-ph/0610034.

[526] H. Yuksel, J. F. Beacom, and C. R. Watson, Strong Upper Limits on Sterile Neutrino Warm Dark Matter, Phys.Rev.Lett. 101 (2008) 121301, [arXiv:0706.4084].

[527] S. Riemer-Sorensen and S. H. Hansen, Decaying dark matter in Draco, arXiv:0901.2569.

[528] A. Boyarsky, O. Ruchayskiy, D. Iakubovskyi, M. G. Walker, S. Riemer-Sorensen, et al., Searching for Dark Matter in X-Rays: How to Check the Dark Matter origin of a spectral feature, Mon.Not.Roy.Astron.Soc. 407 (2010) 1188-1202, [arXiv:1001.0644].

[529] C. R. Watson, Z.-Y. Li, and N. K. Polley, Constraining Sterile Neutrino Warm Dark Matter with Chandra Observations of the Andromeda Galaxy, JCAP 1203 (2012) 018, arXiv:1111.4217. 
[530] H. de Vega and N. Sanchez, Model independent analysis of dark matter points to a particle mass at the keV scale, Mon.Not.Roy.Astron.Soc. 404 (2010) 885, arXiv:0901.0922].

[531] C. Destri, H. de Vega, and N. Sanchez, Fermionic warm dark matter produces galaxy cores in the observed scales because of quantum mechanics, New Astronomy 22, 39 (2013) arXiv:1204.3090.

[532] A. Boyarsky, A. Neronov, O. Ruchayskiy, and I. Tkachev, Universal properties of Dark Matter halos, Phys.Rev.Lett. 104 (2010) 191301, arXiv:0911.3396].

[533] O. R. A. Boyarsky and M. Shaposhnikov, Searching for Dark Matter, Contribution to Open Symposium - European Strategy Preparatory Group, Krakow, Poland, September 2012, https://indico.cern.ch/contributionDisplay.py? contribId=127\& confId=175067 (2012).

[534] A. Boyarsky, D. Iakubovskyi, and O. Ruchayskiy, Next decade of sterile neutrino studies, Phys.Dark Univ. 1 (2012) 136-154, [arXiv:1306.4954].

[535] H. Ishida, K. S. Jeong, and F. Takahashi, Longevity Problem of Sterile Neutrino Dark Matter, arXiv:1309.3069.

[536] L. Bergstrom, The 130 GeV Fingerprint of Right-Handed Neutrino Dark Matter, Phys.Rev. D86 (2012) 103514, [arXiv:1208.6082].

[537] R. Essig, E. Kuflik, S. D. McDermott, T. Volansky, and K. M. Zurek, Constraining Light Dark Matter with Diffuse X-Ray and Gamma-Ray Observations, arXiv:1309.4091.

[538] S. Tremaine and J. E. Gunn, Dynamical role of light neutral leptons in cosmology, Phys. Rev. Lett. 42 (1979) 407-410.

[539] S. Dodelson and L. M. Widrow, Sterile-neutrinos as dark matter, Phys. Rev. Lett. 72 (1994) 17-20, hep-ph/9303287.

[540] T. Asaka, M. Shaposhnikov, and A. Kusenko, Opening a new window for warm dark matter, Phys. Lett. B638 (2006) 401-406, hep-ph/0602150.

[541] F. Bezrukov, H. Hettmansperger, and M. Lindner, keV sterile neutrino Dark Matter in gauge extensions of the Standard Model, Phys.Rev. D81 (2010) 085032, [arXiv:0912.4415].

[542] F. Bezrukov, A. Kartavtsev, and M. Lindner, Leptongenesis in models with keV sterile neutrino dark matter, arXiv:1204.5477.

[543] P. Di Bari, P. Lipari, and M. Lusignoli, The muon-neutrino $\rightarrow$ s-neutrino interpretation of the atmospheric neutrino data and cosmological constraints, Int.J.Mod.Phys. $\mathbf{A 1 5}$ (2000) 2289-2328, hep-ph/9907548.

[544] T. Asaka, M. Laine, and M. Shaposhnikov, Lightest sterile neutrino abundance within the nuMSM, JHEP 0701 (2007) 091, hep-ph/0612182.

[545] V. Klimov, Collective Excitations in a Hot Quark Gluon Plasma, Sov.Phys.JETP 55 (1982) 199-204. 
[546] V. Klimov, Spectrum of Elementary Fermi Excitations in Quark Gluon Plasma. (In Russian), Sov.J.Nucl.Phys. 33 (1981) 934-935.

[547] L. Wolfenstein, Neutrino oscillations in matter, Phys. Rev. D17 (1978) 2369.

[548] S. P. Mikheev and A. Y. Smirnov, Resonance enhancement of oscillations in matter and solar neutrino spectroscopy, Sov. J. Nucl. Phys. 42 (1985) 913-917.

[549] X.-D. Shi and G. M. Fuller, A new dark matter candidate: Non-thermal sterile neutrinos, Phys. Rev. Lett. 82 (1999) 2832-2835, astro-ph/9810076.

[550] S. Das and K. Sigurdson, Cosmological Limits on Hidden Sector Dark Matter, Phys.Rev. D85 (2012) 063510, [arXiv:1012.4458].

[551] R. J. Scherrer and M. S. Turner, Decaying Particles Do Not Heat Up the Universe, Phys.Rev. D31 (1985) 681.

[552] M. Shaposhnikov and I. Tkachev, The nuMSM, inflation, and dark matter, Phys. Lett. B639 (2006) 414-417, hep-ph/0604236.

[553] A. Anisimov, Y. Bartocci, and F. L. Bezrukov, Inflaton mass in the $\nu M S M$ inflation, arXiv:0809.1097.

[554] F. Bezrukov and D. Gorbunov, Light inflaton Hunter's Guide, JHEP 1005 (2010) 010, arXiv:0912.0390.

[555] F. Bezrukov, D. Gorbunov, and M. Shaposhnikov, On initial conditions for the Hot Big Bang, JCAP 0906 (2009) 029, arXiv:0812.3622].

[556] K. Petraki and A. Kusenko, Dark-matter sterile neutrinos in models with a gauge singlet in the Higgs sector, Phys.Rev. D77 (2008) 065014, arXiv:0711.4646.

[557] D. Gorbunov and A. Panin, Scalaron the mighty: producing dark matter and baryon asymmetry at reheating, Phys.Lett. B700 (2011) 157-162, arXiv:1009.2448.

[558] J. Bond, G. Efstathiou, and J. Silk, Massive Neutrinos and the Large Scale Structure of the Universe, Phys.Rev.Lett. 45 (1980) 1980-1984.

[559] A. J. Benson, A. Farahi, S. Cole, L. A. Moustakas, A. Jenkins, et al., Dark Matter Halo Merger Histories Beyond Cold Dark Matter: I - Methods and Application to Warm Dark Matter, arXiv:1209.3018.

[560] J. Sommer-Larsen, P. Naselsky, I. Novikov, and M. Gotz, Inhomogenous primordial baryon distributions on sub-Galactic scales: High-z Galaxy formation with WDM, Mon.Not.Roy.Astron.Soc. 352 (2004) 299, astro-ph/0309329.

[561] B. W. O'Shea and M. L. Norman, Population III star formation in a lambda-wdm universe, Astrophys.J. 648 (2006) 31-46, astro-ph/0602319.

[562] P. Bode, J. P. Ostriker, and N. Turok, Halo formation in warm dark matter models, Astrophys. J. 556 (2001) 93-107, astro-ph/0010389. 
[563] S. H. Hansen, J. Lesgourgues, S. Pastor, and J. Silk, Constraining the window on sterile neutrinos as warm dark matter, Mon. Not. Roy. Astron. Soc. 333 (2002) 544-546, astro-ph/0106108.

[564] M. Viel, J. Lesgourgues, M. G. Haehnelt, S. Matarrese, and A. Riotto, Constraining warm dark matter candidates including sterile neutrinos and light gravitinos with wmap and the lyman-alpha forest, Phys. Rev. D71 (2005), no. 6 063534, astro-ph/0501562.

[565] M. Viel, G. D. Becker, J. S. Bolton, M. G. Haehnelt, M. Rauch, et al., How cold is cold dark matter? Small scales constraints from the flux power spectrum of the high-redshift Lyman-alpha forest, Phys.Rev.Lett. 100 (2008) 041304, [arXiv:0709.0131].

[566] R. K. de Naray, G. D. Martinez, J. S. Bullock, and M. Kaplinghat, The Case Against Warm or Self-Interacting Dark Matter as Explanations for Cores in Low Surface Brightness Galaxies, arXiv:0912.3518.

[567] F. Villaescusa-Navarro and N. Dalal, Cores and Cusps in Warm Dark Matter Halos, JCAP 1103 (2011) 024, arXiv:1010.3008.

[568] A. Schneider, R. E. Smith, A. V. Maccio, and B. Moore, Nonlinear Evolution of Cosmological Structures in Warm Dark Matter Models, arXiv:1112.0330.

[569] C. Power, Seeking Observable Imprints of Small-Scale Structure on the Properties of Dark Matter Haloes, arXiv:1309.1591.

[570] V. Lora, A. Just, F. Sanchez-Salcedo, and E. Grebel, Dark Matter Subhalos in the Ursa Minor Dwarf Galaxy, Astrophys.J. 757 (2012) 87, [arXiv:1207.5681].

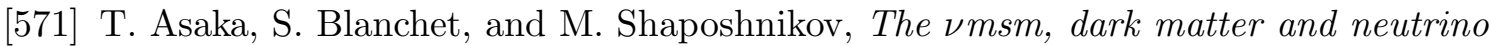
masses, Phys. Lett. B631 (2005) 151-156, hep-ph/0503065.

[572] T. Asaka and M. Shaposhnikov, The $\nu m s m$, dark matter and baryon asymmetry of the universe, Phys. Lett. B620 (2005) 17-26, hep-ph/0505013.

[573] M. Shaposhnikov, Is there a new physics between electroweak and planck scales?, hep-th/0708.3550 (2007) [arXiv:0708.3550].

[574] M. Shaposhnikov and D. Zenhausern, Scale invariance, unimodular gravity and dark energy, Phys. Lett. B671 (2009) 187-192, [arXiv:0809.3395].

[575] M. Shaposhnikov and D. Zenhausern, Quantum scale invariance, cosmological constant and hierarchy problem, Phys. Lett. B671 (2009) 162-166, [arXiv:0809.3406].

[576] N. Sahu and U. A. Yajnik, Dark matter and leptogenesis in gauged B - L symmetric models embedding nu MSM, Phys.Lett. B635 (2006) 11-16, hep-ph/0509285.

[577] A. Boyarsky, A. Neronov, O. Ruchayskiy, and M. Shaposhnikov, The masses of active neutrinos in the $\nu$ msm from x-ray astronomy, JETP Lett. 83 (2006) 133-135, hep-ph/0601098.

[578] D. Gorbunov and M. Shaposhnikov, Kaon physics within nuMSM, PoS KAON (2008) 047. 
[579] F. Bezrukov, nuMSM and its experimental tests, J.Phys.Conf.Ser. 110 (2008) 082002, arXiv:0710.2501.

[580] F. L. Bezrukov and M. Shaposhnikov, The Standard Model Higgs boson as the inflaton, Phys. Lett. B659 (2008) 703-706, arXiv:0710.3755.

[581] J. Garcia-Bellido, D. G. Figueroa, and J. Rubio, Preheating in the Standard Model with the Higgs-Inflaton coupled to gravity, Phys. Rev. D79 (2009) 063531, arXiv:0812.4624.

[582] M. Shaposhnikov, The $\nu M S M$, leptonic asymmetries, and properties of singlet fermions, JHEP 08 (2008) 008, arXiv:0804.4542.

[583] V. Gorkavenko and S. Vilchynskiy, Some constraints on the Yukawa parameters in the neutrino modification of the Standard Model (nuMSM) and CP-violation, Eur.Phys.J. C70 (2010) 1091-1098, [arXiv:0907.4484].

[584] T. Asaka and H. Ishida, Flavour Mixing of Neutrinos and Baryon Asymmetry of the Universe, Phys.Lett. B692 (2010) 105-113, arXiv:1004.5491.

[585] J. Garcia-Bellido, J. Rubio, M. Shaposhnikov, and D. Zenhausern, Higgs-Dilaton Cosmology: From the Early to the Late Universe, Phys.Rev. D84 (2011) 123504, arXiv:1107.2163].

[586] F. Bezrukov, D. Gorbunov, and M. Shaposhnikov, Late and early time phenomenology of Higgs-dependent cutoff, JCAP 1110 (2011) 001, arXiv:1106.5019.

[587] V. M. Gorkavenko, I. Rudenok, and S. I. Vilchynskiy, Leptonic asymmetry of the sterile neutrino hadronic decays in the $\nu M S M$, arXiv:1201.0003.

[588] K. Allison, Dark matter, singlet extensions of the nuMSM, and symmetries, arXiv:1210.6852.

[589] L. Canetti and M. Shaposhnikov, The $\nu$ MSM and muon to electron conversion experiments, Hyperfine Interact. 214 (2013), no. 1-3 5-11.

[590] A. Boyarsky, J. Frohlich, and O. Ruchayskiy, Self-consistent evolution of magnetic fields and chiral asymmetry in the early Universe, Phys.Rev.Lett. 108 (2012) 031301, [arXiv:1109.3350].

[591] P. A. Dirac, The Quantum theory of electron, Proc.Roy.Soc.Lond. A117 (1928) 610-624.

[592] P. B. Pal, Dirac, Majorana and Weyl fermions, Am. J. Phys. 79 (2011) 485-498, [arXiv:1006.1718].

[593] E. Majorana, Theory of the Symmetry of Electrons and Positrons, Nuovo Cim. 14 (1937) $171-184$. 